\title{
Automorphisms and isomorphisms of some $p$-ary bent functions
}

\section{Ulrich Dempwolff ${ }^{1}$}

Received: 30 October 2018 / Accepted: 22 March 2019 / Published online: 23 May 2019

(c) Springer Science+Business Media, LLC, part of Springer Nature 2019

\begin{abstract}
In the predecessor to this paper Dempwolff (Comm Algebra 34(3):1077-1131, 2006), group-theoretic methods were used to solve automorphism and equivalence questions for (certain) ordinary bent functions, i.e., bent functions over $\mathbb{F}_{2}$. Here, we consider the same problems for $p$-ary bent functions, $p$ an odd prime and solve these questions for functions analogous to those which appear in Dempwolff (Comm Algebra 34(3): 10771131, 2006). Although our general analysis is similar to the approach of Dempwolff (Comm Algebra 34(3):1077-1131, 2006), it turns out that the odd characteristic leads to simplifications: Often, the double derivative can be computed (cf. Lemma 2.10) and factorizations of the automorphism group (cf. Lemma 2.3) can be established resulting in restrictions for automorphisms and equivalence maps.
\end{abstract}

Keywords Bent function · Automorphism group · EA equivalence · Cohomology

\section{Introduction}

Equivalence and automorphism problems of bent functions are known to be difficult. Since the number of bent functions grows exponentially with the number of variables, simple invariants like the algebraic degree will usually not suffice to solve equivalence questions. So stronger methods seem to be necessary. In [9], the author considered classes of ordinary bent functions (bent functions over $\mathbb{F}_{2}$ ) for which such questions can be successfully treated with group-theoretic methods. For these examples, group theory works, since large groups of automorphisms are visible due to the special nature of the examples.

In this article, we consider the analogous $p$-ary bent functions for primes $p>2$. Now the analysis becomes easier, since the invariant "double derivative" (cf. Sect. 2.3)

$凶$ Ulrich Dempwolff

dempwolff@mathematik.uni-kl.de

1 Department of Mathematics, University of Kaiserslautern, Erwin-Schroedinger-Strasse, 67653

Kaiserslautern, Germany 
can be computed. Moreover, the odd characteristic gives rise to a factorization lemma (Lemma 2.3), which allows to reduce the problem of EA equivalence to the more restricted problem of linear equivalence (see Cor. 2.4).

In the next section, we review preparatory results mainly from group theory. These facts are basic, but an exception is Sect. 2.5 on the $\mathrm{H}^{1}$-cohomology of symplectic groups, where we collect details needed for the specific Example 3.8.

In Sect. 3, we introduce the examples, which will be investigated in the subsequent sections with regard to automorphisms and equivalence. This section starts with a general construction of bent function. In Examples 3.4, 3.6 and 3.8, we consider special cases of this construction, cases in which large groups of automorphisms are visible by virtue of the construction. Example 3.13 introduces a very simple type of Maiorana functions, again admitting large groups of automorphisms.

In Sect. 4, Theorem 4.1 and Corollaries 4.2 and 4.3 solve the automorphism and equivalence problem of the examples from 3.4, 3.6 and 3.8. Theorem 5.1 and Corollary 5.2 of Sect. 5 handle these questions for bent functions from Example 3.13.

Our proofs are mainly of group-theoretic nature, but (as already mentioned) we require only basic knowledge of group theory and a few facts from cohomology theory. Standard texts like $[1,16]$ cover the needed background. Our notation follows the conventions of group theory; in particular, we write the symbol of an homomorphism on the right-hand side of the argument.

\section{Preliminaries}

\subsection{Bent functions}

Let $p$ be a prime, $V=V(n, p)$ an $n$-dimensional $\mathbb{F}_{p}$-space and $(\cdot, \cdot): V \times V \rightarrow \mathbb{F}_{p}$ a non-degenerate symmetric bilinear form. The function $f: V \rightarrow \mathbb{F}_{p}$ is a p-ary bent function or short a bent function if for all $v \in V$

$$
\text { (B1) } \widehat{f}(v) \widehat{\widehat{f}(v)}=p^{n}
$$

where

$$
\widehat{f}(v)=\sum_{x \in V} \zeta_{p}^{f(x)-(x, v)}
$$

is the Walch coefficient at $v$ and $\zeta_{p}=\exp (2 \pi i / p)$. For a function $f: V \rightarrow \mathbb{F}_{p}$, define the complex $p^{n} \times p^{n}$-matrix $H_{f}$ by

$$
H_{f}(x, y)=\zeta_{p}^{f(x-y)}, \quad x, y \in V .
$$

Then, $f$ is bent (cf. [19, Prop. 4]) iff:

$$
H_{f} H_{f}^{*}=p^{n} \mathbf{1}_{p^{n} \times p^{n}}
$$


where $H_{f}^{*}$ is the complex matrix, whose entries are obtained by complex conjugation and transposition of $H_{f}$.

Remark 2.1 (a) We use criterion (B2) in the form $\sum_{x \in V} \zeta_{p}^{f(x)-f(x+b)}=0$ for all $0 \neq b \in V$.

(b) For $p=2$, bent functions on $V(n, 2)$ only exist if $n$ is even, while for $p>2$ bent functions exist in any dimension. A quadratic form $Q$ on $V(n, p)$ is bent, iff the associated bilinear form (obtained by polarization) is non-degenerate.

\subsection{Equivalence and automorphisms of functions}

Let $F$ be a field and $V$ be an $F$-space. For $v \in V$, we define the translation $\tau_{v}$ : $V \rightarrow V$ with $v$ by $\tau_{v}: x \mapsto x+v$. An affine transformation $\alpha$ on $V$ has the form $\alpha=\Phi \circ \tau_{v}, \Phi \in \operatorname{End}(V)$. We denote by $\operatorname{AGL}(V)$ the group of invertible affine transformation. Clearly, AGL $(V)$ is the semidirect product of $\tau(V)$ with $\operatorname{GL}(V)$ : $\operatorname{AGL}(V)=\operatorname{GL}(V) \tau(V)$. If $V=V_{1} \oplus V_{2}$ is a decomposition and $\Phi \in \operatorname{GL}(V)$ fixes $V_{1}$ and $V_{2}$, we write $\Phi=\operatorname{diag}\left(\Phi_{1}, \Phi_{2}\right)$ where $\Phi_{i}=\Phi_{V_{i}}$. More generally, we write $\Phi=\left(\begin{array}{ll}\Phi_{11} & \Phi_{12} \\ \Phi_{21} & \Phi_{22}\end{array}\right)$ with $\Phi_{i j} \in \operatorname{Hom}_{F}\left(V_{i}, V_{j}\right)$, such that for $v_{i} \in V_{i}$ we have $\left(v_{1}+v_{2}\right) \Phi=v_{1} \Phi_{11}+v_{2} \Phi_{21}+v_{1} \Phi_{12}+v_{2} \Phi_{22}$. Let $(\cdot, \cdot): V \times V \rightarrow F$ be a nondegenerate symmetric bilinear form on $V$ and $W, U$ be subspaces, which are dual with respect to $(\cdot, \cdot)$. For $\Phi \in \operatorname{End}(U)$, we denote by $\Phi^{*} \in \operatorname{End}(W)$ the adjoint operator, i.e., $(u \Phi, w)=\left(u, w \Phi^{*}\right)$ for all $u \in U, w \in W$. Similarly, if $\Phi \in \operatorname{Hom}(U, W)$, then $\Phi^{*} \in \operatorname{Hom}(U, W)$ is the unique operator with $\left(u_{1} \Phi, u_{2}\right)=\left(u_{1}, u_{2} \Phi^{*}\right)$ for all $u_{1}, u_{2} \in U$.

Let $V, W$ be $F$-spaces and $f: V \rightarrow W$ a function. We call $\mathcal{U}=\mathcal{U}(f)=V \times W$ the ambient space of $F$, and $\Gamma(f)=\{(x, f(x)) \mid x \in V\} \subseteq \mathcal{U}$ is the graph of $f$. Let $g: V \rightarrow W$ be another function. We call $f$ and $g C C Z$ equivalent, if there exists $\alpha \in \operatorname{AGL}(\mathcal{U})$ with $\Gamma(g)=\Gamma(f) \alpha$. A CCZ isomorphism $\alpha=\Phi \circ \tau_{u}, \Phi \in \operatorname{GL}(\mathcal{U})$, $u \in \mathcal{U}$ is an EA isomorphism (EA = extended affine) if $W$ is invariant under $\Phi$, and $\alpha$ is an affine isomorphism if $V$ is invariant under $\Phi$ too. For our purposes, it is convenient to call an affine isomorphism linear if its translation part is trivial (i.e., $u=0$ ). The groups of EA automorphisms and affine automorphisms are denoted by $\mathbf{E A}(f)$ and $\mathbf{A}(f)$, respectively.

Alternatively, an element $\alpha \in \mathbf{E A}(f)$ can be uniquely described by $\Phi_{11} \in \operatorname{GL}(V)$, $\Phi_{22} \in \mathrm{GL}(W), v \in V, w \in W$, and $\Phi_{12} \in \operatorname{Hom}(V, W)$ such that for all $x \in V$ the equation

$$
f\left(x \Phi_{11}+v\right)=f(x) \Phi_{22}+x \Phi_{12}+w
$$

holds; $\alpha$ lies in $\mathbf{A}(f)$ if $\Phi_{12}=0$.

Our investigation of bent functions will only concern EA equivalence, since $\mathbf{C C Z}$ equivalent bent functions are already EA equivalent (cf. [4]).

We denote by $\mathbf{E}(f)$ the subgroup of elements in $\mathbf{E A}(f)$, whose linear parts act trivially on $W$ and $\mathcal{U} / W$. Clearly, $\mathbf{E}(f)$ is a normal subgroup of $\mathbf{E A}(f)$. If $\Phi \circ \tau_{u} \in$ 
$\mathbf{E}(f), \Phi \in \operatorname{GL}(\mathcal{U}), u \in \mathcal{U}$, then $(x, z) \Phi=\left(x, z+x \Phi_{12}\right)$ with $\Phi_{12} \in \operatorname{Hom}(V, W)$. With this notation, we have:

Lemma 2.2 Let $p>0$ be the characteristic of the finite vector space $V$.

(a) $\mathbf{E}(f)$ is an elementary abelian p-group.

(b) The mapping $\mathbf{E}(f) \ni \Phi \circ \tau_{u} \mapsto u+(0 \times W) \in \mathcal{U} /(0 \times W)$ is a monomorphism.

(c) Let $f$ be bent and $\Phi \circ \tau_{u} \in \mathbf{E}(f)$. Then, $\Phi \circ \tau_{u}=1$ iff $\Phi=1$.

Proof Assertion (a) is a consequence of (b).

For (b), we observe that the mapping is a homomorphism and $\alpha=\Phi \circ \tau_{u}$ lies in the kernel of this mapping iff $u=(0, c) \in 0 \times W$. Then, $\alpha: \Gamma(f) \ni(x, f(x)) \mapsto$ $\left(x, f(x)+x \Phi_{12}+c\right) \in \Gamma(f)$. This shows $f(x)=f(x)+x \Phi_{12}+c$, therefore $0=x \Phi_{12}+c$ for all $x$. Then, $c=0$ and $\Phi_{12}=0$, i.e., $\Phi \circ \tau_{u}=1$.

To (c): Finally, if $\tau_{u} \in \mathbf{E}(f)$, then $f(x+v)=f(x)+c$ for $u=(v, c)$. By Remark 2.1(a) first $v=0$ and then $c=0$, i.e., $u=0$.

Under mild assumptions, we have the following factorization of $\mathbf{E A}(f)$.

Lemma 2.3 Let $p>2$ be a prime, $V, W$ finite-dimensional $\mathbb{F}_{p}$-spaces and $f: V \rightarrow$ $W$, a function with $f(0)=0$ and $f(-x)=f(x)$ for all $x \in V$. Set $\mathcal{U}=\mathcal{U}(f)$. Then, $\mathbf{E A}(f)=(\mathbf{A}(f) \cap \mathrm{GL}(\mathcal{U})) \mathbf{E}(f)$ and $\mathbf{A}(f) \leq \mathrm{GL}(\mathcal{U})$, if $f$ is bent.

Proof We will prove this lemma by an Frattini argument. By assumption, $\mathbf{A}(f)$ contains the element $\iota=\operatorname{diag}\left(-1_{V}, 1_{W}\right)$. A quick computation shows $\alpha^{-1} \iota \alpha \equiv \iota$ $(\bmod \mathbf{E}(f))$ for $\alpha \in \mathbf{E A}(f)$. So $\langle\iota, \mathbf{E}(f)\rangle \unlhd \mathbf{E A}(f)$ and $\langle\iota \mathbf{E}(f)\rangle \leq Z(\mathbf{E A}(f) / \mathbf{E}(f))$. Let $g \in \mathbf{E A}(f)$. Then, $\left\langle\iota^{g}\right\rangle \in \operatorname{Syl}_{2}(\langle\iota, \mathbf{E}(f)\rangle)$ by Lemma 2.2. Therefore, there exists $h \in\langle\iota, \mathbf{E}(f)\rangle$ with $\left\langle\iota^{g h}\right\rangle=\langle\iota\rangle$. This shows $g h \in C_{\mathbf{E A}(f)}(\iota)$, and we conclude $\mathbf{E A}(f)=C_{\mathbf{E A}(f)}(\iota) \mathbf{E}(f)$.

We claim $\mathbf{A}(f) \cap \operatorname{GL}(\mathcal{U})=C_{\mathbf{E A}(f)}(\iota)$ : Clearly, $\mathbf{A}(f) \cap \operatorname{GL}(\mathcal{U}) \leq C_{\mathbf{E A}(f)}(\iota)$. Let $\alpha=\Phi \circ \tau_{u} \in C_{\mathbf{E A}(f)}(\iota)$. We have $\Phi^{-1} \Phi^{\iota}=\tau_{u}\left(\tau_{-u}\right)^{\iota} \in \mathrm{GL}(\mathcal{U}) \cap \tau(U)=1$ forcing $\Phi \in C_{\mathrm{GL}(\mathcal{U})}(\iota)$ and $u \in 0 \times W=C_{\mathcal{U}}(\iota)$. Also $\Phi$ leaves invariant the decomposition $\mathcal{U}=V \oplus W=[\mathcal{U}, \iota] \oplus C_{\mathcal{U}}(\iota)$. As $(0,0) \in \Gamma(f)$, we finally obtain $u=0$. Hence, $\mathbf{A}(f) \cap \mathrm{GL}(\mathcal{U})=C_{\mathbf{E A}(f)}(\iota)$.

Let $f$ be bent and $\alpha=\Phi \circ \beta \in \mathbf{A}(f)$ with $\Phi \in \mathbf{A}(f) \cap \operatorname{GL}(\mathcal{U})$ and $\beta \in \mathbf{E}(f)$. The linear parts of $\alpha$ and $\Phi$ are fixing $V$. Thus, the linear part of $\beta$ fixes $V$ too, i.e., we have $\beta=\tau_{u}$ for some $u \in \mathcal{U}$ and $u=0$ by Lemma 2.2(c), i.e., $\mathbf{A}(f) \leq \operatorname{GL}(\mathcal{U})$.

A consequence of this lemma is a simplified criterion for EA equivalence:

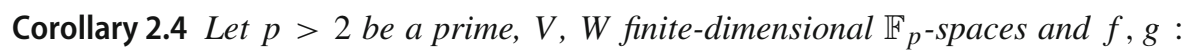
$V \rightarrow W$, functions with $f(0)=g(0)=0$ and $f(-x)=f(x), g(-x)=g(x)$ for all $x \in V$. Let $f$ and $g$ be EA equivalent. Then, they are linear equivalent.

Proof We know that $\iota \in \mathbf{A}(f) \cap \mathbf{A}(g)$, where $\iota$ is defined as in the proof of Lemma 2.3. Let $\psi \in \operatorname{AGL}(\mathcal{U})$, such that the linear part of $\psi$ fixes $W$ and that $\Gamma(g)=\Gamma(f) \psi$. Then, $\mathbf{E A}(g)=\psi^{-1} \mathbf{E A}(f) \psi, \mathbf{E}(g)=\psi^{-1} \mathbf{E}(f) \psi$, and $\iota^{\psi} \in \mathbf{E}(g)\langle\iota\rangle$. We can adjust $\psi$ by some element in $\mathbf{E}(g)\langle\iota\rangle$, such that $\psi \in C_{\mathrm{AGL}(\mathcal{U})}(\iota)$. We have seen in the proof of Lemma 2.3 that $\psi=\Phi \circ \tau_{w}$, where $\Phi \in \operatorname{GL}(\mathcal{U})$ fixes $V \times 0$ and $0 \times W$ and $w \in W$. Since $\Gamma(g)=\Gamma(f) \psi$ and as $f(0)=g(0)=0$, we have $w=0$. The proof is complete. 
Remark 2.5 Let $p$ be an odd prime $V, W$ finite-dimensional $\mathbb{F}_{p}$-spaces and $f: V \rightarrow$ $W$ a function satisfying the assumptions of Lemma 2.3. In particular, if $G=\mathbf{A}(f) \cap$ $\operatorname{GL}(\mathcal{U})$ and $\mathcal{U}=V \times W$, then $G$ leaves the spaces $V \times 0$ and $0 \times W$ invariant. An element of $\mathbf{E}(f)$ is denoted by $\beta(v)$, with a unique $v \in V$ (Lemma 2.2), when

$$
(x, z) \beta(v)=\left(x+v, x \lambda_{v}+g(v)+z\right), \quad(x, z) \in \mathcal{U},
$$

with $\lambda_{v} \in \operatorname{Hom}(V, W)$ and $g(v) \in W$. In fact, as $(v, g(v))=(0,0) \beta(v) \in \Gamma(f)$, we obtain

$$
g(v)=f(v) .
$$

As $\beta(u+v)=\beta(u) \circ \beta(v)$, there exists a subspace $V_{0} \subseteq V$, such that $\mathbf{E}(f)=\beta\left(V_{0}\right)$. Moreover, $\lambda: V_{0} \rightarrow \operatorname{Hom}(V, W)$ is a homomorphism and we have

$$
x \lambda_{v}=f(x+v)-f(x)-f(v),
$$

since $(x+v, f(x+v))=(x, f(x)) \beta(v)=\left(x+v, x \lambda_{v}+f(v)+f(x)\right)$. In particular, if $W=\mathbb{F}_{p}$, then the restriction of $f$ to $V_{0}$ is a quadratic form. Acting with $G$ on $\mathbf{E}(f)$ by conjugation, we see

$$
\Phi^{-1} \beta(v) \Phi=\beta(v \Phi) .
$$

Example 2.6 Let $Q$ be a non-degenerate quadratic form on $V=V(n, p)$ and denote by $(\cdot, \cdot)$ the (non-degenerate) bilinear form obtained from polarization of $Q$. An element $\Phi \in \mathrm{GL}(V)$ is called a similarity, if there exists $r=r_{\Phi} \in \mathbb{F}_{p}^{*}$, such that $Q(x \Phi)=$ $r Q(x)$ for all $x \in V$. One calls $r_{\Phi}$ the multiplier of $\Phi$, and we denote by $\operatorname{GO}(V, Q)$ the group of similarities. If $r_{\Phi}=1$ one calls $\Phi$ an isometry; the group of isometries $\mathrm{O}(V, Q)$ is the orthogonal group (on $V)$. Clearly, the group

$$
H=\left\{\operatorname{diag}\left(\Phi, r_{\Phi}\right) \in \mathrm{GL}(\mathcal{U}) \mid \Phi \in \mathrm{GO}(V, Q)\right\} \simeq \mathrm{GO}(V, Q),
$$

$\mathcal{U}=V \times \mathbb{F}_{p}$ is a subgroup of $\mathbf{A}(Q)$. Assume now $p=2$, and let $0 \neq v \in V$. Define a symplectic transvection $t(v) \in \mathrm{GL}(V)$ by $x t(v)=x+(v, x) v$. Then, $t(v) \in \mathrm{O}(V, Q)$ if $Q(v)=1$. If, however, $v$ is singular (i.e., $Q(v)=0$ ), then $t(v)$ lies in the symplectic group $\operatorname{Sp}(V)=\{\Phi \in \mathrm{GL}(V) \mid(x \Phi, y \Phi)=(x, y), x, y \in V\}$ but not in $\operatorname{GO}(V, Q)$. We extend $t(v)$ to $\mathcal{U}$ by $(x, z) t(v)=(x+(x, v) v, z)$ and if $v$ is singular we set $\alpha(v)=t(v) \circ \tau_{v} \in \operatorname{AGL}(\mathcal{U})$. We observe $\alpha(v) \in \mathbf{A}(Q)$ and that $\operatorname{Sp}(V) \simeq\langle H, \alpha(v)| 0 \neq v \in V$ singular $\rangle \leq \mathbf{A}(Q)$.

By Lemma 2.2 and Remark 2.5, $\mathbf{E}(Q)=\beta(V) \simeq(V,+)$. Here, $\beta(v)=\lambda_{v}$ 。 $\tau_{(v, Q(v))}$ with $(x, z) \lambda_{v}=(x,(x, v)+z)$. Hence, for $p>2$ the group $\mathbf{E A}(V)$ contains a subgroup isomorphic to $\operatorname{GO}(V, Q) V$ (semidirect product), while for $p=2$ the group $\mathbf{E A}(V)$ contains a subgroup isomorphic to $\operatorname{Sp}(V) V$ (semidirect product).

Remark 2.7 (a) The classification of non-degenerate quadratic forms over prime field $\mathbb{F}_{p}$ distinguishes the following types [14, Thm. 12.9]: 
(1) $n=2 m, Q^{+}(X)=\sum_{i=1}^{m} X_{i} X_{n+1-i}$ (hyperbolic type).

(2) $n=2 m, Q^{-}(X)=\sum_{i=1}^{m-1} X_{i} X_{n+1-i}+X_{m}^{2}+a X_{m} X_{m+1}+b X_{m+1}^{2}$ (elliptic type). Here, $X^{2}+a X+b$ is an irreducible polynomial over $\mathbb{F}_{p}$.

(3) $n=2 m+1, p>2, Q(X)=\sum_{i=1}^{m} X_{i} X_{n+1-i}+X_{m+1}^{2}$.

Assume $p=2$. Then, $\left(X_{m}+1\right)\left(X_{m+1}+1\right)=X_{m}^{2}+X_{m} X_{m+1}+X_{m+1}^{2}+1$ (as $X_{i}^{2} \equiv X_{i}$ as a function over $\mathbb{F}_{2}$ ), which shows that $Q^{+}$and $Q^{-}$are affine equivalent as bent functions.

(b) Let $p>2$. The mapping $\operatorname{GO}(V, Q) \ni \Phi \mapsto r_{\Phi} \in \mathbb{F}_{p}$ of similarities on their multipliers is a homomorphism, whose image is $\mathbb{F}_{p}^{*}$ if $n$ is even and $\left(\mathbb{F}_{p}^{*}\right)^{2}$ if $n$ is odd (cf. [15, Prop. 5(b)]).

(c) Let $(V, Q)$ be of hyperbolic type, $V=W \oplus W^{\prime}$ a decomposition into totally singular spaces. Let $r$ be a generator of $\mathbb{F}_{p}^{*}$. Then, $\operatorname{diag}\left(1_{W}, r 1_{W^{\prime}}\right)$ is a similarity with multiplier $r$.

Proposition 2.8 Let $p$ be a prime and let $Q$ be a non-degenerate quadratic form on $V=V(n, p)$.

(a)

$$
\mathbf{A}(Q) \simeq \begin{cases}\mathrm{GO}(V, Q), & p>2 \\ \operatorname{Sp}(V), & p=2,\end{cases}
$$

where $n$ is even for $p=2$ and $\operatorname{Sp}(V)$ is the symplectic group with respect to the bilinear form obtained by polarization of $Q$.

(b) $\mathbf{E A}(Q) \simeq \mathbf{A}(Q) \mathbf{E}(Q), \mathbf{E}(Q)=\beta(V) \simeq(V,+)$.

Proof Let $(\cdot, \cdot)$ be the bilinear form obtained by polarization from $Q$ and $\mathcal{U}=V \times \mathbb{F}_{p}$ the ambient space of $Q$.

Let $\alpha=\Phi \circ \tau_{u}$ be an element in $\mathbf{E A}(Q)$ with $\Phi \in \operatorname{GL}(\mathcal{U})$, and $u=(v, b) \in \mathcal{U}$. Assume $(x, z) \Phi=\left(x \Phi_{1}, x L_{w}+a z\right)$ with $x L_{v}=(x, v), \Phi_{1} \in \operatorname{GL}(V), w \in V$, and $a \in \mathbb{F}_{p}^{*}$, i.e., $Q\left(x \Phi_{1}+v\right)=a Q(x)+x L_{w}+b$ for all $x$. Then, $Q(v)=b(\operatorname{set} x=0)$, so that $Q\left(x \Phi_{1}\right)+\left(x \Phi_{1}, v\right)=a Q(x)+x L_{w}$. Replace $x$ by $x+y$ and we get

$$
\left(x \Phi_{1}, y \Phi_{1}\right)=a(x, y)
$$

for all $x, y$. So $\Phi_{1}$ is a similarity with respect to $(\cdot, \cdot)$, i.e., $\Phi_{1} \in \mathrm{GO}(V, Q)$ if $p>2$ and $\Phi_{1} \in \operatorname{Sp}(V)$ if $p=2$. But we know by Example 2.6 that there exists $H \leq \mathbf{A}(Q) \cap$ $\mathrm{GL}(\mathcal{U})$, such that $H \simeq \mathrm{GO}(V, Q)$ if $p>2$ and $H \simeq \mathrm{Sp}(V)$ if $p=2$. So adjusting $\alpha$ with an element from $H$, we may assume $\Phi_{1}=1$. So $Q(x+v)=a Q(x)+x L_{w}+b$ for all $x$, which leads to $\alpha=\beta(v)$. By Example 2.6, (a) and (b) follow.

Remark 2.9 (a) Let $f$ and $g$ be two EA equivalent functions, then $\mathbf{E A}(f) \simeq \mathbf{E A}(g)$ : Namely, if $\Psi: f \rightarrow g$ is an EA equivalence map, then $\mathbf{E A}(g)=\Psi^{-1} \mathbf{E A}(f) \Psi$.

(b) Let $n$ be even and $Q^{+}$and $Q^{-}$be the two non-degenerate quadratic forms from Remark 2.7 on $V=V(n, p)$. We have already seen that the two forms are affine equivalent for $p=2$. For $p>2$, however, $\left|\mathrm{GO}\left(V, Q^{+}\right)\right| \neq\left|\mathrm{GO}\left(V, Q^{-}\right)\right|$(cf. [16, Bem. II,10.16]), so by Proposition $2.8 Q^{+}$and $Q^{-}$are not EA equivalent if $p>2$. 
(c) If $p>2$, then $H=\mathbf{A}(Q) \leq \mathrm{GL}(U)$ leaves invariant the decomposition $\mathcal{U}=$ $V \oplus \mathcal{U}_{0}$ in accordance with Lemma 2.3. For $p=2$, however, the assertions of this lemma do not hold: Here, $H_{0}=\mathbf{A}(Q) \simeq \operatorname{Sp}(V)$ lies in $\operatorname{AGL}(\mathcal{U})$, but not in $\operatorname{GL}(\mathcal{U})$. On the other hand, $\operatorname{Sp}(V) \simeq H_{1}=\langle H, \alpha(v) \circ \beta(v)| 0 \neq v \in V$ singular $\rangle$ lies in $\operatorname{GL}(\mathcal{U})$ but acts indecomposably on $\mathcal{U}$, i.e., $\mathcal{U}_{0}$ is the socle of the $\mathbb{F}_{2} H_{1}$-module $\mathcal{U}$. Note that $H_{0}$ and $H_{1}$ represent the two conjugacy classes of complements of $\mathbf{E}(Q)$ in $\mathbf{E A}(Q)$ (it is well known that $\left.\mathrm{H}^{1}(\operatorname{Sp}(2 n, 2), V(2 n, 2)) \simeq \mathbb{F}_{2}\right)$.

\subsection{Derivatives}

Let $V, W$ be two $F$-spaces and $f: V \rightarrow W$ a function. Let $v \in V$. We call the function $D_{v} f: V \rightarrow W$ defined by $D_{v} f(x)=f(x+v)-f(x)$ the derivative of $f$ in direction of $v$. We need the following properties.

Lemma 2.10 (a) For all $x \in V$, we have $D_{v} D_{v} f(x)=D_{2 v} f(x)-2 D_{v} f(x)$.

(b) Let $v, a \in V, b \in W$ and $\Phi_{11} \in \operatorname{End}(V)$, $\Phi_{22} \in \operatorname{End}(W)$ and $\Phi_{12} \in \operatorname{Hom}(V, W)$. Set $g(x)=f\left(x \Phi_{11}+a\right) \Phi_{22}+x \Phi_{12}+b$. Then

$$
D_{v} g(x)=D_{v \Phi_{11}} f\left(x \Phi_{11}+a\right) \Phi_{22}+v \Phi_{12}
$$

Proof (a)

$$
\begin{aligned}
D_{v} D_{v} f(x) & =D_{v}(f(x+v)-f(x)) \\
& =f(x+2 v)-f(x+v))-(f(x+v)-f(x)) \\
& =(f(x+2 v)-f(x))-2(f(x+v)-f(x)) \\
& =D_{2 v} f(x)-2 D_{v} f(x)
\end{aligned}
$$

(b) follows by a similar routine calculation.

Remark 2.11 Let $p>2$ be a prime, $f: V=V(n, p) \rightarrow \mathbb{F}_{p}$ a function and $\alpha \in$ $\mathbf{E A}(f)$. Then, there exist $\Phi \in \mathrm{GL}(V), a \in V, L \in \operatorname{Hom}\left(V, \mathbb{F}_{p}\right)$ and $b, c \in \mathbb{F}_{p}$, $c \neq 0$, such that $\alpha=\Phi^{*} \tau_{(a, b)}$, with $\Phi^{*}: \mathcal{U}=V \times \mathbb{F}_{p} \ni(x, z) \mapsto(x \Phi, z c+x L)$, i.e., $f(x \Phi+a)=f(x) c+x L+b$ for all $x \in V$. By Lemma 2.10

$$
D_{v \Phi}^{2} f(x \Phi+a)=D_{v}^{2} f(x) c
$$

for all $x \in V$. Therefore, $\Phi$ leaves invariant the set $\left\{v \in V \mid D_{v}^{2} f=0\right\}$ of vectors with a trivial double derivative.

\subsection{Representations of cyclic groups}

Let $q$ be a prime power. An operator $\sigma \in \operatorname{GL}(n, q)$ of order $q^{n}-1$ is called a Singer cycle, and we call the group generated by $\sigma$ a Singer group. The following result is well known; see, for instance, [16, II Satz 7.3]. 
Lemma 2.12 For every $n$ and every prime power $q$, the group $\operatorname{GL}(U), U=V(n, q)$, contains Singer groups. Moreover:

(a) Every two Singer groups are conjugate in $\operatorname{GL}(U)$.

(b) Let $S=\langle\sigma\rangle$ be a Singer group. Then, $N_{\mathrm{GL}(U)}(S)=S\langle\phi\rangle$, $\phi$ has order $n$ and can be chosen such that $\sigma^{\phi}=\sigma^{q}$.

(c) Let $A \leq \mathrm{GL}(U)$ be an irreducible abelian group. Then, $A$ is cyclic and $C_{\mathrm{GL}(U)}(A)$ is a Singer group.

Let $p$ be a prime and $n$ a positive integer. We call $0 \leq k, m<p^{n}-1$ equivalent and write $k \sim m$, iff there exist a nonnegative integer $s$, such that

$$
m \equiv p^{s} k \quad\left(\bmod p^{n}-1\right)
$$

Usually, one calls the equivalence classes cyclotomic cosets of $p$ modulo $n$. We call a cyclotomic coset long, if it has size $n$, and short otherwise.

Let $F=\mathbb{F}_{p^{n}}$, i.e., $Z=\left(F^{*}, \cdot\right)$ is a cyclic group of order $p^{n}-1$. For $1 \leq k<p^{n}-1$, define a representation $D^{k}: Z \rightarrow \operatorname{GL}_{\mathbb{F}_{p}}(F)$, by $u D^{k}(b)=b^{k} u, u \in F, b \in Z$. Clearly, the eigenvalues of $D^{k}(b)$ lie in $F$; i.e., the degree of the minimal polynomial of a $D^{k}(b)$ is a divisor $d$ of $n$. As a direct consequence of Lemma 2.12, one has:

Lemma 2.13 Let $\zeta$ be a generator of $Z$.

(a) The following statements are equivalent.

(1) $F$ as a $\mathbb{F}_{p} Z$-module for the representation $D^{k}$ splits into a direct sum of $n / d$ isomorphic, simple modules all of degree $d$.

(2) The minimal polynomial of $D^{k}(\zeta)$ has degree $d$.

(b) Every irreducible representation of $Z$ over $\mathbb{F}_{p}$ is isomorphic to a simple constituent of some representation $D^{k}, 0 \leq k<p^{n}-1$.

(c) The representation $D^{k}$ is irreducible, iff $k$ lies in a long coset modulo $p$, and $D^{k}$ is faithful, iff $\left(k, p^{n}-1\right)=1$.

(d) The irreducible constituents of $D^{k}$ and $D^{\ell}$ are isomorphic, iff $\ell \equiv p^{a} k\left(\bmod p^{n}-\right.$ 1) for some integer $a$, i.e., the irreducible $\mathbb{F}_{p} Z$-representations are in one-to-one correspondence with the cyclotomic cosets of $p$ modulo $n$.

For $0 \leq k<n$ and $a \in F$, define the $\mathbb{F}_{p}$-linear mapping $T_{k}(a) \in \operatorname{End}(F)$ by

$$
x T_{k}(a)=a x^{p^{k}} .
$$

It is well known that an $\mathbb{F}_{p}$-linear operator $\Phi$ on $F$ can be written uniquely in the form

$$
\Phi=T((a))=\sum_{i=0}^{n-1} T_{i}\left(a_{i}\right)
$$

with $a=\left(a_{0}, \ldots, a_{n-1}\right) \in F^{n}$. A Singer group $Z$ can be identified with $T_{0}\left(F^{*}\right)$. Also $T_{0}(\zeta)$ is a Singer cycle for $\zeta$ primitive in $F$. Set $\Phi=T_{1}(1)$. Then, $T_{0}(\zeta)^{\Phi}=$ $\Phi^{-1} T_{0}(\zeta) \Phi=T_{0}(\zeta)^{p}$ and $N_{\mathrm{GL}(F)}(Z)=Z\langle\Phi\rangle$. 
The space $E=\operatorname{End}_{\mathbb{F}_{p}}(F)$ is a $\mathbb{F}_{p} Z$-module for the representation $D^{(k, \ell)}: Z \rightarrow$ $\operatorname{GL}(E)$, where $\Phi D^{(k, \ell)}(u)=T_{0}\left(u^{k}\right)^{-1} \Phi T_{0}\left(u^{\ell}\right), \Phi \in E, u \in Z$, which is induced by $D^{k}$ and $D^{\ell}$. The next result is [10, Lemma 2.15]:

Lemma 2.14 We use the preceding notation and consider $E=\operatorname{End}_{\mathbb{F}_{p}}(F)$ as a $\mathbb{F}_{p} Z$ module for the representation $D^{(k, \ell)}$.

(a)

$$
E=T_{0}(F) \oplus T_{1}(F) \oplus \cdots \oplus T_{n-1}(F)
$$

is a decomposition into $\mathbb{F}_{p} Z$-modules.

(b) The $\mathbb{F}_{p} Z$-module $T_{i}(F)$ affords the representation $D^{\ell-p^{i} k}$, with the identification of $F$ and $T_{i}(F)$ via $a \mapsto T_{i}(a)$ and the action $T_{0}\left(u^{k}\right)^{-1} T_{i}(a) T_{0}\left(u^{\ell}\right)=$ $T_{i}\left(a D^{\ell-p^{i} k}(u)\right)=T_{i}\left(u^{\ell-p^{i} k} a\right)$ for $a \in F, u \in F^{*}$.

\subsection{On the $H^{1}$-cohomology of $\operatorname{Sp}(2 n, q)$}

For the investigation of Example 3.8, we need facts about the $H^{1}$-cohomology of $\operatorname{Sp}\left(2 n, 3^{a}\right)$. They follow from the more general main result of this section:

Theorem 2.15 Let $q$ be a prime power, $F=\mathbb{F}_{q}$ and $\tau \in \operatorname{Aut}(F)$. Let $G=\operatorname{Sp}(2 n, F)$, $n \geq 2$ be the symplectic group with the natural $F G$-module $V=V(2 n, q)$. Denote by $V^{\tau}$ the Frobenius twist of $V$ under $\tau$. Then, $\mathrm{H}^{1}\left(G, V^{\tau} \otimes V\right)=0$ for $n \geq 2$ except for $\tau=1$ and $(n, q)=(2,2),(2,3)$.

Remark 2.16 (a) Let $G$ be a finite group and $c: G \rightarrow V$ a 1-cocycle into the $F G$ module $V$. We write $c=c_{v}$, if $c$ is a 1-coboundary of the form $c(g)=v g-v$. Let $g, h \in G$ and $c(h)=0$. The following is obvious:

(1) $c(g) \in C_{V}(h)$ if $g$ and $h$ commute.

(2) Let $c=c_{v}$. Then $v \in C_{V}(h)$.

(b) Let $V$ be the standard $F G$-module for $G=\operatorname{Sp}(2 m, q), F=\mathbb{F}_{q}$. The following information can be found for instance in the proof of [21, Thm. 5.1]: The symmetric square $S^{2}(V)$ is simple, if $q$ is odd. If $q$ is even, then the socle $\operatorname{soc}\left(\left(S^{2}(V)\right) \simeq V^{\iota}\right.$ ( $\iota$ the Frobenius automorphism in $\left.\operatorname{Aut}\left(\mathbb{F}_{q}\right)\right)$ and $S^{2}(V) / \operatorname{soc}\left(\left(S^{2}(V)\right) \simeq \wedge^{2}(V)\right.$. Denote by $F$ the trivial $F \operatorname{Sp}(V)$-module. The module $\wedge^{2}(V)$ is the direct sum of $F$ and a simple module, if $(q, m)=1$. If $(q, m) \neq 1$, then $\wedge^{2}(V)$ has a socle series $0 \subset \operatorname{soc}^{1}\left(\wedge^{2}(V)\right) \subset \operatorname{soc}^{2}\left(\wedge^{2}(V)\right) \subset \wedge^{2}(V)$, such that $\operatorname{soc}^{1}\left(\wedge^{2}(V)\right) \simeq$ $\wedge^{2}(V) / \operatorname{soc}^{2}\left(\wedge^{2}(V)\right) \simeq F$, and $\operatorname{soc}^{2}\left(\wedge^{2}(V)\right) / \operatorname{soc}^{1}\left(\wedge^{2}(V)\right)$ is simple. Finally, $V \otimes V \simeq \wedge^{2}(V) \oplus S^{2}(V)$ if $q$ is odd. If $q$ is even, one has submodules $0 \subset A \subset$ $S \subset V \otimes V$ such that $A \simeq V \otimes V / S \simeq \wedge^{2}(V)$ and $V \otimes V / A \simeq S^{2}(V)$ and $S$ is dual to $S^{2}(V)$.

In the proof of the next lemma, we will denote by $V V$ either the symmetric square $S^{2}(V)$ of $V$ or $\wedge^{2}(V)$ the alternating square of $V$. Let $V=U \perp W$ be an orthogonal decomposition. Denote for $X, Y \in\{U, W\}$ by $X Y$ the submodule of $V V$, which is 
generated by the $x \cdot y$ (symmetric square) or $x \wedge y$ (alternating square), $x \in X, y \in Y$. For a non-degenerate subspace $X$, denote by $\operatorname{Sp}(X)$ the subgroup of $\operatorname{Sp}(V)$, which induces the symplectic group on $X$ and acts trivially on $X^{\perp}$. Note that $X X^{\perp} \simeq X \otimes X^{\perp}$ as a $F\left[\operatorname{Sp}(X) \times \operatorname{Sp}\left(X^{\perp}\right)\right]$-module.

Lemma 2.17 Let $q \leq 9$ be a prime power and $G=\operatorname{Sp}(2 n, q), V=V(2 n, q)$, $n \geq 2$. Then, $\mathrm{H}^{1}\left(G, S^{2}(V)\right)=\mathrm{H}^{1}\left(G, \wedge^{2}(V)\right)=0$ except for $\mathrm{H}^{1}\left(G, \wedge^{2}(V)\right)=\mathbb{F}_{q}$, $(n, q)=(2,2),(2,3)$.

Proof We have $\mathrm{H}^{1}(\mathrm{Sp}(V), V V) \simeq \operatorname{Ext}_{F \mathrm{Sp}(V)}(F, V V)$. For $n \leq 3$, the groups $\operatorname{Ext}_{F \operatorname{Sp}(V)}(F, V V)$ can be quickly computed with MAGMA [3], showing for $n \leq 3$ the assertion of the lemma. The remaining cases are verified by induction.

First, we assume $q=p=2$ or 3 . Let $\operatorname{dim} V \geq 8$ and $V=U \perp W, \operatorname{dim} U=2$. Set $K=O^{p}(\operatorname{Sp}(U))\left(\simeq \mathrm{C}_{3}\right.$ for $p=2$ and $\simeq \mathrm{Q}_{8}$ for $\left.p=3\right)$ and let $c: \operatorname{Sp}(V) \rightarrow V$ a 1-cocycle. We have $V V=U U \oplus U W \oplus W W$. Denote by $c_{X Y}$ the part of $c$, which lies in $X Y$.

We have $c_{W W}(K)=0$ as $W W$ is the trivial $K$-module and as $K$ has no subgroup of index $p$. As $K$ is a $p^{\prime}$-group, we can adjust $c$ by a coboundary $c_{w}, w \in U U \oplus U W$, such that $c(K)=0$. By induction, we can adjust $c$ a coboundary $c_{z}, z \in W W$, such that $c_{W W}(\operatorname{Sp}(W))=0$. Since $c_{z}(K)=0$, we still have $c(K)=0$. Then, $c_{U W}(\operatorname{Sp}(W))=0$ by (1), as $K$ has a trivial centralizer in $U W$ and $c_{U U}(\operatorname{Sp}(W))=0$ as $\operatorname{Sp}(W)$ has no subgroup of index $p$. Hence, $c(\operatorname{Sp}(W))=0$.

Let $W=W_{1} \perp W_{2}, \operatorname{dim} W_{1}=4$ and set $R=U \perp W_{1}$. We have $c_{R W_{2}}(\operatorname{Sp}(R))=$ 0 , as $C_{R W_{2}}\left(\operatorname{Sp}\left(W_{2}\right)\right)=0$ and $c\left(\operatorname{Sp}\left(W_{2}\right)\right)=0$ (note $W_{2} \subseteq W$ ). Also $c_{W_{2} W_{2}}(\operatorname{Sp}(R))=$ 0 , as $W_{2} W_{2}$ is a trivial $R$-module and $\operatorname{Sp}(R)$ has no subgroup of index $p$. As $K \times$ $\operatorname{Sp}\left(W_{1}\right) \leq \operatorname{Sp}(R) \cap(K \times \operatorname{Sp}(W))$, one has $c_{R R}\left(K \times \operatorname{Sp}\left(W_{1}\right)\right)=0$. We distinguish the cases (i) $p=3, V V=S^{2}(V)$, (ii) $p=2, V V=S^{2}(V)$, and (iii) $V V=\wedge^{2}(V)$.

By Remark 2.16(b): In case (i), the centralizer of $K \times \operatorname{Sp}\left(W_{1}\right)$ in $R R$ is trivial. In case (ii), the centralizer of $K \times \mathrm{Sp}\left(W_{1}\right)$ in $R R$ is $C_{U U}(K)$. In case (iii) the centralizer of $K \times \operatorname{Sp}\left(W_{1}\right)$ in $R R$ is $U U \oplus C_{W_{1} W_{1}}\left(\operatorname{Sp}\left(W_{1}\right)\right)$. In the latter case, we have $\operatorname{dim} U U=$ $\operatorname{dim} C_{W_{1} W_{1}}\left(\operatorname{Sp}\left(W_{1}\right)\right)=C_{R R}(\operatorname{Sp}(R))=1$ and $C_{R R}(\operatorname{Sp}(R))$ lies diagonally in $U U \oplus$ $C_{W_{1} W_{1}}\left(\operatorname{Sp}\left(W_{1}\right)\right)$.

We know that the restriction $\tilde{c}$ of $R$ to $c_{R R}$ is a 1-coboundary. By (2), we conclude in case (i) $\tilde{c}(R)=0$. Also (2) implies $\tilde{c}(z)=v z-v, z \in \mathrm{Sp}(R)$ for some $v \in U U$ in case (ii) and in case (iii) we have $\tilde{c}(z)=v z-v$ for some $v \in U U \oplus C_{W_{1} W_{1}}\left(\operatorname{Sp}\left(W_{1}\right)\right)$. Note that in case (iii) $v z-v=\left(v+v_{1}\right) z-\left(v+v_{1}\right)$ for $v_{1} \in C_{R R}(\operatorname{Sp}(R))$ and $z$ in $R$. Hence, we can choose $v \in U U$ in case (ii) as well as in case (iii). Set $c^{\prime}=c$ in case (i) and $c^{\prime}=$ $c-c_{v}$ in cases (ii) and (iii). By definition $c_{R R}^{\prime}(\operatorname{Sp}(R))=0$ and as $\operatorname{Sp}(W)$ centralizes $U U$, we have $c^{\prime}(\operatorname{Sp}(W))=c(\operatorname{Sp}(W))=0$. Again, $c_{W_{2} W_{2}}^{\prime}(\operatorname{Sp}(R))=0$, as $W_{2} W_{2}$ is a trivial $R$-module and $\operatorname{Sp}(R)$ has no subgroup of index $p$. Then, $c_{R W_{2}}^{\prime}(\operatorname{Sp}(R))=0$, since $c^{\prime}\left(\operatorname{Sp}\left(W_{2}\right)\right)=0$ and as $\operatorname{Sp}\left(W_{2}\right)$ has a trivial centralizer in $R W_{2}$. We conclude $c^{\prime}(R)=0$. Hence $c^{\prime}=0$, as $\operatorname{Sp}(W)$ and $\operatorname{Sp}(R)$ generate $\operatorname{Sp}(V)$.

For $4 \leq q \leq 9$, we can start our induction already with $\operatorname{dim} V \geq 6$ and $K=\operatorname{Sp}(U)$. All arguments of the previous section carry over.

I owe Sin [23] the proofs of the next two results. 
Lemma 2.18 Let $n>2, q=p^{t}>4$ a prime power, and $1 \neq \tau \in \operatorname{Aut}(F)$, where $F=\mathbb{F}_{q}$. Let $G=\operatorname{Sp}(2 n, q), n>1$ and $V$ be the standard $F G$-module. Then, $\mathrm{H}^{1}\left(G, V^{\tau} \otimes V\right)=0$.

Proof (P. Sin) Let $e_{1}, \ldots, e_{n}, f_{1}, \ldots, f_{n}$ be a symplectic basis with $\left(e_{i}, f_{j}\right)=\delta_{i, j}$, i.e., the associated Gram matrix has the shape $\left(\begin{array}{cc}0 & 1_{n \times n} \\ -1_{n \times n} & 0\end{array}\right)$. Let $P=G_{W}$ be the stabilizer of the isotropic space spanned by the $e_{i}$ 's. Then, $P=A L$ is a semidirect product, with

$$
L=\left\{\left(\begin{array}{cc}
g & 0 \\
0 & g^{-t}
\end{array}\right) \mid g \in \mathrm{GL}(W)\right\} \quad \text { and } \quad A=\left\{\left(\begin{array}{cc}
1_{n \times n} & 0 \\
S & 1_{n \times n}
\end{array}\right) \mid S=S^{t}\right\}
$$

is elementary abelian and in fact is an $F$-vector space with scalar multiplication induced by $Z(L) \simeq F^{*}$. The action of $L$ on $A$ by conjugation corresponds to the action of $\operatorname{GL}(n, F)$ on the space $\operatorname{Sym}(n, n, F)$ of symmetric $m \times m$-matrices by the representation $G \times \operatorname{Sym}(n, n, F) \ni(g, S) \mapsto g^{t} \operatorname{Sg} \in \operatorname{Sym}(n, n, F)$.

As a $F P$-module $V$ is the nonsplit extension of $W$ with $W^{*}$ ( $F P$ and $F L$ have the same simple modules as $O_{p}(P)=A$ ). Thus, the $F P$-module $V^{\tau} \otimes V$ has four composition factors:

$$
W^{\tau} \otimes W, \quad W^{\tau} \otimes W^{*}, \quad\left(W^{*}\right)^{\tau} \otimes W, \quad\left(W^{*}\right)^{\tau} \otimes W^{*},
$$

which are simple by the Steinberg tensor product theorem $\left(W^{*}\right.$ is the dual of $W$ ). The restriction map $\mathrm{H}^{1}\left(G, V^{\tau} \otimes V\right) \rightarrow \mathrm{H}^{1}\left(P, V^{\tau} \otimes V\right)$ is injective as $|G: P|$ is coprime to $p$, and we shall prove that its codomain is zero. From the long exact sequence of cohomology, it suffices to show that $\mathrm{H}^{1}(P, X)=0$ for each composition factor $X$ in (2). We apply the inflation restriction sequence

$$
0 \rightarrow \mathrm{H}^{1}\left(L, X^{A}\right) \rightarrow \mathrm{H}^{1}(P, X) \rightarrow \mathrm{H}^{1}(A, X)^{L} .
$$

In the first term of (3), we have $X=X^{A}$. For each $X$ in (2), we follow easily from the fact that $Z(L)$ acts non-trivially on $X$ (this argument fails for $q=4$ ). For the third term in (3), since $A$ is elementary abelian and acts trivially on $X$, we have

$$
\mathrm{H}^{1}(A, X)^{L} \simeq \operatorname{Hom}_{\mathbb{F}_{p} L}(A, X)
$$

As an $\mathbb{F}_{p} L$-module, $A$ is simple and the modules $X$ are also simple $\mathbb{F}_{p} L$-modules except when $\tau$ is an involution and $W^{\tau} \otimes W$ and $\left(W^{*}\right)^{\tau} \otimes W^{*}$ split into two simple $\mathbb{F}_{p} L$-modules of the same dimension. Comparing dimensions, we see that the third term of (3) is zero for all $X$ in (2).

P. Sin argues similarly in the case $q=4$ and, however, replaces the parabolic subgroup $P$ by a stabilizer of a 1 -space, and he obtains:

Lemma 2.19 Let $G=\operatorname{Sp}(2 n, 4), n \geq 1, \tau$ the involution in $\operatorname{Aut}\left(\mathbb{F}_{4}\right)$ and $V$ be the standard FG-module. Then, $\mathrm{H}^{1}\left(G, V^{\tau} \otimes V\right)=0$. 
Alternatively, one can follow the pattern of the proof of Lemma 2.17 to obtain a verification of Lemma 2.19.

Proof (Of Theorem 2.15) For $\tau \neq 1$, the assertion of the theorem follows from Lemmas 2.18 and 2.19. So assume $\tau=1$. We have $\mathrm{H}^{1}\left(G, S^{2}(V)\right)=0$ for $q \leq 9$ by Lemma 2.17 and for $q>9$ by [26]. Also $\mathrm{H}^{1}\left(G, \wedge^{2}(V)\right)=0$ for $q \leq 9$ (except $(n, q)=(2,2),(2,3))$ by Lemma 2.17 and for $q>9$ we have by [17] $\mathrm{H}^{1}(G, L)=0$, where $L$ is the non-trivial simple composition factor of $\wedge^{2}(V)$. Together with Remark 2.16(b) and the long exact sequence of cohomology, we have again $\mathrm{H}^{1}\left(G, \wedge^{2}(V)\right)=0$. If $q$ is odd, we are done by Remark 2.16(b) and for $q$ even we use again the long exact sequence of cohomology in combination with Remark 2.16(b).

\section{Some examples}

In this section, we introduce classes of $p$-ary bent functions closely related to quadratic forms. In the subsequent sections, we shall consider equivalence of these bent functions and deal with their automorphism groups. For $p=2$, these examples were already investigated in [9] and their affine equivalence and affine automorphisms were determined.

\subsection{Perturbations of quadratic forms}

A construction of bent functions. We recall two constructions of bent functions. For $i=1,2$, let $V_{i}$ be finite-dimensional $\mathbb{F}_{p}$-spaces and $g_{i}: V_{i} \rightarrow \mathbb{F}_{p}$ bent functions. We denote by $g_{1} \oplus g_{2}: V_{1} \oplus V_{2} \rightarrow \mathbb{F}_{p}$, the direct sum of $g_{1}$ and $g_{2}$, the bent function defined by $\left(g_{1} \oplus g_{2}\right)\left(x_{1}+x_{2}\right)=g_{1}\left(x_{1}\right)+g_{2}\left(x_{2}\right), x_{i} \in V_{1}$ (cf. [19, Prop. 3]).

Let $W$ be a finite-dimensional $\mathbb{F}_{p}$-space, $Q$ a non-degenerate quadratic form on $W$, let $(\cdot, \cdot)$ be the polarization of $Q$ and let $W=W_{1} \oplus W_{2}$ be a decomposition into two maximal totally singular subspaces. For a permutation $\rho$ of $W_{2}$ and a function $h: W_{2} \rightarrow \mathbb{F}_{p}$, define the Maiorana-McFarland bent function or Maiorana function $\mu=\mu_{\rho, h}: W \rightarrow \mathbb{F}_{p}$ by $\mu\left(x_{1}+x_{2}\right)=\left(x_{1}, x_{2}^{\rho}\right)+h\left(x_{2}\right)$ (cf. [7]).

Let $V$ be a finite-dimensional $\mathbb{F}_{p}$-space, $Q$ a non-degenerate quadratic form on $V$ and $W_{0}$ be a non-degenerate subspace of $V$. Let $W_{0}^{\perp}=W_{1} \oplus W_{2}$ be a decomposition into two maximal totally singular subspaces. Let $g, k: W_{0} \rightarrow \mathbb{F}_{p}$ be arbitrary bent functions and $\mu=\mu_{\rho, h}: W_{0}^{\perp}=W_{1} \oplus W_{2} \rightarrow \mathbb{F}_{p}$ a Maiorana function. Assume that $\rho$ fixes 0 . Define $f=f(g, k, \mu): V \rightarrow \mathbb{F}_{p}$ by

$$
f(x)= \begin{cases}g\left(x_{0}\right), & x \in W_{0} \oplus W_{1}, \\ (k \oplus \mu)(x), & x \notin W_{0} \oplus W_{1},\end{cases}
$$

where $x_{0}$ is the projection of $x \in W_{0} \oplus W_{1}$ into $W_{0}$. We have:

Proposition $3.1 f(g, k, \mu)$ is a bent function. 
Proof Let $0 \neq b \in V$. We have to show $S(f, b)=\sum_{x \in V} \zeta_{p}^{f(x)-f(x+b)}=0$. We write elements $x, b$ in $V$ as $x=x_{0}+x_{1}+x_{2}, b=b_{0}+b_{1}+b_{2}$ with $x_{i}, b_{i} \in W_{i}$, $i=0,1,2$. Set $f^{\prime}=k \oplus \mu$.

CASE $b_{2}=0$. We have for $x_{2}=0$

$$
\begin{aligned}
\sum_{x_{0}, x_{1}} \zeta_{p}^{f(x)-f(x+b)} & =\left|W_{1}\right| \sum_{x_{0}} \zeta_{p}^{g\left(x_{0}\right)-g\left(x_{0}+b_{0}\right)} \\
& =\left|W_{1}\right| \sum_{x_{0}} \zeta_{p}^{k\left(x_{0}\right)-k\left(x_{0}+b_{0}\right)}=\sum_{x_{0}, x_{1}} \zeta_{p}^{f^{\prime}(x)-f^{\prime}(x+b)}
\end{aligned}
$$

since $0^{\rho}=0$. As $f$ and $f^{\prime}$ coincide on $V-\left(W_{0} \oplus W_{1}\right)$, we get $S(f, b)=S\left(f^{\prime}, b\right)=0$.

CASE $b_{2} \neq 0$. Then, $S(f, b)=A+B+C$ with

$$
\begin{aligned}
A & =\sum_{x_{0}, x_{1}, x_{2} \neq 0,-b_{2}} \zeta_{p}^{k\left(x_{0}\right)-k\left(x_{0}+b_{0}\right)+\mu\left(x_{1}+x_{2}\right)-\mu\left(x_{1}+b_{1}+x_{2}+b_{2}\right)}, \\
B & =\sum_{x_{0}, x_{1}} \zeta_{p}^{g\left(x_{0}\right)-k\left(x_{0}+b_{0}\right)-\mu\left(x_{1}+b_{1}+b_{2}\right)}, \quad\left(x_{2}=0\right),
\end{aligned}
$$

and

$$
C=\sum_{x_{0}, x_{1}} \zeta_{p}^{k\left(x_{0}\right)+\mu\left(x_{1}-b_{2}\right)-g\left(x_{0}+b_{0}\right)}, \quad\left(x_{2}=-b_{2}\right)
$$

Now $\mu\left(x_{1}+x_{2}\right)-\mu\left(x_{1}+b_{1}+x_{2}+b_{2}\right)=\left(x_{1}, x_{2}^{\rho}-\left(x_{2}+b_{2}\right)^{\rho}\right)+c$ with a function $c=c\left(x_{2}\right)$ in $x_{2}$. As a function of $x_{1}$, this expression has the form $x_{1} \lambda+c, \lambda$ a nontrivial functional on $W_{1}$, since $\rho$ is a permutation. So for a fixed $x_{2} \neq 0,-b_{2}$, we get

$$
\begin{aligned}
& \sum_{x_{0}, x_{1}} \zeta_{p}^{k\left(x_{0}\right)-k\left(x_{0}+b_{0}\right)+\mu\left(x_{1}+x_{2}\right)-\mu\left(x_{1}+b_{1}+x_{2}+b_{2}\right)}=\left(\zeta_{p}^{c} \sum_{x_{1}} \zeta_{p}^{x_{1} \lambda}\right) \\
& \cdot\left(\sum_{x_{0}} \zeta_{p}^{k\left(x_{0}\right)-k\left(x_{0}+b_{0}\right)}\right)=0,
\end{aligned}
$$

i.e., $A=0$. We have $W_{1}=W^{\prime} \oplus \mathbb{F}_{p} c_{1}$ with $W^{\prime}=W_{1} \cap\left(\mathbb{F}_{p} b_{2}^{\rho}\right)^{\perp},\left(c_{1}, b_{2}^{\rho}\right)=1$. Write $x_{1}=y+a c_{1}, y \in W^{\prime}, a \in \mathbb{F}_{p}$. Then, $\mu\left(x_{1}+b_{1}+b_{2}\right)=a+\left(b_{1}, b_{2}^{\rho}\right)+h\left(b_{2}\right)$. So

$$
B=\left(\left|W^{\prime}\right| \zeta_{p}^{\left(b_{1}, b_{2}^{\rho}\right)+h\left(b_{2}\right)} \sum_{a \in \mathbb{F}_{p}} \zeta_{p}^{a}\right)\left(\sum_{x_{0} \in W_{0}} \zeta_{p}^{g\left(x_{0}\right)-k\left(x_{0}+b_{0}\right)}\right)=0
$$

Similarly, one shows $C=0$.

Remark 3.2 (a) One referee of this paper points out a connection between the functions $f(g, k, \mu)$ and a criterion for bentness: $f: \mathbb{F}_{p}^{n} \times \mathbb{F}_{p}^{s} \rightarrow \mathbb{F}_{p}$ is bent, if for all $y \in \mathbb{F}_{p}^{s}$ 
the function $f_{y}: \mathbb{F}_{p}^{n} \rightarrow \mathbb{F}_{p}$ defined by $f_{y}(x)=f(x, y)$ is $s$-plateaued and if the supports of the functions $f_{y}$ are pairwise disjoint (see, for instance, for [7] for the definition of "s-plateaued" and the criterion). For $f=f(g, k, \mu)$ and $x_{2} \in W_{2}$, clearly $f_{x_{2}}\left(x_{0}, x_{1}\right)=f\left(x_{0}, x_{1}, x_{2}\right)$ defines a dim $W_{2}$-plateaued function. So for many choices of $g, k$ and $\mu$, the criterion is applicable.

(b) Proposition 3.1 allows the construction of many bent functions. We consider the case, where $g, k$ and $\mu$ are quadratic forms closely related to $Q$. In such cases, large parts of the automorphism group of $Q$ are also automorphisms of $f$ and one can hope in turn to compute the full automorphism group of $f$.

We will pick out some special examples, which we call of parabolic type in order to be consistent with the denomination of analogical examples in [9]. There is no special reason for this choice of examples other than to exhibit some group-theoretic machinery, which sometimes can solve equivalence and automorphism problems.

Assume the notation of Proposition 3.1 and set $W=W_{0} \oplus W_{1}$. Let $\Pi \in \mathrm{GL}\left(W_{0}\right)$ and $a, b \in \mathbb{F}_{p}, b \neq 0$. Define $Q_{W, \Pi, a}^{b}: V \rightarrow \mathbb{F}_{p}$ by

$$
Q_{W, \Pi, a}^{b}\left(x_{0}+x_{1}+x_{2}\right)= \begin{cases}Q\left(x_{0} \Pi\right), & x_{2}=0 \\ b\left(Q\left(x_{0}\right)+\left(x_{1}, x_{2}\right)\right)+a, & x_{2} \neq 0\end{cases}
$$

Note that $b\left(Q\left(x_{0}\right)+\left(x_{1}, x_{2}\right)\right)=b Q\left(x_{0}+x_{1}+x_{2}\right)$. By Proposition 3.1, $Q_{W, \Pi, a}^{b}$ is bent. It has by construction a "large" group of automorphisms:

Corollary 3.3 Let $f=Q_{W, \Pi, a}^{b}$ be defined as above.

(a) $\mathbf{A}(f)$ contains a group $\mathcal{Q} \simeq O_{p}\left(\mathrm{O}(V, Q)_{W}\right)$ (the p-radical of the stabilizer of $W$ in $\mathrm{O}(V, Q)$ ).

(b) $\mathbf{A}(f)$ contains a group $H \simeq \mathrm{GL}\left(W_{1}\right)$. Moreover, $H$ leaves the decomposition $V=W_{0} \oplus W_{1} \oplus W_{2}$ invariant, $C_{V}(H)=W_{0}$, H induces the natural representation of $\mathrm{GL}\left(W_{1}\right)$ on $W_{1}$ and the dual representation on $W_{2}$.

(c) $\beta(\operatorname{rad}(W)) \leq \mathbf{E}(f)$.

Proof (a) Let $\Phi \in O_{p}\left(\mathrm{O}(V, Q)_{W}\right)$. If $x \notin W$, then $x \Phi=x+w, w \in W$ and $Q(x)=Q(x \Phi)$, showing $f(x)=b Q(x)+a=b Q(x \Phi)+a=f(x \Phi)$. If $x \in W$, then $x=x_{0}+x_{1}, x_{i} \in W_{i}$, and $x \Phi=x_{0}+x_{1}^{\prime}$ for some $x^{\prime} \in W_{1}$. Again $f(x)=f\left(x_{0}+x_{1}\right)=f\left(x_{0}+x_{1}^{\prime}\right)=f(x \Phi)$ and $\operatorname{diag}(\Phi, 1) \in \mathbf{A}(f)$ follows.

(b) Let $W=W_{0} \oplus W_{1}$ and $\Pi \in \mathrm{GL}\left(W_{0}\right)$ as in the definition of $f=Q_{W, \Pi, a}^{b}$. One observes that $\Phi: \operatorname{GL}\left(W_{1}\right) \ni \sigma \mapsto \Phi(\sigma)=\operatorname{diag}\left(1_{W_{0}}, \sigma,\left(\sigma^{-1}\right)^{*}\right) \in \mathrm{O}(V, Q)$ is an injection. A routine calculation shows $f(x \Phi(\sigma))=f(x)$ for $x \in V$. So $H=\left\{\operatorname{diag}(\Phi(\sigma), 1) \mid \sigma \in \mathrm{GL}\left(W_{1}\right)\right\} \leq \mathbf{A}(f)$.

(c) Let $u$ be in $\operatorname{rad}(W)$. We have $f(u)=0$. If we set $x \lambda_{u}=f(x+u)-f(x)-$ $f(u)=f(x+u)-f(u)$, we get $x \lambda_{u}=0$ if $x \in W$ and $x \lambda_{u}=b(u, x)$ for $x \notin W$. So $x \lambda_{u}=b(u, x)$ holds globally. Equation (1) of Remark 2.5 becomes $\left(x_{0}+x_{1}+x_{2}, z\right) \beta(u)=\left(x_{0}+\left(x_{1}+u\right)+x_{2}, z+x \lambda_{u}\right)$. We compute $(x, f(x)) \beta(u)=$ $(x+u, f(x+u)$ and obtain $\beta(u) \in \mathbf{E}(f)$.

Some special cases of Construction (4) will be studied in the sequel. 
Example 3.4 Let $Q$ be a non-degenerate quadratic form on $V=V(n, p)$. Let $W$ be a subspace such that $\operatorname{rad}(W)=W^{\perp}$ is a non-trivial totally singular space. For $a, b \in \mathbb{F}_{p}, b \neq 0$, define $Q_{W, a}^{b}: V \rightarrow \mathbb{F}_{p}$ as $Q_{W, 1_{W_{0}}, a}^{b}$, i.e.,

$$
Q_{W, a}^{b}(x)= \begin{cases}Q(x), & x \in W, \\ b Q(x)+a, & x \notin W .\end{cases}
$$

We call $Q_{W, a}^{b}$ a

\section{standard parabolic bent function.}

Clearly, for $a=0$ and $b=1$, we have $Q=Q_{W, a}^{b}$, while for $W=W^{\perp}$ of dimension $n / 2$ ( $n$ has to be even) $Q_{W, 0}^{b}=b Q$ is again a quadratic form. Set $f=Q_{W, a}^{b}$. Let $p>2$ and $K=\mathrm{O}(V, Q)_{W}$ be the stabilizer of $W$ in the orthogonal group. Clearly, any transformation $\Phi \in K$ fixes $\operatorname{rad}(W)$ too. We consider $K$ as a subgroup of GL(U), $\mathcal{U}=V \times \mathbb{F}_{p}$ by extending $\Phi$ to $\operatorname{diag}(\Phi, 1)$. It is easy to see that $K \leq \mathbf{A}(f)$. We have already seen in Corollary 3.3, that $\mathbf{E}(f)$ contains a $\operatorname{group} \beta(\operatorname{rad}(W)) \simeq(\operatorname{rad}(W),+)$.

Assume next $b=1$. Let $w \in W$. We observe $f(x+w)-f(x)-f(w)=(x, w)$ for all $x \in V$. Define $\beta(w)$ as in Eq. (1) of Remark 2.5, and we have $\beta(w) \in \mathbf{E}(f)$, i.e., $\beta(W) \leq \mathbf{E}(f)$.

Remark 3.5 Clearly, we have $Q_{W, a}^{b}=b Q+(1-b) Q_{W}+a\left(1-\chi_{W}\right)$, where $\chi_{W}$ is the characteristic function of $W$. Choosing $Q$ as in Remark 2.7(1)-(3) and $W=\left\langle e_{1}, \ldots, e_{k}, \ldots, e_{n-k}\right\rangle, k \leq\left\lfloor\frac{n}{2}\right\rfloor$, this allows us to express $Q_{W, a}^{b}$ as a polynomial. If $W=W^{\perp}$, then $Q \chi_{W^{\perp}}=0$, and if $W$ is a hyperplane, then $\chi_{W}=$ const $\cdot \prod_{0 \neq e \in \mathbb{F}_{p}}\left(X_{n}-e\right)$. So if $p>2$, then in the following cases $Q_{W, a}^{b}$ is a quadratic form:

(1) $W=W^{\perp}\left(n\right.$ is even) and $a=0$ (i.e., $Q_{W, 0}^{b}=b Q$ ).

(2) $W$ is a hyperplane, $b=1$ and $p=3$.

(3) $(a, b)=(0,1)$ (i.e., $\left.Q_{W, a}^{b}=Q\right)$.

We call $Q_{W, a}^{b}$ a proper standard parabolic bent function, if it is not a quadratic form. Finally, if $W=W^{\perp}$, then $Q_{W, a}^{b}$ is equivalent to $Q_{W, b^{-1} a}^{1}$. So we will make the convention $b=1$, if $W=W^{\perp}$.

Example 3.6 Let $Q$ be a non-degenerate quadratic form on $V=V(n, p)$. Let $W$ be a subspace such that $W_{1}=\operatorname{rad}(W)=W^{\perp}$ is a non-trivial totally singular space and $V=W_{0} \oplus W_{1} \oplus W_{2}$ with $W=W_{0} \oplus W_{1}$ and $W_{2} \subseteq W_{0}^{\perp}$ totally singular. For $\Pi \in \mathrm{GL}\left(W_{0}\right)$, define $Q_{W, \Pi}: V \rightarrow \mathbb{F}_{p}$ as $Q_{W, \Pi, 0}^{1}$, i.e.,

$$
Q_{W, \Pi}\left(x_{0}+x_{1}+x_{2}\right)= \begin{cases}Q\left(x_{0} \Pi\right), & x_{2}=0 \\ Q\left(x_{0}\right)+\left(x_{1}, x_{2}\right), & x_{2} \neq 0\end{cases}
$$

We call a bent function $Q_{W, \Pi \text { a }}$

twisted parabolic bent function. 
By Corollary 3.3, we already know

$$
\mathcal{Q}=O_{p}\left(\mathrm{GO}(V, Q)_{W}\right) \leq \mathbf{A}\left(Q_{W, \Pi}\right), \quad \beta\left(W_{1}\right) \leq \mathbf{E}\left(Q_{W, \Pi}\right)
$$

and

$$
H \simeq \mathrm{GL}\left(W_{1}\right) \leq \mathrm{GO}(V, Q)_{W_{0}, W_{1}, W_{2}} \cap \mathbf{A}\left(Q_{W, \Pi}\right)
$$

Remark 3.7 If $\Pi \in \mathrm{O}\left(W_{0}, Q_{W_{0}}\right)$, then $Q=Q_{W, \Pi}$ is a quadratic form. Conversely, if $Q_{W, \Pi}$ is a quadratic form, then $\Pi \in \mathrm{O}\left(W_{0}, Q_{W_{0}}\right)$ : Let $[\cdot, \cdot]$ be the bilinear form associated with this quadratic form. Then, by definition

$$
\left[w, w^{\prime}\right]=\left(w \Pi, w^{\prime} \Pi\right) \quad w, w^{\prime} \in W_{0} .
$$

Let $0 \neq v \in W_{2}$. Then $v, v+w^{\prime}, v+w+w^{\prime} \notin W$, so that $[w, v]=Q(v+w)-$ $Q(w \Pi)-Q(v)$ and $\left[w, v+w^{\prime}\right]=Q\left(v+w+w^{\prime}\right)-Q(w \Pi)-Q\left(v+w^{\prime}\right)$ holds. Expanding the second equation and using the bilinearity of $[\cdot, \cdot]$, one obtains

$$
\left(w, w^{\prime}\right)=\left[w, w^{\prime}\right]=\left(w \Pi, w^{\prime} \Pi\right)
$$

and $\Pi \in \mathrm{O}\left(W_{0}, Q_{W_{0}}\right)$ follows. We call $Q_{W, \Pi}$ proper, if this function is not a quadratic form.

We give a variation of these constructions, which rests on fact that a certain cohomology group does not vanish.

\section{Some indecomposable $\operatorname{SL}\left(2,3^{n}\right)$-modules}

Let $F=\mathbb{F}_{q}, q=3^{n}, n \geq 2$, let $\sigma$ be the inverse of the Frobenius automorphism of $F$ and set $V=F^{4}$. We define in $\operatorname{GL}(V)$ the elements $h(v)=\operatorname{diag}\left(v^{\sigma}, v^{-\sigma}, v, v^{-1}\right) \in$ $\mathrm{GL}(V), v \in F^{*}, w_{1}=\operatorname{diag}(J, J), J=\left(\begin{array}{cc}0 & -1 \\ 1 & 0\end{array}\right)$, and

$$
x_{+}(u)=\left(\begin{array}{cccc}
1 & 0 & 0 & 0 \\
u^{\sigma} & 1 & 0 & 0 \\
0 & 0 & 1 & 0 \\
u^{2 \sigma} & -u^{\sigma} & u & 1
\end{array}\right)
$$

$u \in F$ and set $x_{-}(-u)=x_{+}(u)^{w_{1}}$. Define $H=\left\langle x_{+}(u), x_{-}(u) \mid u \in F\right\rangle$. A routine computation shows that the generators of $H$ satisfy the Steinberg relations for SL $(2, q)$, i.e., $H \simeq \operatorname{SL}(2, q)$ by $\left[24\right.$, Thm. 8]. Let $M=V(2, q)$ be the natural $\mathbb{F}_{q} \operatorname{SL}(2, q)$ module. Then, $M^{\sigma} \simeq W, W=F \times F \times 0 \times 0$ and $V / W \simeq M$. So $V$ is the extension of the module $M^{\sigma}$ by $M$. The explicit description of the non-vanishing elements in $\operatorname{Ext}_{F \mathrm{SL}(2, q)}\left(M^{\sigma}, M\right) \simeq \mathrm{H}^{1}\left(\mathrm{SL}(2, q), M^{\sigma} \otimes M^{*}\right) \simeq \mathrm{H}^{1}\left(\mathrm{SL}(2, q), M^{\sigma} \otimes M\right)$ (here $M^{*} \simeq M$, where $M^{*}$ is the dual module for $M$ ) in [2, Lemma 3.3] shows that $V$ is an indecomposable $\operatorname{FSL}(2, q)$-module. 
Example 3.8 Set as above $F=\mathbb{F}_{q}, q=3^{n}, n \geq 2, V=F^{4}$ and consider this indecomposable $F H$-module, $H=\operatorname{SL}(2, q)$ as an $\mathbb{F}_{3} H$-module. We do this by replacing each matrix entry $x$ of $F$ by $T_{0}(x)$, for instance $h(v)=$ $\operatorname{diag}\left(T_{0}\left(v^{\sigma}\right), T_{0}\left(v^{-\sigma}\right), T_{0}(v), T_{0}\left(v^{-1}\right)\right)$. Conjugating by $\operatorname{diag}\left(T_{1}(1), T_{1}(1), 1,1\right)$, the generators get the shape:

$$
\begin{aligned}
h(v) & =\operatorname{diag}\left(T_{0}(v), T_{0}\left(v^{-1}\right), T_{0}(v), T_{0}\left(v^{-1}\right)\right), \quad w(1)=\operatorname{diag}(J, J), \\
J & =\left(\begin{array}{cc}
0 & T_{0}(-1) \\
T_{0}(1) & 0
\end{array}\right),
\end{aligned}
$$

and

$$
x_{+}(u)=\left(\begin{array}{cccc}
1 & 0 & 0 & 0 \\
T_{0}(u) & 1 & 0 & 0 \\
0 & 0 & 1 & 0 \\
T_{1}\left(u^{2}\right) & T_{1}(-u) & T_{0}(u) & 1
\end{array}\right) .
$$

We define on the $\mathbb{F}_{3}$-space $V$ a quadratic form by $Q\left(x_{1}, x_{2}, x_{3}, x_{4}\right)=\operatorname{Tr}\left(x_{1} x_{4}-x_{2} x_{3}\right)$, where $\operatorname{Tr}: F \rightarrow \mathbb{F}_{3}$ is the trace map. Then, $W=F \times F \times 0 \times 0$ and $\widehat{W}=0 \times 0 \times F \times F$ are totally singular spaces. The element

$$
T=\left(\begin{array}{cccc}
1 & 0 & 0 & 0 \\
0 & 1 & 0 & 0 \\
T((a)) & T((b)) & 1 & 0 \\
T(((c)) & T((d)) & 0 & 1
\end{array}\right)
$$

lies in $\mathrm{O}(V, Q)$ and hence in the 3-radical $\mathcal{Q}=O_{3}\left(\mathrm{O}(V, Q)_{W}\right)$ of the parabolic subgroup $\mathrm{O}(V, Q)_{W}$, iff $T((d))=T((a))^{*}, T((b))^{*}=-T((b))$, and $T((c))^{*}=$ $-T((c))$. Here, $T((x))^{*}=\sum_{i=0}^{n-1} T_{i}\left(x_{n-i}^{3^{n-i}}\right)$ if $x=\left(x_{0}, \ldots, x_{n-1}\right)$. Note that $T((x))^{*}$ is the adjoint operator to $T((x))$ with respect to the bilinear form $F^{2} \ni(x, y) \mapsto$ $\operatorname{Tr}(x y)$ on $F$. A computation shows that the $h(v)$ 's and $w(1)$ lie in $\mathrm{O}(V, Q)$ but not $x_{+}(u), u \neq 0$, since $Q$ takes nonzero values on the orbit of $v_{4}=(0,0,0,1)$ under $X_{+}=\left\langle x_{+}(u) \mid u \in F\right\rangle$. Nevertheless, $X_{+}$normalizes the 3-radical $\mathcal{Q}$, i.e., $\mathcal{Q} H$ is a group, where $H=\left\langle X_{+}, w_{1}, h\left(F^{*}\right)\right\rangle$.

Any element $v \in V-W$ is mapped under $\mathcal{Q}$ to every element $v+w \in v+W$ with $Q(v)=Q(v+w)$. If $v_{3}=(0,0,1,0)$, then $C_{H}\left(v_{3}\right)=C_{H}\left(v_{3}+W\right)=X_{+}$, so that $V-W$ splits under $\mathcal{Q} H$ into three orbits $B_{0}, B_{1}, B_{2}$ of the same length. We choose the notation, such that $v_{4}=(0,0,0,1) \in B_{0}$.

Let $\sigma$ be an element in $\operatorname{Sym}(3)$. Define a function $f=Q^{\sigma}: V \rightarrow \mathbb{F}_{3}$ by $f(v)=0$ for $v \in W$ and $f(v)=i^{\sigma}$ if $v \in B_{i} \cap(V-W)$. Then, $f$ is a 3-ary bent function of Maiorana type: Let $0 \neq u \in \widehat{W}$. Then, $f$ is constant on $[u, \mathcal{Q}]+u=\left(W \cap\langle u\rangle^{\perp}\right)+u$. But $W \cap\langle u\rangle^{\perp}$ is a hyperplane of $W$. So $W \ni x \mapsto f(x, u)-f(0, u) \in \mathbb{F}_{3}$ is a linear functional (we identify $V$ with $W \times \widehat{W}$ and $W$ and $\widehat{W}$ with $F^{2}$ ). Hence, there exists a unique $0 \neq u^{\pi} \in \widehat{W}$, such that for all $w \in W$ the equation $(*) f(w, u)=\left(w, u^{\pi}\right)$ holds $((\cdot, \cdot)$ is the bilinear form induced by $Q)$. Setting $0^{\pi}=0$, then $\pi$ is a permutation on $\widehat{W}$ and equation (*) holds for all $(w, u) \in V$. 
We call $f=Q^{\sigma}$ of special parabolic type.

Set $\mathcal{U}_{0}=0 \times 0 \times 0 \times 0 \times \mathbb{F}_{p}$. By construction $H \mathcal{Q} \leq \mathbf{A}(f)$, where we extend the action of $H \mathcal{Q}$ on $V$ to $\mathcal{U}$ by setting $(H \mathcal{Q})_{\mathcal{U}_{0}}=1$. We observe that $\Theta$ with $\Theta_{V}=\operatorname{diag}\left(T_{1}(1), T_{1}(1), T_{1}(1), T_{1}(1)\right), \Theta_{\mathcal{U}_{0}}=1$ fixes $v_{3}$ and normalizes $H \mathcal{Q}$. Hence, $\Theta \in \mathbf{A}(f)$. Define $z(-1) \in \operatorname{GL}(\mathcal{U})$ by $z(-1)_{V}=\operatorname{diag}(1,-1,-1,1)$ and $z(-1) \mathcal{U}_{0}=$ 1. Then, $z(-1)$ fixes $v_{4}$ and normalizes $H \mathcal{Q}$. Hence, $\Theta, z(-1) \in \mathbf{A}(f)$, as $v_{3}$ and $v_{4}$ lie in the same $\mathcal{Q} H$-orbit. If $n$ is even, we pick $v \in F^{*}$ of order 4 and define the element $z(v) \in \operatorname{GL}(\mathcal{U})$ by $z(v)_{V}=\operatorname{diag}\left(T_{0}(-1), T_{0}(v), T_{0}(v), T_{0}(1)\right)$, and $z(v)_{\mathcal{U}_{0}}=$ -1 , i.e., $|z(v)|=4$. We see that $z(v)$ normalizes $H \mathcal{Q}$, and we recall $v_{4} \in B_{0}$. As $( \pm k, 0,0,0)+v_{4}$ (where $\left.\operatorname{Tr}(k)=1\right)$ represent the orbits $B_{1}$ and $B_{2}$ and as $z(v)$ interchanges these elements, we get $z(v) \in \mathbf{A}(f)$, iff $\sigma$ fixes 0 . So $N_{\mathbf{A}(f)}(H \mathcal{Q}) / H \mathcal{Q}$ contains a subgroup isomorphic to $\operatorname{Aut}(F) \mathrm{C}_{4}$ if $n$ is even and $\sigma$ fixes 0 . Otherwise, $N_{\mathbf{A}(f)}(H \mathcal{Q}) / H \mathcal{Q}$ contains a subgroup isomorphic to $\operatorname{Aut}(F) \mathrm{C}_{2}$.

We claim that $\beta(W) \leq \mathbf{E}(f)$ : As $\mathcal{Q} \leq \mathbf{A}(f)$, we see that for $(x, y, 0,1) \in W+v_{4}$ the function $f$ takes the value $f(x, y, 0,1)=e \operatorname{Tr}(x)+b$ for some $b, e \in \mathbb{F}_{3}, e \neq 0$. The scalars $b, e$ depend only on the choice of $\sigma$. Define for $w \in W$ the element $\beta(w)=$ $\tau_{w} \circ \lambda_{w} \in \operatorname{AGL}(\mathcal{U})$, where $\lambda_{w} \in \operatorname{GL}(\mathcal{U})$ is defined by $(v, z) \lambda_{w}=(v, z+e(v, w))$. If $w=\left(w_{1}, w_{2}, 0,0\right)$, then

$\beta(w):(x, y, 0,1, e \operatorname{Tr}(x)+b) \mapsto\left(x+w_{1}, y+w_{2}, 0,1, e \operatorname{Tr}(x)+b+e \operatorname{Tr}\left(w_{1}\right)\right)$.

So $\beta(w)$ leaves the set $\left\{(v, f(v)) \in \Gamma(f) \mid v \in W+v_{4}\right\}$ invariant. Also the set $\{(w, f(w)) \in \Gamma(f) \mid w \in W\}$ is invariant under $\beta(w)$. Obviously, the group $\mathcal{Q}$ centralizes $\beta(W)$ and a computation shows that $H$ normalizes $\beta(W)$. We deduce that $\Gamma(f)$ is invariant under $\beta(W)$, as $\Gamma(f)-\{(w, f(w)) \in \Gamma(f) \mid v \in W\}$ is the same as $\left\{(v, f(v)) \in \Gamma(f) \mid v \in W+v_{4}\right\} H \mathcal{Q}$. We remark that by Lemma $2.2 \beta(w)$ has to agree with the transformation defined by Equation (1) of Remark 2.5.

Proposition 3.9 Let $f=Q^{\sigma}$ be special parabolic as in Example 3.8. Then, $f$ is not a quadratic form.

Proof Assume that $f$ is a quadratic form. Define $W$ and $\widehat{W}$ as in Example 3.8. Since $f(0)=Q(0)=0$ and as $f$ vanishes on $W$, both $f$ and $Q$ are of hyperbolic type. By Witt's theorem [14, Thm. 12.10], there exist $\Phi \in \mathrm{GL}(V)$, such that $f(v)=Q(v \Phi)$ for $v \in V$ and as $Q$ and $f$ vanish on $W$, we can even assume that $\Phi_{W}=1_{W}$. Write $v \in V$ as $v=w+\widehat{w}$, with $w \in W, \widehat{w} \in \widehat{W}$. In particular, $\widehat{w} \Phi=\widehat{w} \Phi_{21}+\widehat{w} \Phi_{0}$ with $\Phi_{21} \in \operatorname{Hom}(\widehat{W}, W)$ and $\Phi_{0} \in \operatorname{GL}(\widehat{W})$. So $f(v)=Q(v \Phi)=\left(w+\widehat{w} \Phi_{21}, \widehat{w} \Phi_{0}\right)$. Here, $(\cdot, \cdot)$ is the bilinear form associated with $Q$. Denote by $[\cdot, \cdot]$ the bilinear form associated with $f$. Then:

$$
\left[w_{1}+\widehat{w}_{1}, w_{2}+\widehat{w}_{2}\right]=\left(w_{1}+\widehat{w}_{1} \Phi_{21}, \widehat{w}_{2} \Phi_{0}\right)+\left(w_{2}+\widehat{w}_{2} \Phi_{21}, \widehat{w}_{1} \Phi_{0}\right)
$$

Set $H_{0}=\left\langle h(v), w(1), \Theta \mid v \in F^{*}\right\rangle$. This group leaves the decomposition $V=$ $W \oplus \widehat{W}$ invariant. We identify $W$ and $\widehat{W}$ with $F^{2}$ in the obvious way. Then, $H_{0}$ has on both spaces the same representation (i.e., $\Phi_{W}=\Phi_{\widehat{W}}$ for $\Phi \in H_{0}$ ). We claim that this representation is absolutely irreducible: The subgroup $h\left(F^{*}\right)$ induces on $F \times 0$ 
and $0 \times F$ a Singer group, and we can assume that this Singer group induces the representations $D^{1}$ and $D^{-1}$, i.e., the $\mathbb{F}_{3} h\left(F^{*}\right)$-modules $F \times 0$ and $0 \times F$ are simple and non-equivalent. Also $h\left(F^{*}\right)\langle\Theta\rangle$ induces the normalizer of a Singer group, so that both modules are absolutely irreducible under this group (cf. Lemma 2.12 and [1, (25.8)]). Finally, $w(1)$ interchanges both modules showing the claim.

The action of $H_{0}$ will help us to pin down the concrete form of $\Phi$. For $h \in H_{0}$, we write $(w+\widehat{w}) h=w h_{W}+\widehat{w} h_{\widehat{W}}$, where $h_{W}$ is the restriction of $h$ to $W$ and $h_{\widehat{W}}$ the restriction of $h$ to $\widehat{W}$. Using Eq. (5), we have $\left(w h_{W}, \widehat{w} \Phi_{0} h_{\widehat{W}}\right)=\left(w, \widehat{w} \Phi_{0}\right)=$ $[w, \widehat{w}]=\left[w h_{W}, \widehat{w} h_{\widehat{W}}\right]=\left(w h_{W}, \widehat{w} h_{\widehat{W}} \Phi_{0}\right)$. We deduce $h_{\widehat{W}} \Phi_{0}=\Phi_{0} h_{\widehat{W}}$ and as $H_{0}$ induces an absolutely irreducible representation on $\widehat{W}$ we get $\Phi_{0}=c 1_{\widehat{W}}, c \in \mathbb{F}_{3}^{*}$. Replacing $f$ by $c^{-1} f$, we can assume even $c=1$, i.e., $\Phi_{0}=1$. Moreover, Eq. (5) becomes

$$
\left[w_{1}+\widehat{w}_{1}, w_{2}+\widehat{w}_{2}\right]=\left(\widehat{w}_{1}, w_{2}\right)+\left(w_{1}, \widehat{w}_{2}\right)+\left(\widehat{w}_{1}, \widehat{w}_{2} \Psi\right)
$$

where $\Psi=\Phi_{21}+\Phi_{21}^{*}$. We even may assume $\Phi_{21}=\frac{1}{2} \Psi=-\Psi$, since $\Psi^{*}=\Psi$ and $\left(\widehat{w}_{1}, \widehat{w}_{2} \Phi_{21}\right)+\left(\widehat{w}_{1} \Phi_{21}, \widehat{w}_{2}\right)=\left(\widehat{w}_{1}, \widehat{w}_{2} \Psi\right)=\left(\widehat{w}_{1}, \widehat{w}_{2} \frac{1}{2} \Psi\right)+\left(\widehat{w}_{1} \frac{1}{2} \Psi, \widehat{w}_{2}\right)$. Then

$\left(\widehat{w}_{1} h_{\widehat{W}}, \widehat{w}_{2} \Psi h_{W}\right)=\left(\widehat{w}_{1}, \widehat{w}_{2} \Psi\right)=\left[\widehat{w}_{1}, \widehat{w}_{2}\right]=\left[\widehat{w}_{1} h_{\widehat{W}}, \widehat{w}_{2} h_{\widehat{W}}\right]=\left(\widehat{w}_{1} h_{\widehat{W}}, \widehat{w}_{2} h_{\widehat{W}} \Psi\right)$.

Thus, $\Psi h_{W}=h_{\widehat{W}} \Psi$. So $\Psi$ can be identified with $c_{1} 1_{F^{2}}, c_{1} \in \mathbb{F}_{3}$ as $H_{0}$ is absolutely irreducible (on $F \times F$ ) and then $\Phi_{21}=-c_{1} 1_{F^{2}}$. But then $Q$ vanishes on the set $N \Phi^{-1}$, $N=\left\{\left(u^{2}+k,-u, u, 1\right) \mid u \in F\right\} \subseteq f^{-1}(0)$ (here we assume $\left.f(k, 0,0,1)=0\right)$. But $N \Phi^{-1}=\left\{\left(u^{2}+c_{1} u+k, c_{1}-u, u, 1\right) \mid u \in F\right\}$, showing $N \Phi^{-1} \nsubseteq Q^{-1}(0)$.

By [2], one knows that $\operatorname{Ext}_{\mathbb{F}_{5} \operatorname{SL}(2,5)}(M, M) \neq 0$, for $M=V(2,5)$ the natural $\mathbb{F}_{5} \operatorname{SL}(2,5)$-module. This means that there are indecomposable four-dimensional $\mathbb{F}_{5} \operatorname{SL}(2,5)$-modules with two composition factors $M$. This gives rise to further bent functions:

Example 3.10 Let $V=V(4,5)$. We define in $\operatorname{GL}(V)$ the elements $h=\operatorname{diag}(3,2,3,2)$ $\in \mathrm{GL}(V), w_{1}=\operatorname{diag}(J, J), J=\left(\begin{array}{ll}0 & 4 \\ 1 & 0\end{array}\right)$

$$
x_{+}=\left(\begin{array}{llll}
1 & 1 & 0 & 0 \\
0 & 1 & 0 & 0 \\
4 & 3 & 1 & 1 \\
3 & 4 & 0 & 1
\end{array}\right)
$$

and set $H=\left\langle h, w_{1}, x_{+}\right\rangle$. A computer calculation with MAGMA [3] shows $H \simeq$ $\operatorname{SL}(2,5)$. Set $W=\mathbb{F}_{5} \times \mathbb{F}_{5} \times 0 \times 0$. Then, $W$ is $H$-invariant and $V-W$ splits into five orbits $B_{0}, \ldots, B_{4}$ under $H$, each of it has length 120 . In particular, $V$ is an indecomposable $\mathbb{F}_{5} H$-module.

For any permutation $\sigma$ on $\{0, \ldots, 4\}$, define $f_{\sigma}: V \rightarrow \mathbb{F}_{5}$ by $f_{\sigma}(v)=0$ if $v \in W$ and $f_{\sigma}(v)=i$ if $v \in B_{i \sigma} \cap(V-W)$. Computing the Walch coefficients with MAGMA (it is enough to compute the Walch coefficients for representatives of $H$-orbits) shows 
that $f_{\sigma}$ is a 5-ary bent function. Again using MAGMA, one can compute the CCZ automorphism group and thus $\mathbf{E A}\left(f_{\sigma}\right)$. A procedure for this calculation is described in [12]. One gets $\mathbf{E A}\left(f_{\sigma}\right)=\mathbf{A}\left(f_{\sigma}\right) \leq \operatorname{GL}(\mathcal{U}), \mathcal{U}=V \times \mathbb{F}_{5}$ and $\mathbf{A}\left(f_{\sigma}\right) \simeq \operatorname{GL}(2,5)(40$ times) or $\mathbf{A}\left(f_{\sigma}\right)$ is isomorphic to $H C$ with $C \simeq \mathrm{C}_{4}, H \cap C=Z(H)$ and $[H, C]=1$ (80 times).

Remark 3.11 The construction of this example resembles closely the construction of the previous example. Nevertheless, $f_{\sigma}$ is not EA equivalent to a bent function $f$ of parabolic type: the 5-radical of $\mathbf{A}\left(f_{\sigma}\right)$ and that of $\mathbf{E}\left(f_{\sigma}\right)$ are trivial in contrast to $O_{p}(\mathbf{A}(f)) \neq 1, \mathbf{E}(f) \neq 1$.

Remark 3.12 Let $f$ be standard parabolic, twisted parabolic, or special parabolic with respect to the subspace $W$ of the quadratic space $V$. We have shown in Examples 3.4, 3.6 and 3.8 that $\mathbf{A}(f) \cap \operatorname{GL}(\mathcal{U})$ contains a subgroup, such that $W$ is the unique maximal subspace of $V$ invariant under this group.

\subsection{Maiorana functions with large cyclic automorphism groups}

Example 3.13 Let $n$ be a positive integer, $p$ a prime, $F=\mathbb{F}_{p^{n}}$ and $0<\ell<p^{n}-1$ a number coprime to $p^{n}-1$. Let $\operatorname{Tr}$ be the absolute trace on $F$. Set $V=F \times F$ and define $\mu_{\ell}: V \rightarrow \mathbb{F}_{p}$ by

$$
\mu_{\ell}(x, y)=\operatorname{Tr}\left(x y^{\ell}\right)
$$

Clearly, $\mu_{\ell}$ is a bent function of Maiorana type and this function is even a quadratic form, if $\ell$ is a $p$-power.

Let $\bar{\ell}$ be the inverse of $\ell$ modulo $p^{n}-1$ and define for $a \in F^{*}$ the operator $z_{a}=$ $\operatorname{diag}\left(T_{0}(a), T_{0}\left(a^{-\bar{\ell}}\right), 1\right)$. Then, $z_{a}$ lies $\mathbf{A}\left(\mu_{\ell}\right)$, since $\mu_{\ell}\left((x, y) z_{a}\right)=\mu_{\ell}(x, y)$, i.e., $\mathcal{Z}=$ $\left\{z_{a} \mid a \in F^{*}\right\}$ is a cyclic subgroup of $\mathbf{A}\left(\mu_{\ell}\right)$ of order $p^{n}-1$. As $\mu_{\ell}\left(x^{p}, y^{p}\right)=\mu_{\ell}(x, y)$, we see that $\Theta=\operatorname{diag}\left(T_{1}(1), T_{1}(1), 1\right)$ of order $n$ lies in $\mathbf{A}\left(\mu_{\ell}\right)$ and normalizes $\mathcal{Z}$. For $u \in \mathbb{F}_{p}^{*}$, we have $\mu_{\ell}(u x, y)=u \mu_{\ell}(x, y)$, so that $\pi_{u}=\operatorname{diag}\left(T_{0}(u), 1_{F}, u\right)$ lies in $\mathbf{A}\left(\mu_{\ell}\right)$ and centralizes $\mathcal{Z}$. Set $\mathcal{P}=\left\{\pi_{u} \mid u \in \mathbb{F}_{p}^{*}\right\}$. Then, $\mathcal{Z} \mathcal{P}\langle\Theta\rangle \simeq\left(\mathrm{C}_{p^{n}-1} \times\right.$ $\left.\mathrm{C}_{p-1}\right) \mathrm{C}_{n}$.

\section{Automorphism groups and equivalence of parabolic bent functions}

In this section, we consider parabolic $p$-ary bent functions $f: V=V(n, p) \rightarrow \mathbb{F}_{p}$, which are standard parabolic, twisted parabolic or special parabolic. For the descrip-

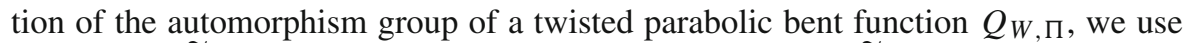
an operator $\widetilde{\Pi}$, which is derived from $\Pi$. The definition of $\widetilde{\Pi}$ can be found before Lemma 4.8. We set

$$
C_{\mathrm{GO}(V, Q)}^{*}(\widetilde{\Pi})=\left\{\Phi \in \mathrm{GO}(V, Q) \mid r_{\Phi}^{2} \widetilde{\Pi} \Phi=\Phi \widetilde{\Pi}\right\}
$$


We extend $\Pi$ to $V$ by setting $x \Pi=x$ for $x \in W_{1} \oplus W_{2}$. It will be clear from the context, when we consider $\Pi$ as an operator on $W_{0}$ or on $V$. The spaces $W_{0}, W_{1}, W_{2}$ have the meaning as in Example 3.6.

Theorem 4.1 Let $p>2$ be a prime, $V$ a finite-dimensional $\mathbb{F}_{p}$-space, $Q$ a nondegenerate quadratic form on $V$ and $f$ a proper parabolic bent function on $V$ with respect to $W$. Then, $\mathbf{E A}(f)=\mathbf{E}(f) \mathbf{A}(f)$ and $\mathbf{A}(f) \leq \mathrm{GL}(\mathcal{U}), \mathcal{U}=V \times \mathbb{F}_{p}$ the ambient space of $f$. Moreover:

(a) Let $f=Q_{W, a}^{b}$ be a standard parabolic bent function. Then, $\mathbf{E}(f)=\beta(W)$ if $b=1$, while $\mathbf{E}(f)=\beta(\operatorname{rad}(W))$ otherwise. Also $\mathbf{A}(f)=\mathrm{GO}(V, Q)_{W}$ if $a=0$ and $\mathbf{A}(f)=\mathrm{O}(V, Q)_{W}$ otherwise.

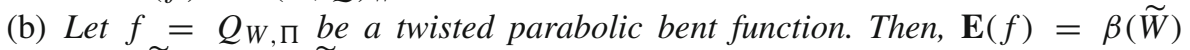
with $\left.\widetilde{W}=C_{W}(\widetilde{\Pi})\right) \cap\{w \in W \mid Q(w)=Q(w \Pi)\}$ and $\mathbf{A}(f)=L \mathcal{Q}$, $\mathcal{Q} \simeq O_{p}\left(\mathrm{GO}(V, Q)_{W}\right)$, where $L=\mathrm{GO}(V, Q)_{W_{0} W_{1}, W_{2}} \cap C_{\mathrm{GO}(V, Q)}^{*}(\widetilde{\Pi})$.

(c) Let $f=Q^{\sigma}$ be a special parabolic bent function on $V=V(4 n, 3)$. Then, $\mathbf{E}(f)=$ $\beta(W)$. The group $\mathbf{A}(f)$ has a normal subgroup $H \mathcal{Q}$ with $\mathcal{Q} \simeq O_{p}\left(\mathrm{O}(V, Q)_{W}\right)$ and $H \simeq \operatorname{SL}\left(2,3^{n}\right)$. Also $\mathbf{A}(f) / H \mathcal{Q} \simeq \mathrm{C}_{4} \cdot \operatorname{Aut}\left(\mathbb{F}_{3^{n}}\right)$ if $n$ is even and $\sigma$ fixes 0 and otherwise $\mathbf{A}(f) / H \mathcal{Q} \simeq \mathrm{C}_{2} \cdot \operatorname{Aut}\left(\mathbb{F}_{3^{n}}\right)$.

The answer to the equivalence problem is given by the next two corollaries.

Corollary 4.2 Let $p>2$ be a prime and $V$ a finite-dimensional $\mathbb{F}_{p}$-space. For $1 \leq$ $i \leq 2$, assume that $Q_{i}$ is a non-degenerate quadratic form on $V$ and that $f_{i}$ is a proper parabolic bent function (standard, twisted or special) with respect to $Q_{i}$ and the subspace $W^{i}$. Let $f_{1}$ and $f_{2}$ be EA equivalent. Then:

(a) $f_{1}$ and $f_{2}$ are linear equivalent.

(b) $Q_{1}$ and $Q_{2}$ have the same type.

(c) $W^{1}$ and $W^{2}$ are isometric.

(d) Either both $f_{1}$ and $f_{2}$ are standard or twisted parabolic or both $f_{1}$ and $f_{2}$ are special parabolic.

By the preceding corollary, we can assume for two EA equivalent parabolic bent functions that both are associated with the same quadratic form and the same subspace $W$.

Corollary 4.3 Let $p>2$ be a prime, $V$ a finite-dimensional $\mathbb{F}_{p}$-space, $Q$ a nondegenerate quadratic form on $V$ and let $f$ and $f^{\prime}$ be a proper parabolic bent function (standard, twisted or special) with respect to the subspace $W$.

(a) Let $f=Q_{W, a}^{b}$ and $f^{\prime}=Q_{W, a^{\prime}}^{b^{\prime}}$. Then, $f$ is EA equivalent to $f^{\prime}$, iff $b=b^{\prime}$ and if either $\operatorname{dim} V$ is even and $a \mathbb{F}_{p}^{*}=a^{\prime} \mathbb{F}_{p}^{*}$ or if $\operatorname{dim} V$ is odd and $a\left(\mathbb{F}_{p}^{*}\right)^{2}=a^{\prime}\left(\mathbb{F}_{p}^{*}\right)^{2}$.

(b) Let $f=Q_{W, \Pi}$ and $f^{\prime}=Q_{W, \Pi^{\prime}}$ be twisted parabolic bent functions. Then, $f$ is EA equivalent to $f^{\prime}$ iff there exist $\Phi_{1}, \Phi_{2} \in \mathrm{GO}\left(W_{0}, Q\right)$, such that $\Phi_{1}$ has the same multiplier as $\Phi_{2}$ and $\Phi_{1} \Pi=\Pi^{\prime} \Phi_{2}$.

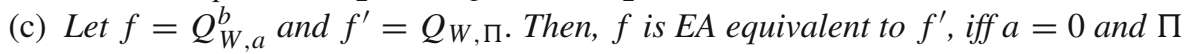
lies $\mathrm{GO}\left(W_{0}, Q_{W_{0}}\right)$ and has multiplier $b^{-1}$.

(d) Let $f=Q^{\sigma}$ and $f^{\prime}=Q^{\sigma^{\prime}}$ be special parabolic on $V=V(4 n, 3)$. Then, $f$ is $E A$ equivalent to $f^{\prime}$ iff either $n$ is even and $\sigma^{\prime} \in\langle(2,3)\rangle \sigma\langle(2,3)\rangle$ or if $n$ is odd and $\sigma^{\prime} \in\langle(2,3)\rangle \sigma$. 


\subsection{Derivatives of parabolic bent functions}

Lemma 4.4 Let $p>2$ be a prime, $V=V(n, p), n \geq 2$ and $Q$ a non-degenerate quadratic form on $V$. Let $f: V \rightarrow \mathbb{F}_{p}$ be a standard parabolic or twisted parabolic bent function with respect to the subspace $W$ of $V$.

(a) Let $f=Q_{W, a}^{b}$ a proper standard parabolic bent function. Let $0 \neq v \in V$. Then, $D_{v}^{2} f=0$ iff $v \in W$ and $Q(v)=0$.

(b) Let $f=Q_{W, \Pi}$ be a proper twisted parabolic bent function. Let $0 \neq v \in V$. Then, $D_{v}^{2} f=0$ iff $v \in W$ and $Q(v)=Q(v \Pi)=0$.

Proof (a) Let $(\cdot, \cdot)$ be the bilinear form associated with $Q$. Set $f=Q_{W, a}^{b}$ and assume first $v \in W$. For $x \in V$ either both $x, x+v \in W$ or both $x, x+v \notin W$. Hence, $D_{v} f(x)=Q(v)+(v, x)$ if $x \in W$ and $D_{v} f(x)=b(Q(v)+(v, x))$ otherwise. Thus, by Lemma $2.10 D_{v}^{2} f(x)=2 Q(v)$ if $x \in W$ and $D_{v}^{2} f(x)=2 b Q(v)$ otherwise and $D_{v}^{2} f(x)=0$ iff $Q(v)=0$.

Assume now $v \notin W$. If $x \in W$, then $x+v, x+2 v \notin W$. Moreover

$$
D_{v} f(x)=b Q(x+v)+a-Q(x)=(b-1) Q(x)+b Q(v)+b(v, x)+a .
$$

So

$$
\begin{aligned}
D_{v}^{2} f(x)= & D_{2 v} f(x)-2 D_{v} f(x) \\
= & (b-1) Q(x)+b Q(2 v)+b(2 v, x)+a \\
& -2((b-1) Q(x)+b Q(v)+b(v, x)+a) \\
= & -(b-1) Q(x)+2 b Q(v)-a .
\end{aligned}
$$

Then, $D_{v}^{2} f \neq 0$, if $2 b Q(v) \neq a$. So assume $2 b Q(v)=a$. If $W^{\perp}$ is a proper subspace of $W$ and $b \neq 1$, the function $(b-1) Q$ takes at least two values on $W$ and we conclude $D_{v}^{2} f \neq 0$. So we assume $W=W^{\perp}$ (and then $b=1$ and $a \neq 0$ by our assumptions) or we assume $W \neq W^{\perp}$ and $b=1$.

We claim that we can pick $x \notin W$, such that $x+v, x+2 v \notin W$ : This is obvious if $W+\langle v\rangle$ is a proper subspace of $V$ or if $V=W+\langle v\rangle$ and $p>3$. So assume $V=W+\langle v\rangle$ and $p=3$. Then, $f$ is a quadratic form by Remark 3.5, contradicting the fact that $f$ is proper parabolic. Our claim holds.

Hence, $D_{w} f(x)=b(Q(w)+(w, x))$ for $w=v, 2 v$ and $D_{v}^{2} f(x)=b(Q(2 v)+$ $(2 v, x)-2(Q(v)+(v, x))=2 b Q(v)$. So $D_{v}^{2} f \neq 0$ if $Q(v) \neq 0$. So we assume $Q(v)=0$. But this forces $a=0$, ruling out the case $W=W^{\perp}$. So $W \neq W^{\perp}, b=1$ and $Q(v)=a=0$, which was not allowed too.

(b) We can write

$$
Q_{W, \Pi}(x)= \begin{cases}Q(x \Pi), & x \in W, \\ Q(x), & x \notin W .\end{cases}
$$

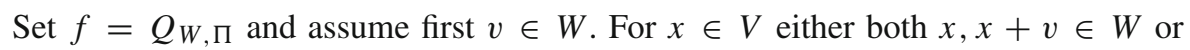
both $x, x+v \notin W$. Hence, $D_{v} f(x)=Q(v \Pi)+(v \Pi, x \Pi)$ if $x \in W$ and $D_{v} f(x)=$ 
$Q(v)+(v, x)$ otherwise. Thus, by Lemma $2.10 D_{v}^{2} f(x)=2 Q(v \Pi)$ if $x \in W$ and $D_{v}^{2} f(x)=2 Q(v)$ otherwise and $D_{v}^{2} f=0$ iff $Q(v)=Q(v \Pi)=0$.

Assume now $v \notin W$. If $x \in W$, then $x+v, x+2 v \notin W$. Moreover,

$$
D_{v} f(x)=Q(x+v)-Q(x \Pi)=Q(x)+Q(v)+(v, x)-Q(x \Pi) .
$$

So

$$
\begin{aligned}
D_{v}^{2} f(x)= & D_{2 v} f(x)-2 D_{v} f(x) \\
= & Q(x)+Q(2 v)+(2 v, x)-Q(x \Pi) \\
& -2(Q(x)+Q(v)+(v, x)-Q(x \Pi)) \\
= & Q(x \Pi)-Q(x)+2 Q(v) .
\end{aligned}
$$

As the function $Q(x \Pi)-Q(x)$ takes at least two values on $W_{0}$, we conclude $D_{v}^{2} f \neq 0$.

Lemma 4.5 Let $f=Q^{\sigma}$ be a special parabolic bent function on $V=V(4 n, 3), n \geq 2$ with respect to the subspace $W$. Let $v \in V$. Then, $D_{v}^{2} f=0$ iff $v \in W$.

Proof We assume as in Example 3.8 that $V=F \times F \times F \times F, W=F \times F \times 0 \times 0$ and $\widehat{W}=0 \times 0 \times F \times F$. We know that $f$ is a function of Maiorana type with

$$
f(w+\widehat{w})=\left(w, \widehat{w}^{\pi}\right),
$$

where $w \in W, \widehat{w} \in \widehat{W}$ and $\pi$ is a permutation on $\widehat{W}$.

Let $v \in W$. Then, $D_{v} f(w+\widehat{w})=\left(w+v, \widehat{w}^{\pi}\right)-\left(w, \widehat{w}^{\pi}\right)=\left(v, \widehat{w}^{\pi}\right)$ and

$$
D_{v}^{2} f(w+\widehat{w})=\left(2 v, \widehat{w}^{\pi}\right)-2\left(v, \widehat{w}^{\pi}\right)=0 .
$$

Let $v \in V-W$. By Remark 2.11, it is enough to show $D_{v}^{2} f \neq 0$ for representatives from the orbits $B_{i}, 0 \leq i \leq 2$. Let $v=v_{4}=(0,0,0,1)$.

Assume first $0^{\sigma} \neq 0$. Pick $k \in F^{*}$, such that $\operatorname{Tr}(k)^{\sigma}=0$. Then

$$
D_{v} f(k, 0,0,0)=\operatorname{Tr}(k)^{\sigma}-0=0
$$

and $D_{2 v} f(k, 0,0,0)=\operatorname{Tr}(-k)^{\sigma}-0 \neq 0$. Hence $D_{v}^{2} f(k, 0,0,0) \neq 0$, i.e., $D_{v}^{2} f \neq 0$.

Assume now $0^{\sigma}=0$. Since $Q^{\sigma}$ is equivalent to $Q^{i d}$, we may assume that $\sigma$ is the trivial permutation. Pick $a \in F$, such that $\operatorname{Tr}(a)=1$ and set $x=(a, 0,0,1)$. Then, $D_{v} f(x)=f(a, 0,0,2)-f(a, 0,0,1)=-\operatorname{Tr}(a)-\operatorname{Tr}(a)=\operatorname{Tr}(a)$ and $D_{2 v} f(x)=f(a, 0,0,0)-f(a, 0,0,1)=-\operatorname{Tr}(a)$. Hence, $D_{v}^{2} f(x)=D_{2 v} f(x)-$ $2 D_{v} f(x)=-\operatorname{Tr}(a)-\operatorname{Tr}(a)=1 \neq 0$, i.e., $D_{v}^{2} f \neq 0$. In a similar fashion, one shows $D_{v}^{2} f \neq 0$, if $v$ has the shape $(b, 0,0,1), b \neq 0$.

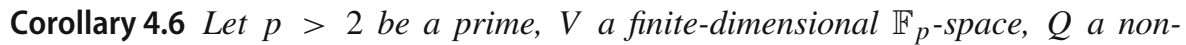
degenerate quadratic form on $V, W$ a subspace and $f$ a proper parabolic bent function with respect to $W$. Let $\Phi \in \mathbf{A}(f) \cap \mathrm{GL}(\mathcal{U}), \mathcal{U}=V \times \mathbb{F}_{p}$. Then, $\Phi$ fixes the spaces $W$ and $\operatorname{rad}(W)$. 
Proof The assertion follows from Lemmas 4.4 and 4.5, if $W=\operatorname{rad}(W)$. So assume

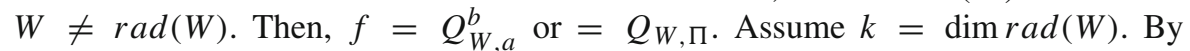
Lemma $4.4, \operatorname{rad}(W)$ is the only $k$-flat on which the double derivative vanishes.

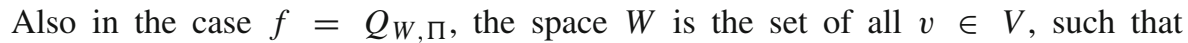
$f(x+v)-f(x)-f(v)=0$ for all $x \in \operatorname{rad}(W)$. The assertion follows by Remark 2.11.

\subsection{The groups $A(f)$}

Let $f: V=V(n, p) \rightarrow \mathbb{F}_{p}$ be a bent function of parabolic type. In this subsection, we set $\mathcal{U}=V \times \mathbb{F}_{p}, \mathcal{U}_{0}=0 \times \mathbb{F}_{p}$ and identify $V$ in the obvious way with $V \times 0$. By Lemma 2.3, $\mathbf{A}(f) \leq \operatorname{GL}(\mathcal{U})$ and this group fixes the decomposition $\mathcal{U}=V \oplus \mathcal{U}_{0}$.

Lemma 4.7 Let $p>2$ be a prime, $V=V(n, p), n \geq 2$ and $Q$ a non-degenerate quadratic form on $V$. Let $f=Q_{W, a}^{b}$ be a proper standard parabolic bent function with respect to the subspace $W$ of $V$. Then, $\mathbf{A}(f)=\mathrm{GO}(V, Q)_{W}$ if $a=0$ and $\mathbf{A}(f)=\mathrm{O}(V, Q)_{W}$ otherwise.

Proof Let $\Phi \in \mathbf{A}(f)$. Then, for all $x \in V$, we have

$$
f(x \Phi)=c f(x),
$$

where $c$ is the scalar induced by $\Phi$ on $0 \times \mathbb{F}_{p}$. By Corollary 4.6, $\Phi$ fixes $W$ and $\operatorname{rad}(W)$.

Let $x \in W$. Then, $Q(x \Phi)=c Q(x)$. If $x \notin W$, then $b Q(x \Phi)+a=c(b Q(x)+a)$ or

$$
Q(x \Phi)=c Q(x)+C, \quad C=b^{-1} a(c-1) .
$$

If $a=0$, the assertion of the lemma already follows. So assume $a \neq 0$. Replace $x$ by $2 x$ and $4 Q(x \Phi)=4 c Q(x)+C$ follows. Hence, $C=0$, i.e., $c=1$ if $p>3$. So assume $p=3$. Then, $\operatorname{dim} V / W \geq 2$. So there exist $x, y \notin W$ with $x+\varepsilon y \notin W$, $\varepsilon= \pm 1$. This leads to

$$
Q(x \Phi)+Q(y \Phi)+\varepsilon(x \Phi, y \Phi)=c(Q(x)+Q(y)+\varepsilon(x, y))+C
$$

So $Q(x \Phi)+Q(y \Phi)=c(Q(x)+Q(y))+C$ showing again $C=0$, i.e., $c=1$. The proof is complete.

Let $V, W, W_{0}, W_{1}$ and $\Pi$ be as in Example 3.6. Extending $\Pi$ to a transformation in $\mathrm{GL}(V)$ as above, we set

$$
\widetilde{\Pi}=\Pi \Pi^{*} \text {. }
$$

Lemma 4.8 Let $p>2$ be a prime, $V=V(n, p), n \geq 2$ and $Q$ a non-degenerate quadratic form on $V$. Let $f=Q_{W, \Pi}$ be a proper twisted parabolic bent function 
with respect to the subspace $W$ of $V$. Then, $\mathbf{A}(f)=L \mathcal{Q}, \mathcal{Q} \simeq O_{p}\left(\mathrm{GO}(V, Q)_{W}\right)$ and $L=\mathrm{GO}(V, Q)_{W, W_{0}, W_{1}} \cap C_{\mathrm{GO}(V, Q)}^{*}(\widetilde{\Pi})$.

Proof By Corollary 4.6, $\Phi \in G=\mathbf{A}(f)$ fixes $W$ and $\operatorname{rad}(W)$. Let $\mathcal{Q}$ be the normal subgroup of operators of $G$, whose restrictions to $\operatorname{rad}(W), W / \operatorname{rad}(W)$ and $V / W$ are in each case the identity. Then, the restriction of such an operator to $V$ is unipotent, i.e., the order of $\Phi$ is a $p$-power, as its restriction to $V$ is faithful. The restriction to $\mathcal{U}_{0}$ lies in $\operatorname{GL}\left(\mathcal{U}_{0}\right) \simeq \mathbb{F}_{p}^{*}$. Hence, the restriction to $\mathcal{U}_{0}$ is the identity, i.e.,

$$
f(x \Phi)=f(x) \text { for } x \in V \text { and } \Phi \in \mathcal{Q} .
$$

So $Q(x \Phi)=Q(x)$ for $x \notin W$ and $Q(x \Phi \Pi)=Q(x \Pi)$ for $x \in W$. But for $x \in W$, we have by definition of $\Phi$ the equation $Q(x \Pi)=Q(x \Pi \Phi)$. So $\Phi \in \mathrm{GO}(V, Q)$ and by Corollary 3.3 we obtain $\mathcal{Q} \simeq O_{p}\left(\mathrm{GO}(V, Q)_{W}\right)$.

Let $\tau_{1} \in \mathrm{GL}(\mathcal{U})$ be the identity on $W_{0}$ and $\mathcal{U}_{0}$ and let $\tau_{1}$ induce -1 on $W_{1} \oplus W_{2}$. Let $\tau_{2} \in \mathrm{GL}(\mathcal{U})$ be the identity on $W_{1} \oplus W_{2}$ and $\mathcal{U}_{0}$, and let $\tau_{2}$ induce -1 on $W_{0}$. Then, $\mathcal{T}=\left\langle\tau_{1}, \tau_{2}\right\rangle$ is a four group in $G$, such that

$$
\mathcal{T} \mathcal{Q} / \mathcal{Q} \leq Z(G / \mathcal{Q})
$$

since $G$ fixes $W, W_{1}$ and $V$. Moreover, $\mathcal{T}$ is a Sylow 2-subgroup of $\mathcal{T} \mathcal{Q}$. A similar Frattini argument as in the proof of Lemma 2.3 leads to

$$
G=C_{G}(\mathcal{T}) \mathcal{Q}
$$

Let $\Phi \in C_{G}(\mathcal{T})$. As $C_{V}\left(\tau_{1}\right)=W_{0}$ and $C_{V}\left(\tau_{2}\right)=W_{1} \oplus W_{2}$, the operator $\Phi$ leaves the decomposition $\mathcal{U}=W_{0} \oplus\left(W_{1} \oplus W_{2}\right) \oplus \mathcal{U}_{0}$ invariant. Suppose

$$
f(x \Phi)=c f(x), \quad x \in V .
$$

As the restriction of $f$ to $W_{1} \oplus W_{2}$ is $Q$, we see that the restriction of $\Phi$ to $W_{1} \oplus W_{2}$ is an element in $G O\left(W_{1} \oplus W_{2}, Q\right)$ with multiplier $c$. Let $0 \neq x_{2} \in W_{2}$. Then $Q(y \Phi)=Q\left(\left(x_{2}+y\right) \Phi\right)=f\left(\left(x_{2}+v\right) \Phi\right)=c f\left(x_{2}+y\right)=c Q(y)$ for all $y \in W_{0}$, since $Q\left(x_{2} \Phi\right)=Q\left(x_{2}\right)=0$ and $\left(x_{2} \Phi, y \Phi\right)=\left(x_{2}, y\right)=0$. Thus, $\Phi$ is an element in $\mathrm{GO}(V, Q)$ with multiplier $c$.

On the other hand, $Q(y \Phi \Pi)=f(y \Phi)=c f(y)=c Q(y \Pi)=Q(y \Pi \Phi)$. So for $y, y^{\prime} \in W_{0}$ and as $\Phi^{-1}$ has multiplier $c^{-1}$ :

$$
\begin{aligned}
c\left(y^{\prime}, y \Pi \Pi^{*}\right) & =c\left(y^{\prime} \Pi, y \Pi\right)=\left(y^{\prime} \Pi \Phi, y \Pi \Phi\right) \\
& =\left(y^{\prime} \Phi \Pi, y \Phi \Pi\right)=c^{-1}\left(y^{\prime}, y \Phi \Pi \Pi^{*} \Phi^{-1}\right)
\end{aligned}
$$

So $c^{2} \widetilde{\Pi}=\Phi \widetilde{\Pi} \Phi^{-1}$, and we conclude $C_{G}(\mathcal{T}) \leq \operatorname{GO}(V, Q)_{W, W_{0}, W_{1}} \cap C_{\mathrm{GO}(V, Q)}^{*}(\widetilde{\Pi})$. The verification of the reverse inclusion is routine.

Lemma 4.9 Let $f=Q^{\sigma}$ be a special parabolic 3-ary bent function on $V=V(4 n, 3)$, $n \geq 2$, and assume the notation of Example 3.8. Then, $N_{\mathbf{A}(f)}(\mathcal{Q} H)=\mathcal{Q} H\langle\Theta, z(v)\rangle$ if $n$ is even and $\sigma$ fixes 0 . Otherwise, $N_{\mathbf{A}(f)}(\mathcal{Q} H)=\mathcal{Q} H\langle\Theta, z(-1)\rangle$. 
Proof We denote by $L_{V}$ the restriction of a subgroup $L \leq \mathbf{A}(f)$ to $V$. Of course, this restriction map is faithful. First, we claim:

(*) $N_{\mathrm{GL}(V)}\left((\mathcal{Q} H)_{V}\right)=(\mathcal{Q} H\langle\Theta, z(\nu)\rangle)_{V}$ if $n$ is even and $=(\mathcal{Q} H\langle\Theta, z(-1)\rangle)_{V}$ if $n$ is odd.

By Dickson's theorem, every cyclic group of order $3^{n}-1$ in $(\mathcal{Q} H / \mathcal{Q})_{V} \simeq \operatorname{SL}(2, F)$ is conjugate to $\left(h\left(F^{*}\right) \mathcal{Q} / \mathcal{Q}\right)_{V}$ and so by the Schur-Zassenhaus theorem $h\left(F^{*}\right)_{V}$ represents the only conjugacy class of cyclic groups of order $3^{n}-1$ in $(\mathcal{Q} H)_{V}$. Then, $N_{\mathrm{GL}(V)}\left((\mathcal{Q} H)_{V}\right)=(\mathcal{Q} H)_{V} N_{\mathrm{GL}(V)}\left(h\left(F^{*}\right)_{V}\right)$ by a Frattini argument. Note that this normalizer leaves invariant $W=C_{V}\left(\mathcal{Q}_{V}\right)$. We even can write $N_{\mathrm{GL}(V)}\left((\mathcal{Q} H)_{V}\right)=$ $(\mathcal{Q} H\langle\Theta\rangle)_{V} C_{\mathrm{GL}(V)}\left(h\left(F^{*}\right)_{V}\right) \cap \mathrm{GL}(V)_{W}$, since $h\left(F^{*}\right)\langle\Theta\rangle$ can be considered as the normalizer of a Singer group of $\mathrm{GL}_{\mathbb{F}_{3}}(F)$. We have to determine $C_{\mathrm{GL}(V)}\left(h\left(F^{*}\right)_{V}\right) \cap$ $\mathrm{GL}(V)_{W}$. Let $\alpha$ be an element of this group.

(i) We may assume that $\alpha=\tau \delta$, with $\delta=\operatorname{diag}\left(T_{0}(u v), T_{0}(v), T_{0}(u), T_{0}(1)\right), u, v \in$ $F^{*}$ and

$$
\tau=\left(\begin{array}{cccc}
1 & 0 & 0 & 0 \\
0 & 1 & 0 & 0 \\
T_{0}\left(b_{1}\right) & 0 & 1 & 0 \\
0 & T_{0}\left(b_{2}\right) & 0 & 1
\end{array}\right)
$$

The element $\alpha$ fixes $W$ and the homogeneous components of $h\left(F^{*}\right)_{V}$, which are $F \times 0 \times F \times 0$ and $0 \times F \times 0 \times F$. Adjusting $\alpha$ by an element from $h\left(F^{*}\right)$, we may therefore assume that $\alpha_{V / W}=\operatorname{diag}\left(T_{0}(u), 1\right), u \in F^{*}$, which in turn forces $\alpha_{W}=\operatorname{diag}\left(T_{0}(u v), T_{0}(v)\right), v \in F^{*}$ : Note that $\alpha_{W}$ induces via conjugation on $(H \mathcal{Q} / \mathcal{Q})_{W}$ the same automorphism as $\alpha_{V / W}$ induces on $(H \mathcal{Q} / \mathcal{Q})_{V / W}$, i.e., these elements differ by an operator of the form $\operatorname{diag}\left(T_{0}(v), T_{0}(v)\right)$. So $\tau=\alpha \delta^{-1}$ acts trivially on $W$ and $V / W$ as well as the homogeneous components of $h\left(F^{*}\right)_{W}$. Since $\tau$ lies in the centralizer of $h\left(F^{*}\right)_{V}$, a simple calculation shows assertion (i).

(ii) $u v \in \mathbb{F}_{3}$.

Write $T \in \mathcal{Q}$ as in Example 3.8. Then, $T_{V}^{\alpha}=T_{V}^{\delta} \in \mathcal{Q}_{V}$, which implies

$$
T_{0}(u v) T((d)) T_{0}\left(u^{-1}\right)=\left(T_{0}\left(u^{-1}\right) T((a)) T_{0}(u v)\right)^{*}=T((d)) T_{0}(v),
$$

i.e., $T_{0}(u v)$ lies in the center of $\operatorname{End}_{\mathbb{F}_{3}}(F)$ and assertion (ii) follows.

(iii) $\tau \in \mathcal{Q}_{V}$ and $\delta \in\left\langle z(v)_{V}\right\rangle$.

A computation shows

$$
x_{+}(1)^{\alpha}=\left(\begin{array}{cccc}
1 & 0 & 0 & 0 \\
u & 1 & 0 & 0 \\
0 & 0 & 1 & 0 \\
T_{0}\left(\left(b_{2}-b_{1}\right) u v\right)+T_{1}(u v) & T_{1}(-v) & u & 1
\end{array}\right) .
$$


Then, $x_{+}(1)^{\alpha} x_{+}(-u) \in \mathcal{Q}_{V}$ has the form

$$
\left(\begin{array}{cccc}
1 & 0 & 0 & 0 \\
0 & 1 & 0 & 0 \\
0 & 0 & 1 & 0 \\
T_{0}\left(\left(b_{2}-b_{1}\right) u v\right)+T_{1}\left(u^{2}-u v\right) & T_{1}(u-v) & 0 & 1
\end{array}\right) .
$$

This forces $u=v$ and $b_{1}=b_{2}$. So $u^{2} \in \mathbb{F}_{3}$ by (ii), $\tau \in \mathcal{Q}_{V}$ and $\delta=\left\langle z( \pm v)_{V}\right\rangle$. Claim (iii) follows and thus Claim (*).

Clearly, $N_{\mathbf{A}(f)}(\mathcal{Q} H)_{V} \leq N_{\mathrm{GL}(V)}(\mathcal{Q} H)_{V}$. We already know that $z(-1) \in \mathbf{A}(f)$ and $z(v) \in \mathbf{A}(f)$ if $n$ is even and $\sigma$ fixes 0 . So the assertion of the lemma holds, if $n$ is odd or $n$ is even and $\sigma$ fixes 0 .

So consider the case that $\sigma$ moves $0, n$ is even and assume $z \in \mathbf{A}(f), z_{V}=z(v)_{V}$, and $z \mathcal{U}_{0}= \pm 1$. Set $N=f^{-1}(0) \cap\left(v_{4}+(F \times F \times F \times 0)\right)$. The action of $x_{+}(F) \mathcal{Q}$ shows that $N=\left\{\left(k+y+u^{2}, x-u, u, 1\right) \mid x, u \in F, y \in F_{0}\right\}$, where $F_{0}$ is the kernel of the trace map and $(k, 0,0,1)$ is a representative of the $x_{+}(F) \mathcal{Q}$-orbit $N$. Then, $\operatorname{Tr}(k) \neq 0$, as $f\left(v_{4}\right)=0$ iff $\sigma$ fixes 0 . $N$ is invariant under $z$, which shows that $(-k, 0,0,1) \in N$ or $-k \in k+F_{0}$ forcing $\operatorname{Tr}(k)=0$, a contradiction. The proof is complete.

Lemma 4.10 Let $f$ be a special parabolic 3-ary bent function on $V=V(4 n, 3), n \geq 2$ and assume the notation of Example 3.8. Then, $\mathcal{Q} H \unlhd \mathbf{A}(f)$.

Proof We know that $G=\mathbf{A}(f) \leq \mathrm{GL}(\mathcal{U})$ and that $W$ is $G$-invariant by Corollary 4.6.

We claim $O_{3}(G)=\mathcal{Q}$ : Let $\Phi \in O_{3}(G)$. Then, $C_{X}(\Phi) \neq 0$ for $X \in\left\{W, V / W, \mathcal{U}_{0}\right\}$ as $O_{3}(G)$ is a 3-group [1, (5.15)]. Also every such $X$ is an irreducible $H$-module, which forces $\Phi_{X}=1_{X}$. Therefore, for all $v \in V$, we have $f(v \Phi)=f(v)$ and $v \Phi-v \in W$. We have seen in the proof of the previous lemma that $f$ takes on $v_{4}+W$ every value of $\mathbb{F}_{3}$ precisely $|W| / 3$ times. So this fact holds too for any $v \in V-W$ (as $H$ acts transitively on the non-trivial cosets of $V / W$ ). Since $\mathcal{Q} \leq O_{3}(G)$ and $|[V, \mathcal{Q}]=| W \mid / 3$, we deduce from $f(v \Phi)=f(v)$ that $Q(v \Phi)=Q(v)$ holds too, hence our claim.

For $X=W$ or $=V / W$, let $N_{X}$ be the kernel of the action of $G$ on $X$. Of course, $N_{W} \cap N_{V / W}=\mathcal{Q}$.

(i) $N_{W}=N_{V / W}=\mathcal{Q}$.

The group $G / N_{X}$ acts transitively on $X-0$, and since $H N_{X} / N_{X} \simeq H \simeq \operatorname{SL}\left(2,3^{n}\right)$, we deduce from Hering's theorem [20] that $G$ has a normal subgroup $S$, such that

$$
S / N_{X} \simeq \operatorname{SL}\left(2 a, 3^{b}\right) \text { or } \operatorname{Sp}\left(2 a, 3^{b}\right), \quad n=a b
$$

and $H \simeq H N_{X} / N_{X} \leq S / N_{X}$.

If $\Phi \in N_{V / W}$ and $\Phi_{W}$ centralizes $H_{W}$, then $[H, \Phi] \leq N_{V / W}$ and $[H, \Phi]_{W}=1$ or $[H, \Phi] \leq N_{W} \cap N_{V / W}$. So $\Phi \in N_{G}(H \mathcal{Q})$. But $N_{V / W} \cap N_{G}(H \mathcal{Q})=\mathcal{Q}$ by Lemma 4.9. So only elements from $\mathcal{Q}$ are those elements in $N_{V / W}$, which centralize $H_{W}$. But any non-trivial normal subgroup of $G / N_{W}$ contains also non-trivial elements, 
which centralize $S / N_{W}$ and thus $H_{W}$. This forces $N_{V / W}=\mathcal{Q}$ and by symmetry claim (i) follows.

(ii) $G$ has a normal subgroup $S$, such that $S / \mathcal{Q} \simeq \operatorname{SL}\left(2 a, 3^{b}\right)$ or $\operatorname{Sp}\left(2 a, 3^{b}\right)$ for $n=a b$.

This follows from (i) and Eq. (6).

(iii) $a=1$.

Suppose $a>1$. Since $\operatorname{Sp}\left(2 a, 3^{b}\right) \leq \mathrm{SL}\left(2 a, 3^{b}\right)$, we can limit our attention to the case $\bar{S}=S / \mathcal{Q} \simeq \operatorname{Sp}\left(2 a, 3^{b}\right)$. Let $E=\left\{\Phi \in \mathrm{GL}(V) \mid \Phi_{W}=1_{W}, \Phi_{V / W}=1_{V / W}\right\}$. Then, $E$ becomes an $\mathbb{F}_{3} S$-module under conjugation by $S$. Clearly, $\mathcal{Q}$ lies in the kernel of this representation, so that we can and do consider $E$ as an $\mathbb{F}_{3} \bar{S}$-module. Let $L$ be the natural $\mathbb{F}_{3^{b}} \bar{S}$-module, and let $\phi$ be the Frobenius automorphism of $\mathbb{F}_{3^{b}}$. Denote by $L^{\phi^{i}}$ the $i$ th Frobenius twist of $L$. Then, by [2, p. 218] $E$ is the direct sum of the modules $L \otimes L^{\phi^{i}}, 0 \leq i<b$. Now we follow [11, Sect. 2] (the restriction there to even characteristic is not essential). The module $M_{i}=L \otimes L^{\phi^{i}}$ is simple even as an $\mathbb{F}_{3} \bar{S}$ module for $i>0$ and $i \neq \frac{b}{2}$ ( $b$ even). If $b$ is even, then $L \otimes L^{\phi^{b / 2}} \simeq M_{b / 2} \oplus M_{b / 2}$, $M_{b / 2}$ a simple $\mathbb{F}_{3} \bar{S}$-module. Set $\mathcal{A}=\wedge^{2}(L)$ and $\mathcal{S}=S^{2}(L)$ (second term of the symmetric algebra over $L$ ). Then, $\mathcal{S}$ is simple and $L \otimes L \simeq \mathcal{A} \oplus \mathcal{S}$. Finally, we have $L \otimes L^{\phi^{i}} \simeq L \otimes L^{\phi^{b-i}}$ as $\mathbb{F}_{3} \bar{S}$-modules and $M_{i} \simeq M_{j}$ for $i, j>0$ iff $j=i$ or $=b-i$.

Then, by [11, Remark 2.5], we have decompositions of $\mathbb{F}_{3} \bar{S}$-modules

$$
\mathcal{Q} \simeq \mathcal{A} \oplus \bigoplus_{i=1}^{\lfloor b / 2\rfloor} M_{i} \text { and } E / \mathcal{Q} \simeq \mathcal{S} \oplus \bigoplus_{i=1}^{\lfloor b / 2\rfloor} M_{i}
$$

Set $\widehat{W}=0 \times 0 \times F \times F$. For $g \in S$ and $\widehat{w} \in \widehat{W}$ write $\widehat{w} g=\widehat{w} B(g)+\widehat{w} g_{\widehat{W}}$ with $\widehat{w} B(g) \in W$ and $\widehat{w} g_{\widehat{W}} \in \widehat{W}$. Then, $B\left(g_{1} g_{2}\right)=\left(g_{1}\right)_{\widehat{W}} B\left(g_{2}\right)+B\left(g_{1}\right)\left(g_{2}\right)_{W}$, i.e., if we set $C(g)=g_{\widehat{W}}^{-1} B(g)$, then $C: \bar{S} \rightarrow \operatorname{End}_{\mathbb{F}_{3}}\left(F^{2}\right) \simeq E$ is a 1-cocycle of $\bar{S}$ into $E$ and $C$ induces a 1 -cocycle into $E / \mathcal{Q}$ too. The restriction of this cocycle to the subgroup to $H \mathcal{Q} / \mathcal{Q}$ is not a coboundary. Therefore, the restriction of $C$ to $\bar{S}$ is not a coboundary too. But $\mathrm{H}^{1}(\bar{S}, E / \mathcal{Q})=0$ by Theorem 2.15 (or Lemma 2.17 if $\bar{S} \simeq \operatorname{Sp}(4,3)$ ). So (iii) holds.

By (iii), we have $S=\mathcal{Q} H$, and the lemma follows

\subsection{The groups $E(f)$}

In this subsection, $p>2$ is an odd prime.

Lemma 4.11 Let $V$ be a finite-dimensional $\mathbb{F}_{p}$-space, $Q$ a non-degenerate quadratic form on $V$ and $f: V \rightarrow \mathbb{F}_{p}$ be a proper parabolic bent function with respect to the subspace $W$ of $V$. Let $\beta(v) \in \mathbf{E}(f)$. Then, $v \in W$.

Proof Let $M \subseteq V$ be the subspace with $\beta(M)=\mathbf{E}(f)$ (c.f. Remark 2.5). Assume $M \nsubseteq W$. We know from Remark 3.12 that $\mathbf{A}(f) \cap \mathrm{GL}(\mathcal{U})$ contains a subgroup $H$, such that $W$ is the unique maximal $H$-space in $V$. But $M$ is an $H$-space too and therefore $M=V$. By Remark $2.5 f$ is a quadratic form, a contradiction. 
Lemma 4.12 Let $V$ be a finite-dimensional $\mathbb{F}_{p}$-space, $Q$ a non-degenerate quadratic form on $V$ and $f: V \rightarrow \mathbb{F}_{p}$ be a proper parabolic bent function with respect to the subspace $W$ of $V$.

(a) Let $f=Q_{W, a}^{b}$ be standard parabolic. Then, $\mathbf{E}(f)=\beta(W)$ if $b=1$ and $\mathbf{E}(f)=$ $\beta(\operatorname{rad}(W))$ otherwise.

(b) Let $f=Q_{W, \Pi}$ be twisted parabolic. Then, $\mathbf{E}(f)=\beta(\widetilde{W})$, where $\left.\widetilde{W}=C_{W}(\widetilde{\Pi})\right) \cap$ $\{w \in W \mid Q(w)=Q(w \Pi)\}$.

(c) Let $f$ be special parabolic. Then, $\mathbf{E}(f)=\beta(W)$.

Proof The assertion of the lemma follows from Examples 3.4, 3.6 and 3.8, and Lemmas 2.2 and 4.11, if $W=\operatorname{rad}(W)$ or if $f=Q_{W, a}^{1}$; in particular, assertion (c) holds.

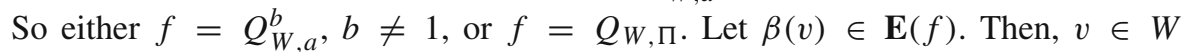
(Lemma 4.11) and there exists a linear functional $\lambda_{v}: V \rightarrow \mathbb{F}_{3}$, such that

$$
f(x+v)=f(x)+x \lambda_{v}+f(v), \quad \text { all } \quad x \in V .
$$

Assume first $f=Q_{W, a}^{b}$. For $x \in W$, we get $Q(x)+Q(v)+(v, x)=Q(x+v)=$ $Q(x)+x \lambda_{v}+f(v)$ or $($ as $f(v)=Q(v))$

$$
(v, x)=x \lambda_{v} .
$$

For $x \notin W$, we get $b(Q(x)+Q(v)+(v, x))+a=b Q(x)+a+x \lambda_{v}+Q(v)$ or

$$
b(v, x)=x \lambda_{v}+(1-b) Q(v)
$$

Replacing $x$ by $-x$ and subtracting, we get $b(v, x)=x \lambda_{v}$ for all $x \in V-W$. Since $V-W$ generates $V$, we have $b(v, x)=x \lambda_{v}$ for $x \in W$ too. As $b \neq 1$, this forces $v \in \operatorname{rad}(W)$ and assertion (a) holds.

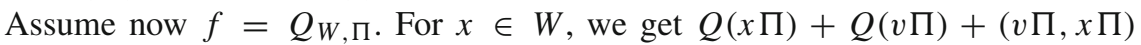
$=Q((x+v) \Pi)=Q(x \Pi)+x \lambda_{v}+f(v)$ or $($ as $f(v)=Q(v \Pi))$

$$
\left(v \Pi \Pi^{*}, x\right)=x \lambda_{v} .
$$

For $x \notin W$, we have $Q(x)+Q(v)+(v, x)=Q(x+v)=Q(x)+x \lambda_{v}+f(v)$ or

$$
(v, x)=x \lambda_{v}+Q(v \Pi)-Q(v) .
$$

Replacing $x$ by $-x$ and subtracting, we get $(v, x)=x \lambda_{v}$ for all $x \in V-W$ and therefore even for all $x$. Then, $Q(v \Pi)=Q(v)$ holds too. This forces $v \in \widetilde{W}$. Conversely, it is easy to see that elements in $\widetilde{W}$ define elements in $\mathbf{E}(f)$.

\subsection{Proof of Theorem 4.1 and its Corollaries}

Proof (Of Theorem 4.1) This theorem follows from Lemmas 4.7, 4.8, 4.9, 4.10 and 4.12. 
Proof (Of Corollary 4.2) By Corollary 2.4, $f_{1}$ and $f_{2}$ are linear equivalent. Assertion (a) follows.

By Remark 3.12 and Theorem 4.1 $\mathbf{A}\left(f_{i}\right) \leq \mathrm{GL}(\mathcal{U})$, these groups leave invariant the decomposition $\mathcal{U}=V \oplus \mathcal{U}_{0}$ (with the usual conventions) and $W^{i}$ is the unique maximal $\mathbf{A}\left(f_{i}\right)$-space in $V(i=1,2)$. Moreover, if $\operatorname{dim} V / W^{i}=m_{i}$, then $\mathbf{A}\left(f_{i}\right)$ induces on $V / W^{i}$ the group $\operatorname{GL}\left(m_{i}, p\right)$, if $f_{i}$ is standard or twisted, whereas a subgroup of $\Gamma \mathrm{L}\left(2,3^{m}\right), m \geq 2$. $\operatorname{dim} V=4 m$ is induced, if $f_{i}$ is special parabolic. Assertion (d) follows.

So let $f_{1}$ and $f_{2}$ be standard or twisted parabolic. By Lemma 4.4 and Remark 2.11, we have $\operatorname{dim} \operatorname{rad}\left(W^{1}\right)=\operatorname{dim} \operatorname{rad}\left(W^{2}\right)$ and $\operatorname{dim} W^{1}=\operatorname{dim} W^{2}$. If $\operatorname{rad}\left(W^{i}\right) \subset W^{i}$, then $f_{i}$ is a quadratic form on $W^{i} / \operatorname{rad}\left(W^{i}\right)$ which has the same type as $Q_{i}$. The linear equivalence of $f_{1}$ and $f_{2}$ shows that the spaces $W^{1} / \operatorname{rad}\left(W^{1}\right)$ and $W^{2} / \operatorname{rad}\left(W^{2}\right)$ are isometric. Hence, $Q_{1}$ and $Q_{2}$ have the same type and Assertions (b) and (c) follow.

Proof (Of Corollary 4.3) (a), (b) and (c). Let $W_{1}=\operatorname{rad}(W)$ be the radical of $W$, $W=W_{1} \oplus W_{0}$ and let $W_{2} \subseteq W_{0}^{\perp}$ be a totally singular complement of $W$ in $V$.

We first show that the given conditions are sufficient for equivalence. We start by considering $Q_{W, a}^{b}$ and $Q_{W, a^{\prime}}^{b}$. Let $\Phi_{0}$ be a similarity of $W_{0}$ with multiplier $r$. Define $\left(w_{0}+w_{1}+w_{2}\right) \Phi=w_{0} \Phi_{0}+w_{1}+r w_{2}$ for $w \in W$ and $w_{i} \in W_{i}$. By Remark 2.7(c), $\Phi$ is a similarity with multiplier $r$ and one observes $Q_{W, a}^{b}(x \Phi)=r Q_{W, a r^{-1}}^{b}(x)$. So by Remark 2.7(b), $Q_{W, a}^{b}$ is linear equivalent to $Q_{W, a^{\prime}}^{b}$ if $a F^{*}=a^{\prime} F^{*}$ in case $\operatorname{dim} V$ is even and if $a\left(F^{*}\right)^{2}=a^{\prime}\left(F^{*}\right)^{2}$ in case $\operatorname{dim} V$ is odd.

Consider next twisted parabolics $Q_{W, \Pi}$ and $Q_{W, \Pi^{\prime}}$, and assume that there exist similarities $\Phi_{1}, \Phi_{2} \in \mathrm{GO}\left(W_{0}, Q\right)$ with multiplier $r$ such that $\Phi_{1} \Pi=\Pi^{\prime} \Phi_{2}$. Define $\Phi \in \operatorname{GL}(V)$ by $\Phi=\operatorname{diag}\left(\Phi_{1}, 1_{W_{1}}, r 1_{W_{2}}\right)$. One observes $r Q_{W, \Pi^{\prime}}(x)=Q_{W, \Pi}(x \Phi)$, i.e., both functions are linear equivalent.

Finally, consider the standard parabolic $Q_{W, 0}^{b}$ and the twisted parabolic $Q_{W, \Pi}$ and assume that $\Pi \in \mathrm{GO}\left(W_{0}, Q_{W_{0}}\right)$ has multiplier $b^{-1}$. Then, $b Q_{W, \Pi}(x)=Q_{W}^{b}(x)$ for all $x \in V$. Again both functions are linear equivalent.

We turn now to the necessity condition for equivalence of two parabolic bent functions (standard or twisted) $f$ and $f^{\prime}$. By Corollary 4.2, they are linear equivalent. Moreover, by Corollary 2.4 and its proof, there exists $\Phi \in \mathrm{GL}(\mathcal{U})$ such that $V \Phi=V$, $\mathcal{U}_{0} \Phi=\mathcal{U}_{0}\left(\mathcal{U}\right.$ and $\mathcal{U}_{0}$ as usual $)$ and $\Gamma\left(f^{\prime}\right)=\Gamma(f) \Phi$. This implies $\Phi^{-1} \mathbf{A}(f) \Phi=$ $\mathbf{A}\left(f^{\prime}\right)$, and this group is isomorphic to a subgroup of $\operatorname{GO}(V, Q)_{W}$. By Theorem 4.1, $\Phi$ normalizes a group $\mathcal{Q} H$, where $\mathcal{Q}$ acts trivially on $\operatorname{rad}(W), W / \operatorname{rad}(W)$ and $V / W$ and $H$ induces the identity on $W / W_{1}\left(\simeq W_{0}\right)$ and the restriction $H_{V}$ lies in the stabilizer of $W$ in $\mathrm{O}(V, Q)$ and by Theorem $4.1 H_{W_{1}} \simeq H_{V / W} \simeq \operatorname{GL}\left(W_{1}\right)$. Let $S \leq H$ be a cyclic group of order $\left|W_{1}\right|-1$. It induces a Singer group on $W_{1}$. Our usual Frattini argument shows that we can assume that $\Phi$ centralizes $S$. So $\Phi$ fixes $W_{1}$, $W_{0}=C_{V}(S)$ and the unique $S$-complement $W_{2}$ of $W$ in $V$ ( $W_{1}$ and $W_{2}$ are not isomorphic as $\mathbb{F}_{p} S$-modules!). Then we adjust $\Phi$ by an element in $S$ so that we have $\Phi_{W_{1}}=1_{W_{1}}$. Then, $\Phi_{W_{2}}=r 1_{W_{2}}, r \in \mathbb{F}_{p}^{*}$, as $\Phi$ induces the trivial automorphism of $H_{V / W}$. Let $\Phi_{\mathcal{U}_{0}}=d \in \mathbb{F}_{p}^{*}$, i.e.,

$$
f\left(w_{0} \Phi_{W_{0}}+w_{1}+r w_{2}\right)=d f^{\prime}\left(w_{1}+w_{2}+w_{2}\right), \quad w_{i} \in W_{i}
$$


Assume first $f=Q_{W, a}^{b}, f^{\prime}=Q_{W, a^{\prime}}^{b^{\prime}}$. If $w_{2}=0$, then

$$
d Q\left(w_{0}\right)=Q\left(w_{0} \Phi_{W_{0}}\right)
$$

and if $w_{2} \neq 0$, then

$$
d\left(b^{\prime}\left(\left(w_{1}, w_{2}\right)+Q\left(w_{0}\right)\right)+a^{\prime}\right)=b\left(\left(w_{1}, r w_{2}\right)+Q\left(w_{0} \Phi_{W_{0}}\right)\right)+a
$$

So if $W \neq W_{1}=\operatorname{rad}(W)$, then $\Phi_{W_{0}}$ is a similarity with multiplier $d$. In this case, the second equation implies $r=d, b=b^{\prime}$ and $a^{\prime} d=a$. Assertion (a) follows in this case. But if $W=W_{1}$, then $a \neq 0 \neq a^{\prime}$ and by convention $b=b^{\prime}=1$ and we are done again, as $\operatorname{dim} V$ is even.

Assume now that $f=Q_{W, \Pi}$ and $f^{\prime}=Q_{W, \Pi^{\prime}}$. Write $x \in V$ as $x=$ $w_{0}+w_{1}+w_{2}$ with $w_{i} \in W_{i}$. If $w_{2}=0$, then $d Q\left(w_{0} \Pi^{\prime}\right)=d f^{\prime}(x)=f(x \Phi)=$ $Q\left(w_{0} \Pi^{\prime}\left(\left(\Pi^{\prime}\right)^{-1} \Phi_{W_{0}} \Pi\right)\right)$, i.e., $\left(\Pi^{\prime}\right)^{-1} \Phi_{W_{0}} \Pi$ lies in $\operatorname{GO}\left(W_{0}, Q\right)$ and has multiplier $d$.

If $w_{2} \neq 0$, then we get $Q\left(w_{0} \Phi_{W_{0}}\right)+\left(w_{1}, r w_{2}\right)=d\left(Q\left(w_{0}\right)+\left(w_{1}, w_{2}\right)\right)$. So $r=d$ and $\Phi_{W_{0}}$ lies in $\operatorname{GO}\left(W_{0}, Q\right)$ and has multiplier $r$. With $\Phi_{1}=\Phi_{W_{0}}$ and $\Phi_{2}=\left(\Pi^{\prime}\right)^{-1} \Phi_{W_{0}} \Pi$ assertion (b) follows.

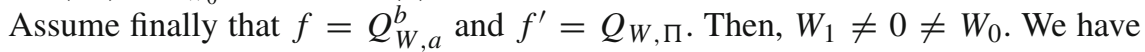
$Q\left(w_{0} \Phi_{W_{0}}\right)=f\left(w_{0} \Phi_{W_{0}}+w_{1}\right)=d f^{\prime}\left(w_{0}+w_{1}\right)=d Q\left(w_{0} \Pi\right)$ and for $w_{2} \neq 0$ we get

$$
b\left(Q\left(w_{0} \Phi_{W_{0}}\right)+\left(w_{1}, r w_{2}\right)\right)+a=d\left(Q\left(w_{0}\right)+\left(w_{1}, w_{2}\right)\right) .
$$

Replacing $w_{1}$ by $-w_{1}$ and subtracting the equations, we obtain $\left(w_{1}, r w_{2}\right)$ $=d\left(w_{1}, w_{2}\right)$ and $b Q\left(w_{0} \Phi_{W_{0}}\right)+a=d Q\left(w_{0}\right)$ for all $w_{0} \in W_{0}$. So $a=0$ and using the first equation, we have $d Q\left(w_{0} \Pi\right)=Q\left(w_{0} \Phi_{W_{0}}\right)=b^{-1} d Q\left(w_{0}\right)$. Hence, $\Pi \in \mathrm{GO}\left(W_{0}, Q_{W_{0}}\right)$ has multiplier $b^{-1}$.

(d) First, we would like to show that the conditions for $\sigma$ and $\sigma^{\prime}$ force the equivalence of $f=Q^{\sigma}$ and $f^{\prime}=Q^{\sigma^{\prime}}$. Let $k_{i} \in F$ with $\operatorname{Tr}\left(k_{i}\right)=i, i=0,1,2$. Then, the $w_{i}=\left(k_{i}, 0,0,1\right)$ 's are representatives of the orbits $B_{i}$ of $\mathcal{Q} H$ in $V-W$. Note that $Q^{\sigma}\left(w_{i}\right)=i^{\sigma}$. Clearly, $-Q^{\sigma}=Q^{(2,3) \sigma}$, i.e., $Q^{\sigma}$ and $Q^{(2,3) \sigma}$ are linear equivalent. We are left with the case that $n$ is even and $0^{\sigma} \neq 0 \neq 0^{\sigma^{\prime}}$. As $\operatorname{Sym}(3)=\langle(2,3)\rangle \cup$ $\langle(2,3)\rangle \sigma\langle(2,3)\rangle$, there exist $\pi_{1}, \pi_{2} \in\langle(2,3)\rangle$ with $\sigma \pi_{1}=\pi_{2} \sigma^{\prime}$. Also there exist $\epsilon_{j} \in \mathbb{F}_{3}^{*}$ with $i^{\pi_{j}}=\epsilon_{j} i$ for $i \in \mathbb{F}_{3}, j=1,2$. Let $\mathcal{U}$ and $\mathcal{U}_{0}$ be defined as usual. Define $\Phi \in \operatorname{GL}(\mathcal{U})$ by $\Phi_{V}=1_{V}$ and $\Phi_{\mathcal{U}_{0}}=\epsilon_{1}$ if $\epsilon_{2}=1$ and by $\Phi_{V}=z(v)_{V}$ and $\Phi_{\mathcal{U}_{0}}=\epsilon_{1}$ if $\epsilon_{2}=-1$. Then, $\Phi_{V} \in N_{\mathrm{GL}(V)}\left((\mathcal{Q} H)_{V}\right)$ (cf. Lemma 4.9) and $\Phi$ maps $\left(w_{i}, i^{\sigma}\right)=\left(k_{i}, 0,0,1, i^{\sigma}\right) \in \Gamma(f)$ onto $\left(\epsilon_{2} k_{i}, 0,0,1, \epsilon_{1} i^{\sigma}\right)=\left(w_{\epsilon_{2} i}, \epsilon_{1} i^{\sigma}\right)$. As $\left(w_{\epsilon_{2} i}, \epsilon_{1} i^{\sigma}\right)=\left(w_{i_{2}}, i^{\sigma \pi_{1}}\right)=\left(w_{i_{2}},\left(i^{\pi_{2}}\right)^{\sigma^{\prime}}\right)$,we get $\left(w_{i}, i^{\sigma}\right) \Phi \in \Gamma\left(f^{\prime}\right)$. Since $\Phi_{V}$ normalizes $(\mathcal{Q} H)_{V}$, we see that $\Phi$ maps $\Gamma\left(Q^{\sigma}\right)$ onto $\Gamma\left(Q^{\sigma^{\prime}}\right)$.

Assume now that $f=Q^{\sigma}$ and $f^{\prime}=Q^{\sigma^{\prime}}$ are EA equivalent. By Corollary 4.2, they are linear equivalent and there exists $\Phi \in \mathrm{GL}(\mathcal{U})$ that fixes the spaces $W, V$ and $\mathcal{U}_{0}$ and maps $\Gamma(f)$ onto $\Gamma\left(f^{\prime}\right)$. Also because of this corollary and the first part of this verification, we only have to consider the case that $n$ is odd. Then, $\mathbf{A}(f)=\mathbf{A}\left(f^{\prime}\right)=$ $\Phi^{-1} \mathbf{A}(f) \Phi$ by Theorem 4.1 and hence $\Phi_{V} \in N_{\mathrm{GL}(V)}\left((\mathcal{Q}) H_{V}\right)$. By Claim (*) in the 
proof of Lemma 4.9 and Theorem 4.1, we may assume $\Phi_{V}=1_{V}$ or $=z(v)_{V}(n$ is even). But as $n$ is odd, we see $\Phi=\operatorname{diag}\left(1_{V}, \pm 1\right)$ and assertion (c) follows.

Remark 4.13 Let $V$ and $W$ be as in Corollary 4.3. The equivalence classes of standard parabolic bent functions with respect to $W$ are represented by $Q_{W, 0}^{1}$ and $Q_{W, 1}^{b}, b \in \mathbb{F}_{p}^{*}$ if $\operatorname{dim} V$ is even and by $Q_{W, 0}^{1}, Q_{W, 1}^{b}, Q_{W, v}^{b}$, for $b \in \mathbb{F}_{p}^{*}$ and some $v \in \mathbb{F}_{p}^{*}-\left(\mathbb{F}_{p}^{*}\right)^{2}$ if $\operatorname{dim} V$ is odd.

Let $V=V(4 n, 3)$. The equivalence classes of special parabolic bent functions are represented by $Q^{1}$ and $Q^{(0,1)}$ if $n$ is even and by $Q^{1}, Q^{(0,1)}$ and $Q^{(0,2)}$ if $n$ is odd.

\section{Automorphism groups and equivalence of Maiorana functions}

In this section, we will clarify the automorphism and equivalence problem for the Maiorana McFarland functions from Example 3.13. We shall prove:

Theorem 5.1 Let $p>2$ be a prime, $V=F \times F, F=\mathbb{F}_{p^{n}}, 0<\ell<p^{n}-1$ be coprime to $p^{n}-1$ and let $\mu_{\ell}: V \rightarrow \mathbb{F}_{p}$ be the $p$-ary bent function defined by $\mu_{\ell}(x, y)=\operatorname{Tr}\left(x y^{\ell}\right)$. Assume that $\ell$ is not congruent to a p-power modulo $p^{n}-1$. Then, $\mathbf{E A}\left(\mu_{\ell}\right)=\mathbf{A}\left(\mu_{\ell}\right) \leq \operatorname{GL}(\mathcal{U}), \mathcal{U}=\mathcal{U}\left(\mu_{\ell}\right)$. Moreover, $\mathbf{A}\left(\mu_{\ell}\right)=\mathcal{Q Z \mathcal { P }}\langle\Theta\rangle$ and $\mathcal{Q}=O_{p}\left(\mathbf{A}\left(\mu_{\ell}\right)\right)$ is described in Sect. 5.1.

Corollary 5.2 Let $p>2$ be a prime, $V=F \times F, F=\mathbb{F} p^{n}$, and let $0<\ell, m<p^{n}-1$ be coprime to $p^{n}-1$. Define $p$-ary bent functions $\mu_{\ell}, \mu_{m}: V \rightarrow \mathbb{F}_{p}$ by $\mu_{s}(x, y)=$ $\operatorname{Tr}\left(x y^{s}\right)$ for $s=\ell$ or $=m$. The following hold:

(a) Let $\ell \equiv m p^{a}\left(\bmod p^{n}-1\right)$ for some number $a$. Then, $\mu_{\ell}$ and $\mu_{m}$ are linear equivalent.

(b) Let $\mu_{\ell}$ and $\mu_{m}$ be EA equivalent. Then, $\ell \equiv m p^{a}\left(\bmod p^{n}-1\right)$ for some number $a$.

We start with simple observations.

Lemma 5.3 Let $p$ be an odd prime and $\ell \nsim 1$. Then, $\mathbf{E}\left(\mu_{\ell}\right)=1$ and $\mathbf{E A}\left(\mu_{\ell}\right)=$ $\mathbf{A}\left(\mu_{\ell}\right) \leq \operatorname{GL}(\mathcal{U}), \mathcal{U}=\mathcal{U}\left(\mu_{\ell}\right)$.

Proof Assume $\mathbf{E}\left(\mu_{\ell}\right) \neq 1$ and pick $1 \neq \beta\left(x_{0}, y_{0}\right) \in \mathbf{E}\left(\mu_{\ell}\right)$. We know

$$
(x, y, z) \beta\left(x_{0}, y_{0}\right)=\left(x+x_{0}, y+y_{0}, z+(x, y) \lambda+\mu_{\ell}(x, y)\right)
$$

with $\lambda=\lambda_{x_{0}, y_{0}}$ a linear functional, which we write as $(x, y) \lambda=\operatorname{Tr}(a x+b y)$ (cf. Remark 2.5). So

$$
\mu_{\ell}\left(x+x_{0}, y+y_{0}\right)=\mu_{\ell}(x, y)+\operatorname{Tr}(a x+b y)+\mu_{\ell}\left(x_{0}, y_{0}\right)
$$

for all $x, y \in F$. We have $\pi_{-1} \beta\left(x_{0}, y_{0}\right) \pi_{-1}=\beta\left(-x_{0}, y_{0}\right)\left(\pi_{-1}\right.$ is defined in Example 3.13). Moreover, $(x, y) \lambda_{-x_{0}, y_{0}}=\operatorname{Tr}(a x-b y)$. Replace $\beta\left(x_{0}, y_{0}\right)$ by $\beta\left(x_{0}, y_{0}\right) \pi_{-1} \beta\left(x_{0}, y_{0}\right) \pi_{-1}$ or by $\beta\left(x_{0}, y_{0}\right)^{-1} \pi_{-1} \beta\left(x_{0}, y_{0}\right) \pi_{-1}$, so that we may either 
assume (1) $x_{0}=b=0 \neq y_{0}, a$ or (2) $y_{0}=a=0 \neq x_{0}, b$. In both cases, $\mu_{\ell}\left(x_{0}, y_{0}\right)=0$.

CASE (1) For all $x, y \in F$ we have $\operatorname{Tr}\left(x\left(y+y_{0}\right)^{\ell}\right)=\operatorname{Tr}\left(x y^{\ell}\right)+\operatorname{Tr}(a x)$. Set $y=-y_{0}$ and we see $a=y_{0}^{\ell}$. The equation becomes

$$
\sum_{i=1}^{\ell-1}\left(\begin{array}{l}
\ell \\
i
\end{array}\right) \operatorname{Tr}\left(x y^{i} y_{0}^{\ell-i}\right)=\operatorname{Tr}\left(x \sum_{i=1}^{\ell-1}\left(\begin{array}{l}
\ell \\
i
\end{array}\right) y^{i} y_{0}^{\ell-i}\right)=0
$$

This shows $\sum_{i=1}^{\ell-1}\left(\begin{array}{c}\ell \\ i\end{array}\right) y^{i} y_{0}^{\ell-i}=0$ and that $\left(\begin{array}{l}\ell \\ i\end{array}\right)=0,1 \leq i<\ell$, i.e., $x \mapsto x^{\ell}$ is additive, so that $\ell \sim 1$, a contradiction.

CASE (2) For all $x, y \in F$, we have $\operatorname{Tr}\left(\left(x+x_{0}\right) y^{\ell}\right)=\operatorname{Tr}\left(x y^{\ell}\right)+\operatorname{Tr}(b y)$, i.e., $\operatorname{Tr}\left(x_{0} y^{\ell}\right)=\operatorname{Tr}(b y)$, which forces $x_{0}=b=0$, a contradiction. So $\mathbf{E}\left(\mu_{\ell}\right)=1$. The second assertion follows from Lemma 2.3.

Lemma 5.4 Let $\mu=\mu_{\ell}$ and $(a, b),(x, y) \in F \times F$. Then,

$$
D_{(a, b)} \mu(x, y)=\operatorname{Tr}\left(x \sum_{i=0}^{\ell-1}\left(\begin{array}{l}
\ell \\
i
\end{array}\right) b^{\ell-i} y^{i}+a \sum_{i=0}^{\ell}\left(\begin{array}{l}
\ell \\
i
\end{array}\right) b^{\ell-i} y^{i}\right)
$$

and

$D_{(a, b)}^{2} \mu(x, y)=\operatorname{Tr}\left(x \sum_{i=0}^{\ell-2}\left(\begin{array}{l}\ell \\ i\end{array}\right)\left(2^{\ell-i}-2\right) b^{\ell-i} y^{i}+a \sum_{i=0}^{\ell-1}\left(\begin{array}{l}\ell \\ i\end{array}\right)\left(2^{\ell-i+1}-2\right) b^{\ell-i} y^{i}\right)$

If $\ell$ is not a $p$-power, then $D_{(a, b)}^{2} \mu=0$ iff $b=0$.

Proof As the trace is additive, we have $D_{(a, b)} \mu(x, y)=\operatorname{Tr}\left((x+a)(y+b)^{\ell}-x y^{\ell}\right)$ and the first assertion follows. By Lemma 2.10:

$$
\begin{aligned}
D_{(a, b)}^{2} \mu(x, y)= & \operatorname{Tr}\left(x \sum_{i=0}^{\ell-1}\left(\begin{array}{l}
\ell \\
i
\end{array}\right)(2 b)^{\ell-i} y^{i}+(2 a) \sum_{i=0}^{\ell}\left(\begin{array}{l}
\ell \\
i
\end{array}\right)(2 b)^{\ell-i} y^{i}\right) \\
& -2 \operatorname{Tr}\left(x \sum_{i=0}^{\ell-1}\left(\begin{array}{l}
\ell \\
i
\end{array}\right) b^{\ell-i} y^{i}+a \sum_{i=0}^{\ell}\left(\begin{array}{l}
\ell \\
i
\end{array}\right) b^{\ell-i} y^{i}\right) \\
= & \operatorname{Tr}\left(x \sum_{i=0}^{\ell-2}\left(\begin{array}{l}
\ell \\
i
\end{array}\right)\left(2^{\ell-i}-2\right) b^{\ell-i} y^{i}+a \sum_{i=0}^{\ell-1}\left(\begin{array}{l}
\ell \\
i
\end{array}\right)\left(2^{\ell-i+1}-2\right) b^{\ell-i} y^{i}\right)
\end{aligned}
$$

Assume now that $\ell$ is not a $p$-power. Clearly, $D_{(a, b)}^{2} \mu(x, y)=0$ if $b=0$.

So assume $b \neq 0$. We claim that $A(Y)=\sum_{i=0}^{\ell-2}\left(\begin{array}{l}\ell \\ i\end{array}\right)\left(2^{\ell-i}-2\right) b^{\ell-i} Y^{i} \in F[Y]$ is not the trivial polynomial.

Let $\ell=\ell_{k} p^{k}+\ell_{k-1} p^{k-1}+\cdots+\ell_{0}$ be the $p$-adic expansion of $\ell$. As $\ell$ is not a $p$ power and coprime to $p^{n}-1$ (in particular, $\ell$ is odd), we have that $\ell_{k}+\ell_{k-1}+\cdots+\ell_{0} \geq$ 
3. By replacing $\ell$ by $p^{c} \ell\left(\bmod p^{n}-1\right)$ for a suitably chosen $c$ if necessary, we may assume $\ell_{0}=\max \left\{\ell_{i} \mid 0 \leq i \leq k\right\}$. Then, $\ell_{0}>0$ and according to the theorem of Lucas $\left(\begin{array}{c}\ell \\ i\end{array}\right) \neq 0$ for $0 \leq i \leq \ell_{0}$. Two consecutive $\left(2^{\ell-i}-2\right)$ 's cannot both be congruent 0 modulo $p$. So for at least one $i \in\left\{0, \ldots, \ell_{0}\right\}$, we have $\left(\begin{array}{c}\ell \\ i\end{array}\right)\left(2^{\ell-i}-2\right) b^{\ell-i} \neq \equiv$ $(\bmod p)$. This proves the claim.

Choose, $y \in F$, such that $A(y) \neq 0$. Then, $D_{(a, b)}^{2} \mu(x, y)$ is the trace of $x A(y)+a B(y)$ with $B(y)=\sum_{i=0}^{\ell-1}\left(\begin{array}{l}\ell \\ i\end{array}\right)\left(2^{\ell-i+1}-2\right) b^{\ell-i} y^{i}$. Choose $x \in F$, such that $\operatorname{Tr}(x A(y)+a B(y)) \neq 0$, i.e., $D_{(a, b)}^{2} \mu(x, y) \neq 0$ and $D_{(a, b)}^{2} \mu$ is not the trivial function.

\subsection{The $p$-radical of $A\left(\mu_{\ell}\right)$}

Lemma 5.5 Let $n>1$ be an integer, $p$ a prime and $\ell \in\left[1, p^{n}-2\right] \cap \mathbb{Z}$ be invertible modulo $p^{n}-1$.

(a) Assume $1+\ell \sim 1+p^{a} \ell \sim 1+p^{x} \ell$ with $a, x \in[0, n-1] \cap \mathbb{Z}, a>1$. Then, $x=0$ or $x=a$.

(b) Let $(n, p) \neq(6,2)$. Then, there exists at most one $a \in[0, n-1] \cap \mathbb{Z}$, such that there exists a proper divisor $d$ of $n$, such that $\left(\ell+p^{a}\right) p^{d} \equiv \ell+p^{a}\left(\bmod p^{n}-1\right)$.

Proof (a) Suppose $1+\ell \equiv p^{b}\left(1+p^{a} \ell\right) \equiv p^{y}\left(1+p^{x} \ell\right)\left(\bmod p^{n}-1\right)$. Then, $a>0$ forces $b>0$ too and $\ell\left(p^{a+b}-1\right) \equiv 1-p^{b}\left(\bmod p^{n}-1\right)$ and $\ell\left(p^{x+y}-1\right) \equiv 1-p^{y}$ $\left(\bmod p^{n}-1\right)$. Hence,

$$
\begin{aligned}
\left(1-p^{y}\right)\left(p^{a+b}-1\right) & \equiv \ell\left(p^{a+b}-1\right)\left(p^{x+y}-1\right) \\
& \equiv\left(1-p^{b}\right)\left(p^{x+y}-1\right) \quad\left(\bmod p^{n}-1\right),
\end{aligned}
$$

which implies

$$
p^{b+x+y}+p^{a+b}+p^{y} \equiv p^{a+b+y}+p^{x+y}+p^{b} \quad\left(\bmod p^{n}-1\right) .
$$

Substitute $a+b$ by $\bar{a}$ and $x+y$ by $\bar{x}$. By [8, (2.3)] either $\bar{a}=\bar{x}$ and $b=y$ or $\bar{x}=y$ and the assertion follows..

(b) Suppose $\left(\ell+p^{a}\right) p^{d} \equiv \ell+p^{a}$ and $\left(\ell+p^{b}\right) p^{e} \equiv \ell+p^{b}\left(\bmod p^{n}-1\right)$ for proper divisors $d$ and $e$ of $n$. Then, $\left(\ell+p^{a}\right)\left(p^{d}-1\right) \equiv 0\left(\bmod p^{n}-1\right)$ and $\left(\ell+p^{b}\right)\left(p^{e}-1\right) \equiv 0\left(\bmod p^{n}-1\right)$. We use Zsigmondy's theorem [27]. Assume that $p^{n}-1$ has a $p$-primitive prime divisor $r$. Then, $r$ divides $\ell+p^{a}$ and $\ell+p^{b}$ and thus $p^{a}-p^{b}$ forcing $a=b$, i.e., the assertion.

We are left with the case $n=2$ and $p+1=2^{c}$. Then, $e=d=1$ and so $\left(p^{a}-p^{b}\right)(p-1) \equiv 0\left(\bmod p^{2}-1\right)$, forcing $a=b$.

Remark 5.6 Let $\mathcal{U}=F \times F \times \mathbb{F}_{p}, F=\mathbb{F}_{p^{n}}$. For $0 \leq a<n$ and $\beta \in F^{*}$, define $t_{a}(\beta)$ in $\operatorname{GL}(\mathcal{U})$ by

$$
(x, y, z) t_{a}(\beta)=\left(x+y T_{a}(\beta), y, z\right)=\left(x+\beta y^{p^{a}}, y, z\right) .
$$


We set $\mathcal{T}_{a}=\left\{t_{a}(\beta) \mid \beta \in F\right\}$.

Suppose $0 \leq a, b<n, a \neq b$ and that there exists a $0 \leq c<n$, such that $\ell+p^{a} \equiv p^{c} \ell+p^{b+c}\left(\bmod p^{n}-1\right)$. Then, $c$ is uniquely determined: Assume $\ell+p^{a} \equiv p^{d} \ell+p^{b+d}\left(\bmod p^{n}-1\right)$. This leads to $\ell\left(p^{c}-1\right) \equiv p^{a}-p^{b+c}\left(\bmod p^{n}-1\right)$ and $\ell\left(p^{d}-1\right) \equiv p^{a}-p^{b+d}\left(\bmod p^{n}-1\right)$. So we get

$\left(p^{c}-1\right)\left(p^{a}-p^{b+d}\right) \equiv \ell\left(p^{c}-1\right)\left(p^{d}-1\right) \equiv\left(p^{d}-1\right)\left(p^{a}-p^{b+c}\right) \quad\left(\bmod p^{n}-1\right)$

or $p^{a+c}+p^{b+d} \equiv p^{a+d}+p^{b+c}\left(\bmod p^{n}-1\right)$, which forces $d=c(\operatorname{as} a \neq b)$.

We claim that $\sigma_{a, b}(\beta)=t_{a}(\beta) t_{b}\left(-\beta^{p^{-c}}\right)$ induces an element of $\mathbf{A}\left(\mu_{\ell}\right)$. We observe

$$
\operatorname{Tr}\left(y\left(T_{a}(\beta)+T_{b}\left(-\beta^{p^{-c}}\right)\right) \cdot y^{\ell}\right)=\operatorname{Tr}\left(\beta y^{p^{a}+\ell}-\beta^{p^{-c}} y^{p^{b}+\ell}\right)=0,
$$

which implies $\mu_{\ell}\left((x, y) \sigma_{a, b, c}(\beta)\right)=\mu_{\ell}(x, y)$. We set

$$
\mathcal{Q}_{a, b}=\left\{\sigma_{a, b}(\beta) \mid \beta \in F\right\} .
$$

One computes (or use Lemma 2.14) $z_{u}^{-1} t_{a}(\beta) z_{u}=t_{a}\left(u^{\bar{\ell}} p^{a}+1 \beta\right)$. Thus, $Z=\left(F^{*}, \cdot\right)$ induces on $\mathcal{T}_{a}$ the representation $D^{\bar{\ell} p^{a}+1}$ (we identify $\beta \in F$ with $t_{a}(\beta)$ ). If $\ell+p^{a} \equiv$ $p^{c} \ell+p^{b+c}\left(\bmod p^{n}-1\right)$, then $1+p^{a} \bar{\ell} \equiv p^{c}+p^{b+c} \bar{\ell}\left(\bmod p^{m}-1\right)$. So $Z$ induces the representation $D^{\bar{\ell} p^{a}+1}$ on $\mathcal{Q}_{a, b}$.

It can happen that the intersection of $\mathbf{A}\left(\mu_{\ell}\right)$ with a group $\mathcal{T}_{a}$ is non-trivial. This will be considered in the next lemma.

Lemma 5.7 Let $0 \leq a<n$ and assume that there exists a proper divisor $d$ of $n$, such that $\left(\ell+p^{a}\right) p^{d} \equiv \ell+p^{a}\left(\bmod p^{n}-1\right)$. If $d$ is chosen minimal with this property, then

$$
\mathbf{A}\left(\mu_{\ell}\right) \cap \mathcal{T}_{a}=\left\{t_{a}(\beta) \mid \beta \in \operatorname{ker} \operatorname{Tr}_{n: d}\right\}
$$

In particular, $\left|\mathbf{A}\left(\mu_{\ell}\right) \cap \mathcal{T}_{a}\right|=p^{n-d}$.

Proof $t_{a}(\beta) \in \mathbf{A}\left(\mu_{\ell}\right) \cap \mathcal{T}_{a}$ is equivalent to

$$
\operatorname{Tr}\left(\beta y^{p^{a}+\ell}\right)=0
$$

for all $y \in F$. Hence, $\beta D^{p^{a}+\ell}\left(F^{*}\right) \subseteq \operatorname{ker} \operatorname{Tr} \subset F$. By Lemma 2.13, we conclude that $p^{a}+\ell$ lies in a short cyclotomic coset of $n$ modulo $p$. So there exists a proper divisor $d$ of $n$, such that $\left(\ell+p^{a}\right) p^{d} \equiv \ell+p^{a}\left(\bmod p^{n}-1\right)$, i.e., $D^{p^{a}+\ell}\left(F^{*}\right) \subseteq \mathbb{F}_{p^{d}}$. Moreover, $\operatorname{dim}\left\langle D^{p^{a}+\ell}\left(F^{*}\right)\right\rangle=d$, if $d$ is chosen minimal with this property: By this assumption, $D^{p^{a}+\ell}\left(F^{*}\right)$ and so $E=\left\langle D^{p^{a}+\ell}\left(F^{*}\right)\right\rangle$ lies in no proper subfield of $\mathbb{F}_{p^{d}}$. But $E$ is a ring, i.e., a subfield and $E=\mathbb{F}_{p^{d}}$ follows.

Let $\beta \in \operatorname{ker} \operatorname{Tr}_{n: d} \subseteq \operatorname{ker} \operatorname{Tr}$. Then, $\beta y^{p^{a}+\ell} \in \operatorname{ker} \operatorname{Tr}_{n: d}$, as $\operatorname{ker} \operatorname{Tr}_{n: d}$ is an $\mathbb{F}_{p^{d} \text {-space. }}$ Hence, $\operatorname{Tr}\left(\beta y^{p^{a}+\ell}\right)=0$ for all $y \in F$ and $t_{a}(\beta) \in \mathbf{A}\left(\mu_{\ell}\right)$.

On the other hand, $t_{a}(\beta) \in \mathbf{A}\left(\mu_{\ell}\right)$ implies $\beta D^{p^{a}+\ell}\left(F^{*}\right) \subseteq$ ker Tr, i.e., $\beta$ lies in an $\mathbb{F}_{p^{d}}$-subspace of ker Tr and we have already $\beta \in \operatorname{ker} \operatorname{Tr}_{n: d}$ if $d=1$. If $d>1$, then 
$\operatorname{ker} \operatorname{Tr}_{n: d}$ is a maximal $\mathbb{F}_{p^{d}}$-subspace of ker $\operatorname{Tr}$ (note that ker $\operatorname{Tr}$ is not an $\mathbb{F}_{p^{d}}$-space), we obtain again $\beta \in \operatorname{ker} \operatorname{Tr}_{n: d}$.

Let $p>2$ be a prime and set $\mathcal{I}=[0, n-1] \cap \mathbb{Z}$. By Example 3.13, Remark 5.6 and Lemmas 5.5 and 5.7, there exists a partition $\mathcal{I}=\mathcal{P} \cup \mathcal{E} \cup(\mathcal{I}-(\mathcal{P} \cup \mathcal{E}))$, such that there exists a partition $\mathcal{P}=\left\{a_{1}, b_{1}\right\} \cup \cdots \cup\left\{a_{s}, b_{s}\right\}$ into 2-sets, such that $\left|\mathcal{Q}_{a_{i} b_{i}}\right|=p^{n}$ for $1 \leq i \leq s$ and $\mathcal{Q}_{a}=\mathcal{T}_{a} \cap \mathbf{A}\left(\mu_{\ell}\right) \neq 1$ for $a \in \mathcal{E}$. Moreover, both sets $\mathcal{P}$ and $\mathcal{E}$ may be empty and $|\mathcal{E}| \leq 1$ by Lemma $5.5(\mathrm{~b})$. We set

$$
\mathcal{Q}=\prod_{i=1}^{s} \mathcal{Q}_{a_{i}, b_{i}} \cdot \prod_{a \in \mathcal{E}} \mathcal{Q}_{a} .
$$

Lemma 5.8 Let $Q$ be the subgroup of operators $\Phi \in \mathbf{A}\left(\mu_{\ell}\right)$, such that $\Phi$ induces the identity on $X, V / X$ and $\mathcal{U} / V$. Then $Q=\mathcal{Q}$.

Proof Let $\Phi$ be an element of $Q$. Our assumption forces $\Phi \in \mathcal{T}:=\prod_{i=0}^{n-1} \mathcal{T}_{i}$. The group $Z=\left(F^{*}, \cdot\right)$ induces the representation $D^{1}$ on $X, D^{-\bar{\ell}}$ on $Y$, and $D^{1+p^{a} \bar{\ell}}$ on $\mathcal{T}_{a}$ by Remark 5.6. By Lemma 5.5, a representation $D^{1+p^{a} \bar{\ell}}$ occurs either once or twice in $\mathcal{T}$. Define $\mathcal{P}$ and $a_{i}, b_{i}, 1 \leq i \leq s$ as above. Then, $\mathcal{T}=\prod_{i=1}^{s}\left(\mathcal{T}_{a_{i}} \mathcal{T}_{b_{i}}\right) \prod_{a \in \mathcal{I}-\mathcal{P}} \mathcal{T}_{a}$ is the decomposition of $\mathcal{T}$ into homogeneous components with respect to $\mathcal{Z}$. Thus, $Q=\prod_{i=1}^{s}\left(Q \cap \mathcal{T}_{a_{i}} \mathcal{T}_{b_{i}}\right) \prod_{a \in \mathcal{I}-\mathcal{P}}\left(Q \cap \mathcal{T}_{a}\right)$ by Clifford's Theorem. But obviously, $\mathcal{Q}_{a_{i}, b_{i}}=Q \cap \mathcal{T}_{a_{i}} \mathcal{T}_{b_{i}}$ and $\mathcal{Q}_{a}=Q \cap \mathcal{T}_{a}$ showing the assertion.

\subsection{Proof of Theorem 5.1 and Corollary 5.2}

Lemma 5.9 Let $p$ be a prime, $V=V(n, p), n=m a>1$, and $S \leq G=\operatorname{GL}(V) a$ Singer group.

(a) G contains precisely one conjugacy class $\mathcal{C}$ of subgroups isomorphic to $\operatorname{SL}\left(m, p^{a}\right)$, which act transitively on $\mathrm{V}-0$.

(b) There exists precisely one element in $\mathcal{C}$, whose normalizer contains $S$.

Proof (a) follows from the classification of 2-transitive permutation groups [5], Hering's Theorem [20], but also from the fact that $\operatorname{SL}\left(m, p^{a}\right)$ has only one simple module over $\mathbb{F}_{p}$ of size $p^{n}$, such that all non-trivial elements of the module are conjugate (cf. [22]).

(b) Assume $S \leq N_{G}(H) \cap N_{G}\left(H_{1}\right)$ with $H$ and $H_{1} \in \mathcal{C}$. Let $H_{1}=H^{g}$. Then, $S$ and $S^{g}$ are Singer groups in $N_{G}\left(H_{1}\right) \simeq \Gamma \mathrm{L}\left(m, p^{a}\right)$. By Lemma 2.12, there exists $h \in N_{G}\left(H_{1}\right)$ with $S=S^{g h}$. But then $g h \in N_{G}(H)$, as $N_{G}(S) \leq N_{G}(H)$. Hence, $H_{1}=H^{g}=H^{g h}=H$.

Identify $V=V(n, p)$ with $(F,+), F=\mathbb{F}_{p^{n}}$. Then, $S=T_{0}\left(F^{*}\right)$ is a Singer group in $\operatorname{GL}(V)$. Let $\mathcal{H}$ be the set of operators $T((b)), b=\left(b_{0}, \ldots, b_{n-1}\right) \in F^{n}$ with $b_{i}=0$ for $i \not \equiv 0(\bmod a)$. In the obvious way, $\mathcal{H}$ can be identified with the set of $\mathbb{F}_{p^{a}}$-linear endomorphisms of $V$. So the invertible elements of $\mathcal{H}$ form a group isomorphic to $\operatorname{GL}\left(m, p^{a}\right)$ and the unique subgroup $H \simeq \operatorname{SL}\left(m, p^{a}\right)$ has the property $S \leq N_{\mathrm{GL}(V)}(H)$. 
Lemma 5.10 Let $V=V(n, q), n>1$ and $S=\operatorname{SL}(V)$. Then, $\Gamma \mathrm{L}(V)$ is the normalizer of $S$ in the symmetric group $\operatorname{Sym}(V)$.

Proof We exclude the cases $(n, q)=(2,2)$ and $(2,3)$, which can be treated in an ad hoc fashion, for instance by computer calculation with GAP [25]. Set $N=N_{\operatorname{Sym}(V)}(S)$ and $H=\Gamma \mathrm{L}(V)$. Clearly, $H \leq N$. The group $N$ fixes 0 , the only fixed-point of $S$, and permutes the sets $\mathbb{F}_{q} v-\{0\}, 0 \neq v \in V$ : Assume $\alpha \in N$, such that $(F v) \alpha \cap F v \neq$ $\{0\} \neq(F v) \alpha \cap F w$ for $F v \neq F w$. Note that $(F v) \alpha-\{0\}$ is a set of imprimitivity of $S$, since $F v-\{0\}$ is such a set. However, the pointwise stabilizer of $F v$ in $S$ acts transitively on points $\neq F v$, i.e., we find some $\beta \in S$, such that $(F v) \alpha \beta \cap(F v) \alpha \neq\{0\}$ and $(F v) \alpha \beta \neq(F v) \alpha$ as there are more than $q$ points, a contradiction.

Set $\Omega=\left\{\mathbb{F}_{q} v \mid 0 \neq v \in V\right\}$. Let $K$ be the kernel of the action of $N$ on $\Omega$. We use the bar convention for homomorphic images modulo $K$, in particular $\bar{N}=N / K$ and $\bar{S}=S K / K \simeq S /(S \cap K) \simeq \operatorname{PSL}(V)$ is simple. Then, $\bar{N}$ is a subgroup of $\operatorname{Aut}(\bar{S})$, as $(\bar{S}, \Omega)$ is a primitive permutation group. One knows $\operatorname{Aut}(\bar{S})=\bar{H}$ if $n=2$ and $\operatorname{Aut}(\bar{S})=\bar{H}\langle\gamma\rangle$ if $n>2$ (see [6, Thm. 12.5.1]). Here, $\gamma$ is the graph automorphism of $\bar{S}$. But if $\bar{S}_{\omega}, \omega \in \Omega$, is a point stabilizer, then $\bar{S}_{\omega}^{\gamma}$ is the stabilizer of a hyperplane. Since these two groups have orbits of different sizes on $\Omega$, we conclude $\bar{N}=\bar{H}$.

Let $\alpha \in N$. Adjusting $\alpha$ by an element from $H$, we may assume that $\alpha$ induces the trivial automorphism of $\bar{S}$, i.e., $[S, \alpha]=[\alpha, S] \leq Z(S)$. Then, by the ThreeSubgroup Lemma $[1,(8.7)][S, \alpha]=[[S, S], \alpha]=[[S, \alpha], S]=[[\alpha, S], S]=1$, i.e., $\alpha \in C_{\operatorname{Sym}(V)}(S)$. The stabilizer of a point $\mathbb{F}_{q} v$ in $S$ induces $\operatorname{GL}(1, q)$ on this point. So there exists a scalar $0 \neq a_{v} \in \mathbb{F}_{q}^{*}$, such that $(c v) \alpha=a_{v}(c v), c \in \mathbb{F}_{q}$. If $v$ and $w$ are linearly independent, there exists $\Phi \in S$ with $v \Phi \in \mathbb{F}_{q} w$. Hence, $a_{w}(v \Phi)=(v \Phi) \alpha=(v \alpha) \Phi=\left(a_{v} v\right) \Phi$ and $a_{v}=a_{w}$. So $\alpha=a 1_{v}, a \in \mathbb{F}_{q}^{*}$, i.e., $\alpha \in H$.

Proof (Of Theorem 5.1) Set $\mu=\mu_{\ell}$. By Lemma 2.3, $G=\mathbf{A}(\mu) \leq \operatorname{GL}(\mathcal{U}), \mathcal{U}=$ $F \times F \times \mathbb{F}_{p}, F=\mathbb{F}_{p^{n}}$. Set $X=F \times 0 \times 0, Y=0 \times F \times 0$ and $\mathcal{U}_{0}=0 \times 0 \times \mathbb{F}_{p}$, i.e., $V=X \oplus Y$. By Lemma 5.4, $X$ is invariant under $G$. Note that $C_{M}\left(O_{p}(G)\right) \neq 0$ by $[1,(5.15)]$ and $M$ is an irreducible $\mathcal{Z}$-module for $M \in\left\{X, V / X, \mathcal{U}_{0}\right\}$. This shows $C_{M}\left(O_{p}(G)\right)=M$ and therefore $\mathcal{Q}=O_{p}(G)$ by Lemma 5.8.

By Lemma $5.8, G / \mathcal{Q}$ is isomorphic to a subgroup of $\operatorname{GL}(X) \times \operatorname{GL}(V / X)$. Set $\mathcal{T}=\mathcal{Z}_{0} \mathcal{P}$, where $\mathcal{Z}_{0}$ is the subgroup of $\mathcal{Z}$ of order $p-1$. Then, $\mathcal{T} \mathcal{Q} / \mathcal{Q} \simeq Z(\operatorname{GL}(X) \times$ $\mathrm{GL}(V / X))$, i.e., $\mathcal{T} \mathcal{Q} \unlhd G$. A Frattini argument shows $G=N_{G}(\mathcal{T}) \mathcal{Q}$. Set $N=N_{G}(\mathcal{T})$. Then, $V=X \oplus Y$ is an $N$-invariant decomposition (as $X$ and $Y$ are the homogeneous components of $\mathcal{T}$ on $V$ ). Clearly, $\mathcal{Z} \mathcal{P}\langle\Theta\rangle \leq N$ and $\mathcal{Z}\langle\Theta\rangle \leq N_{0}$, where $N_{0}$ is the kernel of the action of $N$ on $\mathcal{U}_{0}$.

We have to show $N_{0}=\mathcal{Z}\langle\Theta\rangle$. The group $N_{0}$ is faithfully represented on $X$ as well as on $Y$, since $\mu(x, y)=\mu\left(x \Phi_{X}, y \Phi_{Y}\right)$ for all $x \in X, y \in Y$ and $\Phi \in N_{0}$. Assume $N_{0}>\mathcal{Z}\langle\Theta\rangle \simeq \Gamma \mathrm{L}\left(1, p^{n}\right)$. By [5,18] or [20] $N_{0} \simeq \Gamma \mathrm{L}\left(m, p^{b}\right)$, with $m b=n, m>1$. The representation of the operator $z_{a} \in \mathcal{Z}$ as $\operatorname{diag}\left(T_{0}(a), T_{0}\left(a^{-\bar{\ell}}\right), 1\right)$ leads to an obvious identification of $X$ and $Y$ with $F$. Let $S_{0} \simeq \operatorname{SL}\left(m, p^{b}\right) \unlhd N_{0}$. The groups $\left(S_{0}\right)_{X}$ and $\left(S_{0}\right)_{Y}$ (for both groups the normalizer in $\mathrm{GL}_{\mathbb{F}_{p}}(F)$ contains $T_{0}\left(F^{*}\right)$ ) have the same concrete description as a subgroup of $\operatorname{GL}_{\mathbb{F}_{p}}(F)$, which is given after the proof of Lemma 5.9. 
By Lemma $5.10 N_{\operatorname{Sym}(F)}\left(\left(S_{0}\right)_{Y}\right)=\left(N_{0}\right)_{Y}$. For $\Phi \in S_{0}$, define $\Phi_{X}^{*}$ as the unique operator in $\mathrm{GL}_{\mathbb{F}_{p}}(F)$, such that $\operatorname{Tr}\left(\left(x \Phi_{X}\right) y\right)=\operatorname{Tr}\left(x\left(y \Phi_{X}^{*}\right)\right)$ holds for all $x, y$ in $F$. Since $T_{0}\left(F^{*}\right)$ is a Singer group of $\mathrm{GL}_{\mathbb{F}_{p}}(F)$ contained in $\left(N_{0}\right)_{X} \cong\left(N_{0}\right)_{Y}$ and as $T_{0}\left(F^{*}\right)^{*}=T_{0}\left(F^{*}\right)$, we get $\Phi_{X}^{*} \in\left(S_{0}\right)_{X}^{*}=\left(S_{0}\right)_{Y}$ by Lemma 5.9. Let $\pi(\ell)$ be the permutation on $F$, which is induced by $x \mapsto x^{\ell}$. From $\mu(x, y)=\mu\left(x \Phi_{X}, y \Phi_{Y}\right)$, we deduce

$$
\operatorname{Tr}\left(x(y \pi(\ell))=\operatorname{Tr}\left(x \Phi_{X}\left(y \Phi_{Y} \pi(\ell)\right)\right)=\operatorname{Tr}\left(x\left(y \pi(\ell)\left(\pi(\ell)^{-1} \Phi_{Y} \pi(\ell) \Phi_{X}^{*}\right)\right)\right) .\right.
$$

This forces $y^{\prime}=y^{\prime} \pi(\ell)^{-1} \Phi_{Y} \pi(\ell) \Phi_{X}^{*}$ for all $y^{\prime} \in F$. So $1_{F}=\pi(\ell)^{-1} \Phi_{Y} \pi(\ell) \Phi_{X}^{*}$ or $\pi(\ell)^{-1} \Phi_{Y} \pi(\ell)=\left(\Phi_{X}^{*}\right)^{-1} \in\left(S_{0}\right)_{Y}$. Thus (we identify $F$ and $\left.Y\right) \pi(\ell) \in\left(N_{0}\right)_{Y}$, as we have seen above. But then $\pi(\ell)$ would be additive, a contradiction.

Proof (Of Corollary 5.2) Assume that $\mu=\mu_{\ell}$ and $\mu^{\prime}=\mu_{m}$ are EA equivalent. By Corollary 2.4, there exists $\Phi \in \operatorname{GL}(\mathcal{U}), \mathcal{U}=V \times \mathbb{F}_{p}$, fixing $V \times 0$ and $0 \times \mathbb{F}_{p}$, which sends the graph of $\mu$ onto the graph of $\mu^{\prime}$. In particular, $\mathbf{A}\left(\mu^{\prime}\right)=\Phi^{-1} \mathbf{A}(\mu) \Phi$. Our usual Frattini argument shows that we even may assume $\mathcal{Z}^{\prime}=\mathcal{Z}^{\Phi}$, where $\mathcal{Z}$ and $\mathcal{Z}^{\prime}$ are cyclic subgroups of $\mathbf{A}(\mu)$ and $\mathbf{A}\left(\mu^{\prime}\right)$, respectively, which correspond to the group $\mathcal{Z}$ of Example 3.13. Set $X=F \times 0 \times 0$ and $Y=0 \times F \times 0$. Then, $\mathcal{Z}_{W}=\mathcal{Z}_{W}^{\prime}=T_{0}\left(F^{*}\right)$, where we identify $W=X, Y$ in the obvious way with $F$.

Let $z$ be a generator of $\mathcal{Z}$. Then, $z^{\prime}=\Phi^{-1} z \Phi$ is a generator of $\mathcal{Z}^{\prime}$. If $\omega, \omega^{p}, \ldots, \omega^{p^{n-1}}$ are the eigenvalues of $z$ on $X(\omega$ a primitive element in $F)$, then $\omega^{-\bar{\ell}}, \omega^{-\bar{\ell} p}, \ldots, \omega^{-\bar{\ell} p^{n-1}}$ are the eigenvalues of $z$ on $Y$. Here, $\bar{\ell}$ is the inverse of $\ell$ modulo $p^{n}-1$. The same must hold for $z^{\prime}$, as these elements are conjugate by $\Phi$ and as this transformation fixes $X$ and $Y$. On the other hand, $z^{\prime}$ as an element of $\mathcal{Z}^{\prime}$ has on $Y$ the eigenvalues $\omega^{-\bar{m}}, \omega^{-\bar{m} p}, \ldots, \omega^{-\bar{m} p^{n-1}}$, where $\bar{m}$ is the inverse of $m$ modulo $p^{n}-1$. Hence, $-\bar{\ell} p^{k} \equiv-\bar{m}\left(\bmod p^{n}-1\right)$ for some $k$, i.e., $-\bar{\ell} \sim-\bar{m}$ and $\ell \sim m$ follows.

\section{Remarks to the case of even characteristic}

As already mentioned, the predecessor of this paper is [9]. The results of [9] look a bit different than the findings the present paper. We discuss how [9] and the present paper compare and point out the slight differences.

It is well known that a function $f: V=V(n, 2) \rightarrow \mathbb{F}_{2}$ is bent iff the counterimage of 0, i.e., $B(f)=f^{-1}(0)$ is a difference set in $V$. In [9], we investigated instead of bent functions rather their associated difference set $B(f)$ (called $B$-set there). As a result, $B$-sets $B\left(f_{1}\right)$ and $B\left(f_{2}\right)$ are equivalent in the sense of [9], iff $f_{1}$ and $f_{2}$ are affine equivalent. In other words, the results of [9] relate to affine equivalence of functions and are therefore slightly more special than the related results in the present paper. So extra work is needed to generalize the work of [9] to the level of the present paper. We do not expect any difficulty but refrained from this task to keep the size of the present paper limited. However, there is a quick transfer of Corollary 5.2 to the case of even characteristic, namely Theorem 6.1. 
The standard parabolic $B$-sets and the special parabolic $B$-sets are related to functions, which are exact analogs of standard parabolic and special parabolic bent functions in the present paper. However, the twisted parabolic $B$-sets correspond only

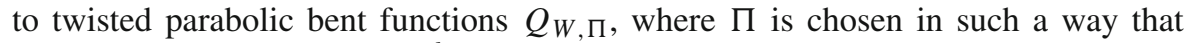
$C_{\mathrm{SO}(W / \operatorname{rad}(W))}(\Pi) \simeq \mathrm{SO}\left(a, p^{b}\right), m=a b$, where $\mathrm{SO}(W / \operatorname{rad}(W))=\mathrm{SO}(m, p)$.

The big $B$-sets of cyclic trace type in [9] associated with the automorphism $x \mapsto x^{k}$ of $\mathbb{F}_{2^{n}}^{*}$ in $V(2 n, 2)$ correspond to Maiorana functions $\mu_{\ell}$ in the present paper, where $k \ell \equiv 1\left(\bmod p^{n}-1\right)$. The study $B$-sets of cyclic trace type was elaborate as it required the investigation of irreducible subgroups of $\operatorname{GL}(2 n, 2)$, which contain semiregular cyclic subgroups of order $2^{n}-1$. The double derivative was allowed in the present paper to circumvent these obstacles. We note that double derivatives are useless in the even characteristic case, since they are trivial then. However, for certain numbers $\ell$ Gangopadhyay [13] is able to count the numbers of triples $v, w, x \in V(2 n, 2), v \neq w$ with $D_{v} D_{w} \mu_{\ell}(x)=0$. As a consequence, he obtains inequivalence results for some functions $\mu_{\ell}$.

Theorem 6.1 Let $n \geq 2, F=\mathbb{F}_{2^{n}}$ and $V=F \times F$, and let $0<\ell, m<2^{n}-1$ be coprime to $2^{n}-1$. Define bent functions $\mu_{\ell}, \mu_{m}: V \rightarrow \mathbb{F}_{2}$ by $\mu_{s}(x, y)=\operatorname{Tr}\left(x y^{s}\right)$ for $s=\ell$ or $=m$. Let $\mu_{\ell}$ and $\mu_{m}$ be EA equivalent. Then, $\ell \equiv m 2^{a}\left(\bmod 2^{n}-1\right)$ for some number $a$.

Proof Assume first that $n \neq 6$. Then, there exists a $p$-primitive (cf. [27]) prime divisor $r$ of $2^{n}-1$, i.e., $r$ divides $2^{n}-1$ but not $2^{k}-1$ for $1 \leq k<n$. Let $r^{a}$ be the largest prime power dividing $2^{n}-1$ and denote by $\mu_{s}$ either $\mu_{\ell}$ or $\mu_{m}$. The Sylow $r$-subgroup $\mathcal{Z}_{r}^{(s)}$ of $\mathcal{Z}^{(s)}$ (which plays the role of $\mathcal{Z}$ in Example 3.13) has order $r^{a}$. Let $R$ be a Sylow $r$ subgroup of EA $(\mu)$ containing $\mathcal{Z}_{r}^{(s)}$. We claim, $R \leq \operatorname{GL}(\mathcal{U}),(\mathcal{U}$ the ambient space of $\mu)$, and $[\mathcal{U}, R]=\langle u(\phi-1) \mid u \in \mathcal{U}, \phi \in R\rangle=F \times F \times 0$ and $C_{\mathcal{U}}(R)=0 \times 0 \times \mathbb{F}_{2}$ :

As $r$ is coprime to 2, the subgroup $\operatorname{GL}(\mathcal{U})$ contains Sylow $r$-subgroups of $\operatorname{AGL}(\mathcal{U})$. They are isomorphic to $\mathrm{C}_{r^{a}} \times \mathrm{C}_{r^{a}}$ (Clearly, $\mathrm{GL}(\mathcal{U})$ contains a subgroup $Z_{1} \times Z_{2} \simeq$ $\mathrm{C}_{2^{n}-1} \times \mathrm{C}_{2^{n}-1}$, where the groups $Z_{i}$ induce Singer groups on two disjoint $n$-subspaces $\mathcal{U}_{1}$ and $\mathcal{U}_{2}$. Note also that $r^{2 a}$ is the maximal $r$-power dividing the order of GL $(\mathcal{U})$.). In particular, Sylow $r$-subgroups are abelian. Let $\phi$ be in $R$. As $\phi$ centralizes $\mathcal{Z}_{r}$, this operator fixes $\left[\mathcal{U}, \mathcal{Z}_{r}^{(s)}\right]=F \times F \times 0$ and $C_{\mathcal{U}}\left(\mathcal{Z}_{r}^{(s)}\right)=0 \times 0 \times \mathbb{F}_{2}$. Moreover, one must have $\phi=\Phi \circ \tau_{u}$, with $u \in 0 \times 0 \times \mathbb{F}_{2}$. As $\phi$ has $r$-power order also $u=0$, forcing the claim.

Assume now that $\alpha \in \operatorname{AGL}(\mathcal{U})$ induces an EA isomorphism from $\mu_{\ell}$ to $\mu_{m}$. Let $R \leq \mathbf{A}\left(\mu_{\ell}\right) \cap \operatorname{GL}(\mathcal{U})$ be the Sylow $r$-subgroup containing $\mathcal{Z}_{r}^{(\ell)}$. By Sylow's theorem, we can assume $\mathcal{Z}_{r}^{(m)} \leq \alpha^{-1} R \alpha$. Then, $C_{\mathcal{U}}(R) \alpha=C_{\mathcal{U}}\left(\alpha^{-1} R \alpha\right)=C_{\mathcal{U}}(R)$ and $[\mathcal{U}, R] \alpha=\left[\mathcal{U}, \alpha^{-1} R \alpha\right]=[\mathcal{U}, R]$ by the claim above. So $\mu_{\ell}$ and $\mu_{m}$ are affine equivalent. By $[9, \mathrm{Thm} . \mathrm{B}]$, the assertion of the theorem follows.

For $n=6$, we observe: $\operatorname{dim} \mathcal{U}=13$, a Sylow 7-subgroup $R$ of GL $(\mathcal{U})$ is elementary abelian of order $7^{4}, \operatorname{dim}[R, \mathcal{U}]=12$ and $\operatorname{dim} C_{\mathcal{U}}(R)=1$. We can now repeat the arguments from above with 7 in the role of the prime $r$.

Acknowledgements The author thanks Peter Sin [23] for helpful discussions about the first cohomology of symplectic groups and especially for his proofs of Lemmas 2.18 and 2.19. I also thank Alexander Pott for bringing Reference [4] to my attention. Finally, I thank both referees their commentaries, which lead improvements of the presentation like Remark 3.2(a) and Theorem 6.1. 


\section{References}

1. Aschbacher, M.: Finite Group Theory. Cambridge University Press, Cambridge (2000)

2. Bell, G.: On the cohomology of the finite special linear groups, I. J. Algebra 54, 216-238 (1978)

3. Bosma, W., Cannon, J., Playoust, C.: The Magma algebra system I: the user language. J. Symbolic Comput. 24, 235-265 (1997)

4. Budaghyan, L., Carlet, C.: CCZ-equivalence of bent vectorial functions and related constructions. Des. Codes Cryptogr. 59, 69-87 (2011)

5. Cameron, P.: Primitive permutation groups and finite simple groups. Bull. Lond. Math. Soc. 13, 1-22 (1981)

6. Carter, R.: Simple Groups of Lie Type. Wiley, Hoboken (1989)

7. Cesmelioglu, A., Meidl, W., Pott, A.: Generalized Maiorana-McFarland class and the normality of p-ary bent functions. Finite Fields Appl. 24, 105-117 (2013)

8. Dempwolff, U.: A characterization of the generalized twisted field planes. Arch. Math. 50, 477-480 (1988)

9. Dempwolff, U.: Automorphisms and equivalence of bent functions and of difference sets in elementary abelian 2-groups. Comm. Algebra 34(3), 1077-1131 (2006)

10. Dempwolff, U.: Some doubly transitive bilinear dual hyperovals and their ambient spaces. European J. Combin. 44, 1-22 (2015)

11. Dempwolff, U.: The automorphism groups of doubly transitive bilinear dual hyperovals. Adv. Geom. 17, 91-108 (2017)

12. Dempwolff, U.: CCZ equivalence of power functions. Des. Codes Cryptogr. 86, 665-692 (2018)

13. Gangopadhyay, S.: Affine inequivalence of cubic Maiorana-McFarland type bent functions. Discrete Appl. Math. 161, 1141-1146 (2013)

14. Grove, L.: Classical Groups and Geometric Algebra. AMS Series Graduate Studies in Mathematics, vol. 39. Providence, RI (2002)

15. Harris, M.: A note on the classical groups over finite fields. Rev. Roumaine Math. Pures Appl. 27(2), 159-167 (1982)

16. Huppert, B.: Endliche Gruppen I. Die Grundlehren der Mathematischen Wissenschaften, vol. 134. Springer-Verlag, Berlin, New York (1967)

17. Jones, W., Parshall, B.: On the 1-cohomology of finite groups of Lie type. In: Proceedings of the Conference on Finite Groups (University of Utah, Park City, Utah, 1975), pp. 313-328. Academic Press, New York (1976)

18. Kantor, W.: Linear groups containing a Singer cycle. J. Algebra 62, 232-234 (1980)

19. Kumar, P., Scholz, R., Welch, L.: Generalized bent functions and their properties. J. Combin. Theory Ser. A 40, 90-107 (1985)

20. Liebeck, M.: The affine permutation groups of rank three. Proc. Lond. Math. Soc. (3) 54, 477-516 (1987)

21. Lübeck, F.: Small degree representations of finite Chevalley groups in defining characteristic. LMS J. Comput. Math. 4, 135-169 (2001)

22. Schneider, L.: Minimale Darstellungen endlicher klassischer Gruppen in natürlicher Charakteristik. Cuvillier Verlag, Göttingen (2004)

23. Sin, P.: Personal communication

24. Steinberg, R.: Lectures on Chevalley Groups. AMS-University Lecture Series, vol. 66. Providence, RI (2016)

25. The GAP Group: GAP-Groups Algorithms and Programming, version 4.4.12 (2008). http://www.gapsystem.org. Accessed 15 Jan 2018

26. Völklein, F.: The 1-Cohomology of the adjoint module of a Chevalley group. Forum Math. 1, 1-13 (1989)

27. Zsigmondy, K.: Zur Theorie der Potenzreste. Monatshefte Math. Phys. 3(3), 265-284 (1892)

Publisher's Note Springer Nature remains neutral with regard to jurisdictional claims in published maps and institutional affiliations. 